\title{
Non-adiabatic Matsubara Dynamics and Non-adiabatic Ring Polymer Molecular Dynamics
}

\author{
Sutirtha N. Chowdhury ${ }^{\text {a) }}$ and Pengfei Huo ${ }^{\text {b) }}$ \\ Department of Chemistry, University of Rochester, 120 Trustee Road, Rochester, New York 14627, \\ United States and \\ The Institute of Optics, University of Rochester, Rochester, New York, 14627
}

\begin{abstract}
We present the non-adiabatic Matsubara dynamics, a general framework for computing the time-correlation function (TCF) of electronically non-adiabatic systems. This new formalism is derived based on the generalized Kubo-transformed time-correlation function, using the Wigner representation for both the nuclear degrees of freedom (DOF) and the electronic mapping variables. By dropping the non-Matsubara nuclear normal modes in the quantum Liouvillian and explicitly integrate these modes out of the TCF, we derived the non-adiabatic Matsubara dynamics approach. Further making the approximation to drop the imaginary part of the Matsubara Liouvillian and enforce the nuclear momentum integral to be real, we arrived at the non-adiabatic ring-polymer molecular dynamics (NRPMD) approach. We have further justified the capability of NRPMD for simulating the non-equilibrium time-correlation function. This work provides the rigorous theoretical foundation for several recently proposed state-dependent RPMD approaches and offers a general framework for developing new non-adiabatic quantum dynamics approaches in the future.
\end{abstract}

\section{INTRODUCTION}

Accurately simulating the quantum dynamics of the molecular system remains a central challenge in theoretical chemistry, due to the difficulties of accurately describing electronically non-adiabatic dynamics and nuclear quantum dynamics. Directly performing exact quantum dynamics simulations is computationally demanding, despite exciting recent progress. ${ }^{1-11}$

To accurately describe the non-adiabatic dynamics, a large number of these approaches are developed, including the popular trajectory surface-hopping method (mixed quantum-classical approach),${ }^{12-15}$ the linearized path-integral approaches, ${ }^{16-23}$ and the mixed quantumclassical Liouville equation, ${ }^{24-28}$ Despite providing accurate electronic non-adiabatic dynamics, these approaches often relies on the Wigner sampling of the initial nuclear distribution and a classical dynamics for propagation. Thus in generally, they do not preserve quantum Boltzmann distribution $(\mathrm{QBD})^{29,30}$ or zero point energy (ZPE) associated with the nuclear degrees of freedom (DOF). They often suffer from numerical issues such as ZPE leakage, ${ }^{31,32}$ although significant improvements are accomplished through the recent development. ${ }^{33-36}$

To accurately describe nuclear quantum dynamics for electronically adiabatic systems, imaginary-time path integral based approaches ${ }^{37-39}$ are developed, including the centroid molecular dynamics (CMD) ${ }^{40-42}$ and the ringpolymer molecular dynamics (RPMD). ${ }^{43,44}$ In particular, RPMD resembles the classical MD in the extended phase space, thus provides a convenient way to compute approximate quantum time-correlation functions. ${ }^{43}$ The classical evolution of RPMD preserves its initial quan-

\footnotetext{
a) Electronic mail: schowdh4@ur.rochester.edu

b)Electronic mail: pengfei.huo@rochester.edu
}

tum distribution captured by the ring-polymer Hamiltonian, and it is free of the zero-point energy leaking problem. ${ }^{31,43}$ Despite its success on describing quantum effects in the condensed phase, RPMD is limited to one-electron non-adiabatic dynamics ${ }^{45-49}$ or nuclear quantization, ${ }^{43,50-53}$ as well as the lack of real-time electronic coherence effects. ${ }^{45,46}$

Recently emerged state-dependent RPMD approaches provide a unified description of both the electronically non-adiabatic dynamics and nuclear quantum effects. These state-dependent RPMD methods, such as non-adiabatic RPMD (NRPMD), ${ }^{54-56}$ mapping variable RPMD (MV-RPMD), ${ }^{57-59}$ ring-polymer Ehrenfest dynamics, ${ }^{60}$, kinetically-constrained RPMD (KCRPMD), ${ }^{48,61,62}$ coherent state RPMD (CS-RPMD), ${ }^{63}$ and ring-polymer surface hopping (RPSH) ${ }^{64-68}$ are promising to provide both accurate non-adiabatic dynamics with an explicit description of electronic states, as well as a reliable treatment of nuclear quantum dynamics through the ring polymer path-integral quantization.

Despite the initial success, all of the above statedependent RPMD approaches are currently viewed as the model dynamics. The Hamiltonians associated with some these approaches (such as MV-RPMD) are derived from quantum partition function, and these Hamiltonians are directly used for dynamics propagation as well as the initial sampling. Thus the fundamental and crucial theoretical question is that can these methods be rigorously justified. If so, not only it will explain the numerical success of these state-dependent RPMD approaches, but also it will offer a general theoretical framework to understand the limitations of these state-dependent RPMD approaches and further improving them. Recent theoretical work on the Matsubara dynamics ${ }^{69-72}$ by Althrope and co-workers indeed provides hope for this, because RPMD (as well as CMD) can be derived as an approximation of the Matsubara dynamics, ${ }^{70,73}$ which itself can be rigorously derived. ${ }^{69}$ 
In this paper, we present the non-adiabatic Matsubara dynamics, a general framework for computing the time-correlation function of electronically non-adiabatic systems. This new formalism is derived based on the generalized Kubo-transformed time-correlation function (TCF) formalism ${ }^{42,69}$, using the Wigner representation for both the nuclear DOF and electronic mapping variables. ${ }^{74-76}$ By dropping the non-Matsubara nuclear normal modes in the quantum Liouvillian, ${ }^{69}$ we derived the non-adiabatic Matsubara dynamics, which can be viewed as a generalization of the original (electronically adiabatic) Matsubara dynamics. ${ }^{69}$ Further making the approximation that drop the imaginary part of the Matsubara Liouvillian, ${ }^{73}$ we arrived at the non-adiabatic RPMD (NRPMD) approach, where the initial distribution coincides with the one in Mapping-Variable (MV)$\mathrm{RPMD}^{57}$, whereas the Liouvillian coincides with the Liouvillian used in the originally proposed NRPMD ${ }^{54}$. We have further justified the capability of NRPMD for simulating the non-equilibrium time correlation function.

\section{GENERALIZED KUBO-TRANSFORMED TIME-CORRELATION FUNCTION}

We begin by introducing the Generalized Kubo Transformed time-correlation functions for a state-dependent Hamiltonian. We start by expressing the total Hamiltonian operator as follows

$$
\hat{H}=\hat{T}+\hat{V}_{0}+\hat{V}_{e}=\frac{\hat{P}^{2}}{2 m}+V_{0}(\hat{R})+\sum_{i, j=1}^{\mathcal{K}} \mathcal{V}_{i j}(\hat{R})|i\rangle\langle j|
$$

where $\{|i\rangle\}$ is the diabatic basis, $\hat{T}$ is the nuclear kinetic energy operator, $\hat{R}$ is the nuclear position operator with the corresponding conjugate momentum operator $\hat{P}$. To simplify our discussion, we have assumed that there is only one nuclear DOF in the system. Generalizing the discussion with many nuclear DOF is straightforward.

Further, $V_{0}(\hat{R})$ is the state-independent potential operator, whereas $\hat{V}_{\mathrm{e}}=\sum_{i j} \mathcal{V}_{i j}(\hat{R})|i\rangle\langle j| \equiv \mathcal{V}(R)$ is the state-dependent potential operator (electronic part of the Hamiltonian) with $\mathcal{K}$ total diabatic electronic states. We assume that $\mathcal{V}_{i j}(\hat{R})$ is real and symmetric through out this work.

\section{A. Mapping Representation of Electronic States}

In the electronic part of the Hamiltonian, the $\mathcal{K}$ diabatic electronic states can be mapped into $\mathcal{K}$ harmonic oscillators' ground and excited states, where $(\mathcal{K}-1)$ oscillator in ground state and $i_{t h}$ oscillator in the first excited state. It can be formally written as

$$
|i\rangle \rightarrow\left|0_{1}, \ldots, 1_{i} \ldots, 0_{\mathcal{K}}\right\rangle=\hat{a}_{i}^{\dagger}\left|0_{1}, \ldots, 0_{i} \ldots, 0_{\mathcal{K}},\right\rangle
$$

where $\hat{a}_{i}^{\dagger}=\frac{1}{\sqrt{2 \hbar}}\left(\hat{q}_{i}-i \hat{p}_{i}\right)$ and $\hat{a}_{j}=\frac{1}{\sqrt{2 \hbar}}\left(\hat{q}_{j}+i \hat{p}_{j}\right)$, and $\hat{\mathbf{q}}=\left\{\hat{q}_{1}, \ldots \hat{q}_{i}, \ldots \hat{q}_{\mathcal{K}}\right\}$ and $\hat{\mathbf{p}}=\left\{\hat{p}_{1}, \ldots \hat{p}_{i}, \ldots \hat{p}_{\mathcal{K}}\right\}$ are the mapping position and momentum operator respectively. This mapping formalism is often referred to as the Meyer-Miller-Stock-Thoss (MMST) ${ }^{74-76}$ mapping representation. With this mapping formalism, the electronic part of the Hamiltonian is expressed as

$$
\sum_{i, j=1}^{\mathcal{K}} \mathcal{V}_{i j}(\hat{R})|i\rangle\langle j| \rightarrow \sum_{i, j=1}^{\mathcal{K}} \mathcal{V}_{i j}(\hat{R}) \hat{a}_{i}^{\dagger} \hat{a}_{j}
$$

Using the above relationships, the electronic part of the Hamiltonian in Eq. 1 can be represented as

$$
\hat{V}_{\mathrm{e}}=\frac{1}{2 \hbar} \sum_{i, j=1}^{\mathcal{K}} \mathcal{V}_{i j}(\hat{R})\left(\hat{q}_{i} \hat{q}_{j}+\hat{p}_{i} \hat{p}_{j}-\delta_{i j} \hbar\right) .
$$

This is known as the MMST ${ }^{74-76}$ mapping Hamiltonian.

\section{B. Generalized Kubo-Transformed Time-Correlation Functions}

We begin by writing the generalized Kubo-transformed time-correlation function (TCF). The conventional Kubo transformed correlation function is defined as

$$
\begin{aligned}
C_{A B}^{\mathrm{K}}(t) & =\frac{1}{\mathcal{Z} \beta} \int_{0}^{\beta} d \lambda \operatorname{Tr}\left[e^{-(\beta-\lambda) \hat{H}} \hat{A} e^{-\lambda \hat{H}} e^{\frac{i}{\hbar} \hat{H} t} \hat{B} e^{-\frac{i}{\hbar} \hat{H} t}\right] \\
& =\frac{1}{\mathcal{Z} N} \sum_{k=1}^{N} \operatorname{Tr}\left[e^{-\beta_{N}(N-k) \hat{H}} \hat{A} e^{-\beta_{N} k \hat{H}} e^{\frac{i}{\hbar} \hat{H} t} \hat{B} e^{-\frac{i}{\hbar} \hat{H} t}\right]
\end{aligned}
$$

where $\beta=1 / k_{\mathrm{B}} T$ is the inverse temperature, $\mathcal{Z}=$ $\operatorname{Tr}\left[e^{-\beta \hat{H}}\right]$ is the canonical partition function, $\hat{H}$ is defined in Eq. 1, and $\operatorname{Tr}=\operatorname{Tr}_{\mathrm{n}} \operatorname{Tr}_{\mathrm{e}}$ represents the trace over both electronic and nuclear DOFs. From the first to the second expression, we have converted a definite integral into the discrete Riemann sum through $\int_{a}^{b} f(\lambda) d \lambda=$ $\lim _{N \rightarrow \infty} \sum_{k=1}^{N} f(a+k \cdot \Delta \lambda) \cdot \Delta \lambda$, where $a=0, b=\beta$, and $\Delta \lambda=\beta / N=\beta_{N}$. Note that the second line of Eq. 5 is equivalent to the first line under the limit of $N \rightarrow \infty$.

We further insert $N-1$ identities of the form $e^{i \hat{H} t / \hbar} e^{-i \hat{H} t / \hbar}=\hat{\mathbb{1}}$ in Eq. 5 (see details in the Supporting Information), resulting in

$$
\begin{aligned}
C_{A B}^{[N]}(t) & =\frac{1}{\mathcal{Z}} \frac{1}{N} \sum_{k=1}^{N} \operatorname{Tr}\left[\left(e^{-\beta_{N} \hat{H}} e^{\frac{i}{\hbar} \hat{H} t} e^{-\frac{i}{\hbar} \hat{H} t}\right)^{N-k-1}\right. \\
& \times e^{-\beta_{N} \hat{H}} \hat{A} e^{i \hat{H} t / \hbar} e^{-i \hat{H} t / \hbar}\left(e^{-\beta_{N} \hat{H}} e^{i \hat{H} t / \hbar} e^{-i \hat{H} t / \hbar}\right)^{k-1} \\
& \left.\times e^{-\beta_{N} \hat{H}} e^{i \hat{H} t / \hbar} \hat{B} e^{-i \hat{H} t / \hbar}\right]
\end{aligned}
$$

where $\beta_{N}=\beta / N$. Note that Eq. 6 has a symmetric block structure of the form $e^{-\beta_{N} \hat{H}} e^{i \hat{H} t / \hbar} e^{-i \hat{H} t / \hbar}$, where the operator $\hat{A}$ is evaluated inside a particular block depending on the value of the $k$ index. This type of generalized 
Kubo-Transformed time-correlation function was first introduced in the work to connect Linearized Path-integral approach and CMD, ${ }^{42}$ and later used for the development of the Matsubara dynamics. ${ }^{69-72}$

A path integral representation of Eq. 6 can be obtained by inserting the following identities

$$
\begin{aligned}
\hat{\mathbb{1}}_{R_{l}^{\prime}, \mathbf{q}_{l}^{\prime}} & =\int d R_{l}^{\prime} \int d \mathbf{q}_{l}^{\prime} \hat{\mathcal{P}}\left|R_{l}^{\prime}, \mathbf{q}_{l}^{\prime}\right\rangle\left\langle R_{l}^{\prime}, \mathbf{q}_{l}^{\prime}\right| \\
\hat{\mathbb{1}}_{R_{l}^{\prime \prime}, \mathbf{q}_{l}^{\prime \prime}} & =\int d R_{l}^{\prime \prime} \int d \mathbf{q}_{l}^{\prime \prime}\left|R_{l}^{\prime \prime}, \mathbf{q}_{l}^{\prime \prime}\right\rangle\left\langle R_{l}^{\prime \prime}, \mathbf{q}_{l}^{\prime \prime}\right| \hat{\mathcal{P}}
\end{aligned}
$$

where the bead index $l=1 \ldots N,\left\{R_{l}^{\prime \prime}, R_{l}^{\prime}\right\}$ and $\left\{\mathbf{q}_{l}^{\prime \prime}, \mathbf{q}_{l}^{\prime}\right\}$ are the nuclear and mapping variable position, respectively, with $\mathbf{q}_{l}^{\prime}=\left\{\left[q_{l}^{\prime}\right]_{1}, \ldots\left[q_{l}^{\prime}\right]_{i}, \ldots\left[q_{l}^{\prime}\right]_{\mathcal{K}}\right\}$ and similarly for $\mathbf{q}_{l}^{\prime \prime}$ for the corresponding momenta. Further, the electronic projection operator is

$$
\hat{\mathcal{P}}=\sum_{i=1}^{\mathcal{K}}|i\rangle\langle i|
$$

which helps to confine the mapping DOFs within the correct SEO subspace. $^{77}$

Inserting Eq. 7-8 into the bead-specific positions of Eq. 6, followed by replacing the full trace over the nuclear and electronic DOFs with the phase space integrals, ${ }^{78}$ one arrives at the formal mathematical description of Generalized Kubo correlation function ${ }^{69,78}$ as follows

$$
\begin{aligned}
C_{A B}^{[N]}(t)=\frac{1}{\mathcal{Z}} & \int d \mathbf{R}^{\prime \prime} \int d \mathbf{R}^{\prime} \int d \mathbf{q}^{\prime} \int d \mathbf{q}^{\prime \prime} \\
& \times \frac{1}{N} \sum_{k=1}^{N} \prod_{l \neq k}^{N}\left\langle R_{l-1}^{\prime \prime}, \mathbf{q}_{l-1}^{\prime \prime}\left|\hat{\mathcal{P}} e^{-\beta_{N} \hat{H}} \hat{\mathcal{P}}\right| R_{l}^{\prime}, \mathbf{q}_{l}^{\prime}\right\rangle \\
& \times\left\langle R_{k-1}^{\prime \prime}, \mathbf{q}_{k-1}^{\prime \prime}\left|\hat{\mathcal{P}} e^{-\beta_{N} \hat{H}} \hat{\mathcal{P}} \hat{A}\right| R_{k}^{\prime}, \mathbf{q}_{k}^{\prime}\right\rangle \\
& \times \frac{1}{N} \sum_{k^{\prime}=1}^{N} \prod_{l \neq k^{\prime}}^{N}\left\langle R_{l}^{\prime}, \mathbf{q}_{l}^{\prime}\left|e^{\frac{i}{\hbar} \hat{H} t} e^{-\frac{i}{\hbar} \hat{H} t}\right| R_{l}^{\prime \prime}, \mathbf{q}_{l}^{\prime \prime}\right\rangle \\
& \times\left\langle R_{k^{\prime}}^{\prime}, \mathbf{q}_{k^{\prime}}^{\prime}\left|e^{\frac{i}{\hbar} \hat{H} t} \hat{B} e^{-\frac{i}{\hbar} \hat{H} t}\right| R_{k^{\prime}}^{\prime \prime}, \mathbf{q}_{k^{\prime}}^{\prime \prime}\right\rangle
\end{aligned}
$$

where we introduce the notation $\int d \mathbf{x}=\int \prod_{l=1}^{N} d \mathrm{x}_{l}$ for $\mathbf{x}=\left\{\mathbf{R}^{\prime}, \mathbf{R}^{\prime \prime}, \mathbf{q}^{\prime}, \mathbf{q}^{\prime \prime}\right\}$, and we have used the cyclicsymmetric property and write operator $\hat{B}$ also into a bead-averaged fashion. A detail derivation of Eq. 10 is provided in the supporting information.

To proceed, we change the variables $\left(R_{l}^{\prime}, R_{l}^{\prime \prime}, \mathbf{q}_{l}^{\prime}, \mathbf{q}_{l}^{\prime \prime}\right)$ into the mean $\left(R_{l}\right.$ and $\left.\mathbf{q}_{l}\right)$ and difference coordinates $\left(D_{l}\right.$ and $\left.\boldsymbol{\Delta}_{l}\right)$ as follows ${ }^{42,78}$

$$
\begin{aligned}
& R_{l}=\frac{1}{2}\left(R_{l}^{\prime}+R_{l}^{\prime \prime}\right), \quad \mathbf{q}_{l}=\frac{1}{2}\left(\mathbf{q}_{l}^{\prime}+\mathbf{q}_{l}^{\prime \prime}\right) \\
& D_{l}=R_{l}^{\prime}-R_{l}^{\prime \prime}, \quad \boldsymbol{\Delta}_{l}=\mathbf{q}_{l}^{\prime}-\mathbf{q}_{l}^{\prime \prime} .
\end{aligned}
$$

Noting that the Jacobian of the above transformation is unity for each bead index $l$. With this transformation, one can re-express the Eq. 10 as

$$
\begin{aligned}
C_{A B}^{[N]}(t)=\frac{1}{\mathcal{Z}} & \int d \mathbf{R} \int d \mathbf{D} \int d \mathbf{q} \int d \boldsymbol{\Delta} \\
& \times \frac{1}{N} \sum_{k=1}^{N} \prod_{l \neq k}^{N}\left\langle\mathbf{q}_{l-1}-\frac{1}{2} \boldsymbol{\Delta}_{l-1}, R_{l-1}-\frac{1}{2} D_{l-1}\right| \\
& \times \hat{\mathcal{P}} e^{-\beta_{N} \hat{H}} \hat{\mathcal{P}}\left|\mathbf{q}_{l}+\frac{\boldsymbol{\Delta}_{l}}{2}, R_{l}+\frac{D_{l}}{2}\right\rangle \\
& \times\left\langle\mathbf{q}_{k-1}-\frac{1}{2} \boldsymbol{\Delta}_{k-1}, R_{k-1}-\frac{1}{2} D_{k-1}\right| \\
& \times \hat{\mathcal{P}} e^{-\beta_{N} \hat{H}} \hat{\mathcal{P}} \hat{A}\left|q_{k}+\frac{\boldsymbol{\Delta}_{k}}{2}, R_{k}+\frac{D_{k}}{2}\right\rangle \\
& \times \frac{1}{N} \sum_{k^{\prime}=1}^{N} \prod_{l \neq k^{\prime}}^{N}\left\langle\mathbf{q}_{l}-\frac{\boldsymbol{\Delta}_{l}}{2}, R_{l}-\frac{D_{l}}{2}\right| e^{\frac{i}{\hbar} \hat{H} t} \\
& \times e^{-\frac{i}{\hbar} \hat{H} t}\left|\mathbf{q}_{l}+\frac{\boldsymbol{\Delta}_{l}}{2}, R_{l}+\frac{D_{l}}{2}\right\rangle \\
& \times\left\langle\mathbf{q}_{k^{\prime}}-\frac{\boldsymbol{\Delta}_{k^{\prime}}}{2}, R_{k^{\prime}}-\frac{D_{k^{\prime}}}{2}\right| e^{\frac{i}{\hbar} \hat{H} t} \hat{B} e^{-\frac{i}{\hbar} \hat{H} t} \\
& \times\left|\mathbf{q}_{k^{\prime}}+\frac{\boldsymbol{\Delta}_{k^{\prime}}}{2}, R_{k^{\prime}}+\frac{D_{k^{\prime}}}{2}\right\rangle .
\end{aligned}
$$

Next, inserting the following identities

$1=\int d D_{l}^{\prime} \delta\left(D_{l}+D_{l}^{\prime}\right)=\frac{1}{(2 \pi \hbar)} \int d D_{l}^{\prime} \int d P_{l} e^{\frac{i}{\hbar} P_{l}\left(D_{l}+D_{l}^{\prime}\right)}$

$1=\int d \boldsymbol{\Delta}_{l}^{\prime} \delta\left(\boldsymbol{\Delta}_{l}+\boldsymbol{\Delta}_{l}^{\prime}\right)=\frac{1}{(2 \pi \hbar)^{\mathcal{K}}} \int d \boldsymbol{\Delta}_{l}^{\prime} \int d \mathbf{p}_{l} e^{\frac{i}{\hbar} \mathbf{p}_{l} \cdot\left(\boldsymbol{\Delta}_{l}+\boldsymbol{\Delta}_{l}^{\prime}\right)}$

into Eq. 13 for all $l$ blocks, we have

$C_{A B}^{[N]}(t)=\frac{\alpha_{N}}{\mathcal{Z}} \int \mathrm{d} \mathbf{R} \int \mathrm{d} \mathbf{P} \int \mathrm{d} \mathbf{q} \int \mathrm{d} \mathbf{p}\left[e^{-\beta \hat{H}} \hat{A}\right]_{\bar{N}}[\hat{B}(t)]_{N}$,

with the constant $\alpha_{N}=1 /(2 \pi \hbar)^{(k+1) N}$, and the operator $\left[e^{-\beta \hat{H}} \hat{A}\right]_{\bar{N}}$ is expressed as

$$
\begin{aligned}
& {\left[e^{-\beta \hat{H}} \hat{A}\right]_{\bar{N}}=\frac{1}{N} \sum_{k=1}^{N} \int d \mathbf{D} \int d \boldsymbol{\Delta} \prod_{l=1}^{N} e^{\frac{i}{\hbar} P_{l} D_{l}} e^{\frac{i}{\hbar} \mathbf{p}_{l} \boldsymbol{\Delta}_{l}}} \\
& \times \prod_{l \neq k}^{N}\left\langle\mathbf{q}_{l-1}-\frac{1}{2} \boldsymbol{\Delta}_{l-1}, R_{l-1}-\frac{1}{2} D_{l-1}\right| \\
& \hat{\mathcal{P}} e^{-\beta_{N} \hat{H}} \hat{\mathcal{P}}\left|\mathbf{q}_{l}+\frac{\boldsymbol{\Delta}_{l}}{2}, R_{l}+\frac{D_{l}}{2}\right\rangle \\
& \times\left\langle\mathbf{q}_{k-1}-\frac{1}{2} \boldsymbol{\Delta}_{k-1}, R_{k-1}-\frac{1}{2} D_{k-1}\right| \\
& \times \hat{\mathcal{P}} e^{-\beta_{N} \hat{H}} \hat{\mathcal{P}} \hat{A}\left|\mathbf{q}_{k}+\frac{\boldsymbol{\Delta}_{k}}{2}, R_{k}+\frac{D_{k}}{2}\right\rangle,
\end{aligned}
$$

and $[\hat{B}(t)]_{N}$ is expressed as follows

$$
\begin{aligned}
& {[\hat{B}(t)]_{N}=\frac{1}{N} \sum_{k=1}^{N} \int d \mathbf{D}^{\prime} \int d \boldsymbol{\Delta}^{\prime} \prod_{l=1}^{N} e^{\frac{i}{\hbar} P_{l} D_{l}^{\prime}} e^{\frac{i}{\hbar} \mathbf{p}_{l} \boldsymbol{\Delta}_{l}^{\prime}}} \\
& \times \prod_{l \neq k}^{N}\left\langle\mathbf{q}_{l}-\frac{1}{2} \boldsymbol{\Delta}_{l}^{\prime}, R_{l}-\frac{1}{2} D_{l}^{\prime}\left|e^{\frac{i}{\hbar} \hat{H} t} e^{-\frac{i}{\hbar} \hat{H} t}\right| \mathbf{q}_{l}+\frac{1}{2} \boldsymbol{\Delta}_{l}^{\prime}, R_{l}+\frac{1}{2} D_{l}^{\prime}\right\rangle \\
& \times\left\langle\mathbf{q}_{k}-\frac{1}{2} \boldsymbol{\Delta}_{k}^{\prime}, R_{k}-\frac{1}{2} D_{k}^{\prime}\left|e^{\frac{i}{\hbar} \hat{H} t} \hat{B} e^{-\frac{i}{\hbar} \hat{H} t}\right| \mathbf{q}_{k}+\frac{1}{2} \boldsymbol{\Delta}_{k}^{\prime}, R_{k}+\frac{1}{2} D_{k}^{\prime}\right\rangle
\end{aligned}
$$


where we have changed the dummy variable from $\sum_{k^{\prime}}$ to $\sum_{k}$. Integrals over $\mathbf{R}, \mathbf{P}, \mathbf{D}$ are $N$ dimensional, whereas integrals over $\mathbf{q}, \mathbf{p}, \boldsymbol{\Delta}$ are $(N \times \mathcal{K})$ dimensional. Note that $\left[e^{-\beta \hat{H}} \hat{A}\right]_{\bar{N}}$ in Eq. 15 contains a complex structure of the Wigner transform which couples with adjacent beads $l$ and $l+1$, whereas $[\hat{B}(t)]_{N}$ in Eq. 16 is a simple bead average of the Wigner transform.

We want to remind the reader that $C_{A B}^{[N]}(t)$ in Eq. 14 should be viewed as a generalized Kubo-Transformed time-correlation function, such that under the $N \rightarrow \infty$ limit, it returns to the original definition of the Kubotransformed time correlation function $C_{A B}^{\mathrm{K}}(t)$ defined in Eq. 5. With a finite $N$, even though it is no longer equivalent to $C_{A B}^{\mathrm{K}}(t)$, it is still an quantum mechanically exact time-correlation function.

\section{The Quantum Liouvillian}

For a Wigner transform of an operator $[\hat{A}]_{\mathrm{W}}=$ $\int d D e^{\frac{i}{\hbar} P D}\left\langle R-\frac{D}{2}|\hat{A}| R+\frac{D}{2}\right\rangle$, one can formally write down its time-dependent average as $[\hat{A}(t)]_{\mathrm{W}}=$ $\left[e^{\frac{i}{\hbar} \hat{H} t} \hat{A} e^{-\frac{i}{\hbar} \hat{H} t}\right]_{\mathrm{W}}=e^{i \mathcal{L} t}[\hat{A}(0)]_{\mathrm{W}}$, where $\mathcal{L}$ is the quantum Liouvillian (see Appendix A for detail derivation), which is also commonly referred to as the Wigner-Moyal series. ${ }^{79,80}$

Note that $[\hat{B}(t)]_{N}$ (Eq. 16) is expressed as the beadaveraged $(\mathcal{K}+1)$-dimensional Wigner transform as follows

$$
\begin{aligned}
& {[\hat{B}(t)]_{N}=\frac{1}{N} \sum_{k} \int d D_{k} \int d \boldsymbol{\Delta}_{k} e^{\frac{i}{\hbar} P_{k} D_{k}} e^{\frac{i}{\hbar} \mathbf{p}_{k} \boldsymbol{\Delta}_{k}}} \\
& \quad \times\left\langle\mathbf{q}_{k}-\frac{1}{2} \boldsymbol{\Delta}_{k}, R_{k}-\frac{1}{2} D_{k}|\hat{B}(t)| \mathbf{q}_{k}+\frac{1}{2} \boldsymbol{\Delta}_{k}, R_{k}+\frac{1}{2} D_{k}\right\rangle \\
& \equiv \frac{1}{N} \sum_{k}\left[\hat{B}_{k}(t)\right]_{\mathrm{W}},
\end{aligned}
$$

where $\hat{B}(t)=e^{\frac{i}{\hbar} \hat{H} t} \hat{B} e^{-\frac{i}{\hbar} \hat{H} t}$ and $\left[\hat{B}_{k}(t)\right]_{\mathrm{w}}$ is the Wigner transform of $\hat{B}(t)$ associated with the $k_{\text {th }}$ bead, defined in the above equation. In Eq. 17 we have also changed the dummy variables from $D^{\prime}$ and $\Delta^{\prime}$ to $D$ and $\Delta$. When $t=0,[\hat{B}(0)]_{N}=\frac{1}{N} \sum_{k}\left[\hat{B}_{k}\right]_{\mathrm{W}}$, with $\left[\hat{B}_{k}\right]_{\mathrm{W}}=\int d D_{k} \int d \boldsymbol{\Delta}_{k} e^{\frac{i}{\hbar} P_{k} D_{k}} e^{\frac{i}{\hbar} \mathbf{p}_{k} \boldsymbol{\Delta}_{k}}\left\langle\mathbf{q}_{k}-\frac{1}{2} \boldsymbol{\Delta}_{k}, R_{k}-\right.$ $\left.\frac{1}{2} D_{k}|\hat{B}| \mathbf{q}_{k}+\frac{1}{2} \boldsymbol{\Delta}_{k}, R_{k}+\frac{1}{2} D_{k}\right\rangle$.

With the above information, we can formally write $[\hat{B}(t)]_{N}$ as follows

$$
[\hat{B}(t)]_{N}=e^{i \mathcal{L}^{[N]} t}[\hat{B}(0)]_{N} .
$$

The Quantum Liouvillian $\mathcal{L}^{[N]}$ has the following form

$$
\mathcal{L}^{[N]}=\sum_{l} \frac{2}{\hbar}\left[\hat{H}_{l}\right]_{\mathrm{w}} \cdot \sin \left(\frac{\hbar}{2} \hat{\Lambda}_{l}\right)
$$

where $\hat{\Lambda}_{l}$ is the negative Poisson operator associated with the $l_{\text {th }}$ bead expressed as

$$
\hat{\Lambda}_{l}=\left(\begin{array}{c}
\hat{\Lambda}_{l}^{\mathrm{n}} \\
\hat{\Lambda}_{l}^{\mathrm{e}}
\end{array}\right)=\left(\begin{array}{c}
\frac{\overleftarrow{\partial}}{\partial P_{l}} \frac{\vec{\partial}}{\partial R_{l}}-\frac{\overleftarrow{\partial}}{\partial R_{l}} \frac{\vec{\partial}}{\partial P_{l}} \\
\overleftarrow{\nabla}_{\mathbf{p}_{l}} \vec{\nabla}_{\mathbf{q}_{l}}-\overleftarrow{\nabla}_{\mathbf{q}_{l}} \vec{\nabla}_{\mathbf{p}_{l}}
\end{array}\right)
$$

with the mapping variables related derivatives defined as

$$
\nabla_{\mathbf{q}}=\left(\begin{array}{c}
\frac{\partial}{\partial q_{1}} \\
\vdots \\
\frac{\partial}{\partial q_{\mathcal{K}}}
\end{array}\right)
$$

and $\nabla_{\mathbf{q}}^{T}=\left(\frac{\partial}{\partial q_{1}}, \ldots \frac{\partial}{\partial q_{\mathcal{K}}}\right)$, and likewise for $\boldsymbol{\nabla}_{\mathbf{p}}$.

Further, $\left[\hat{H}_{l}\right]_{\mathrm{W}}$ in Eq. 19 is the Wigner transform of MMST mapping Hamiltonian (Eq. 1) associated with the $l_{\text {th }}$ bead, which can be shown ${ }^{25,56,81}$ as

$$
\left[\hat{H}_{l}\right]_{\mathrm{W}}=\frac{P_{l}^{2}}{2 m}+V_{0}\left(R_{l}\right)+V_{\mathrm{e}}\left(R_{l}, \mathbf{q}_{l}, \mathbf{p}_{l}\right) .
$$

The detailed the proof of the above Wigner transform is provided in the Supporting Information. In the above equation, $V_{0}\left(R_{l}\right)$ is the state-independent potential evaluated at the $l_{\text {th }}$ nuclear bead position $R_{l}$, and $V_{\mathrm{e}}\left(\mathrm{R}_{l}, \mathrm{q}_{l}, \mathrm{p}_{l}\right)$ is the state dependent potential that parametrically depends on $R_{l}, \mathbf{q}_{l}=\left(\left[q_{l}\right]_{1},\left[q_{l}\right]_{2}, \ldots\left[q_{l}\right]_{\mathcal{K}}\right)$, and $\mathbf{p}_{l}=\left(\left[p_{l}\right]_{1},\left[p_{l}\right]_{2}, \ldots\left[p_{l}\right]_{\mathcal{K}}\right)$, with the following expression

$V_{\mathrm{e}}\left(\mathrm{R}_{l}, \mathbf{q}_{l}, \mathbf{p}_{l}\right)=\frac{1}{2 \hbar} \sum_{i, j=1}^{\mathcal{K}} \mathcal{V}_{i j}\left(R_{l}\right)\left(\left[p_{l}\right]_{i}\left[p_{l}\right]_{j}+\left[q_{l}\right]_{i}\left[q_{l}\right]_{j}-\delta_{i j} \hbar\right)$

The above expression is the classical limit of Eq. 4 because the mapping variables are no longer operators.

Now using Eq. 19 with the detailed expressions of $\Lambda_{l}$ (Eq. 20) and $\left[\hat{H}_{l}\right]_{\mathrm{W}}$ (Eq. 22), the full Liouvillian ${ }^{78}$ can be explicitly expressed as follows

$$
\begin{aligned}
\mathcal{L}^{[N]}= & \sum_{l=1}^{N}\left[\frac{P_{l}}{m} \frac{\vec{\partial}}{\partial R_{l}}-\left[V_{0}\left(R_{l}\right)+V_{\mathrm{e}}\left(R_{l}, \mathbf{q}_{l}, \mathbf{p}_{l}\right)\right] \frac{2}{\hbar} \sin \left(\frac{\hbar}{2} \frac{\overleftarrow{\partial}}{\partial R_{l}} \frac{\vec{\partial}}{\partial P_{l}}\right)\right. \\
& +\frac{1}{\hbar}\left[\mathbf{p}_{l}^{\mathrm{T}} \mathcal{V}\left(R_{l}\right) \vec{\nabla}_{\mathbf{q}_{l}}-\mathbf{q}_{l}^{\mathrm{T}} \mathcal{V}\left(R_{l}\right) \vec{\nabla}_{\mathbf{p}_{l}}\right] \cos \left(\frac{\hbar}{2} \frac{\overleftarrow{\partial}}{\partial R_{l}} \frac{\vec{\partial}}{\partial P_{l}}\right) \\
& \left.+\frac{1}{4}\left[\vec{\nabla}_{\mathbf{q}_{l}}^{\mathrm{T}} \mathcal{V}\left(R_{l}\right) \vec{\nabla}_{\mathbf{q}_{l}}+\vec{\nabla}_{\mathbf{p}_{l}}^{\mathrm{T}} \mathcal{V}\left(R_{l}\right) \vec{\nabla}_{\mathbf{p}_{l}}\right] \sin \left(\frac{\hbar}{2} \frac{\overleftarrow{\partial}}{\partial R_{l}} \frac{\vec{\partial}}{\partial P_{l}}\right)\right]
\end{aligned}
$$

The above Liouvillian was first derived in Ref. [ 78], and the detail of the derivation is provided in the Appendix $\mathrm{A}$ and the Supporting Information. Here, $V_{0}\left(R_{l}\right)$ and $V_{\mathrm{e}}\left(R_{l}, \mathbf{q}_{l}, \mathbf{p}_{l}\right)$ are defined in $\left[H_{l}\right]_{\mathrm{W}}\left(\right.$ Eq. 22), $\boldsymbol{\nabla}_{\mathbf{q}_{l}}$ and $\nabla_{\mathbf{p}_{l}}$ are the gradient operators corresponding to the $l_{\text {th }}$ mapping bead's position and momentum, respectively, as defined in Eq. 21. Further, $\mathcal{V}\left(\mathrm{R}_{l}\right)$ is the $(\mathcal{K} \times \mathcal{K})$ statedependent potential energy matrix, parametrically depends on $l_{\text {th }}$ nuclear bead's position as follow

$$
\mathcal{V}\left(\mathrm{R}_{l}\right)=\left(\begin{array}{cccc}
\mathcal{V}_{11}\left(R_{l}\right) & \mathcal{V}_{12}\left(R_{l}\right) & \ldots & \mathcal{V}_{1 \mathcal{K}}\left(R_{l}\right) \\
\vdots & \vdots & & \vdots \\
\mathcal{V}_{\mathcal{K} 1}\left(R_{l}\right) & \mathcal{V}_{\mathcal{K} 2}\left(R_{l}\right) & \ldots & \mathcal{V}_{\mathcal{K} \mathcal{K}}\left(\mathrm{R}_{l}\right)
\end{array}\right)
$$

This generalized Liouvillian $\mathcal{L}^{[N]}$ govern the time evolution of $N$ individual replicas connected through the zero-time quantum Boltzmann distribution. The exact Liouvillian $\mathcal{L}^{[N]}$ in Eq. 24 has three components. 
The first term, denotes as $\mathcal{L}_{\mathrm{n}}^{[N]}=\sum_{l=1}^{N} \frac{P_{l}}{m} \frac{\vec{\partial}}{\partial R_{l}}-$ $\left[V_{0}\left(R_{l}\right)+V_{\mathrm{e}}\left(R_{l}, \mathbf{q}_{l}, \mathbf{p}_{l}\right)\right] \frac{2}{\hbar} \sin \left(\frac{\hbar}{2} \frac{\overleftarrow{\partial}}{\partial R_{l}} \frac{\vec{\partial}}{\partial P_{l}}\right)$, corresponds to an Ehrenfest-type evolution of the nuclear DOF ${ }^{82}$ with higher-order nuclear derivatives (inside the sin function). The Second term in Eq. 24, denoted as $\mathcal{L}_{\mathrm{e}}^{[N]}=$ $\sum_{l=1}^{N} \frac{1}{\hbar}\left[\mathbf{p}_{l}^{\mathrm{T}} \mathcal{V}\left(R_{l}\right) \vec{\nabla}_{\mathbf{q}_{l}}-\mathbf{q}_{l}^{\mathrm{T}} \mathcal{V}\left(R_{l}\right) \vec{\nabla}_{\mathbf{p}_{l}}\right] \cos \left(\frac{\hbar}{2} \frac{\overleftarrow{\partial}}{\partial R_{l}} \frac{\vec{\partial}}{\partial P_{l}}\right)$, describes the electronic evolution with higher-order coupling terms to nuclear motions. The third term in Eq. 24, denoted as $\mathcal{L}_{\mathrm{h}}^{[N]}=\frac{1}{4}\left[\vec{\nabla}_{\mathbf{q}_{l}}^{\mathrm{T}} \mathcal{V}\left(R_{l}\right) \vec{\nabla}_{\mathbf{q}_{l}}+\right.$ $\left.\left.\vec{\nabla}_{\mathbf{p}_{l}}^{\mathrm{T}} \mathcal{V}\left(R_{l}\right) \vec{\nabla}_{\mathbf{p}_{l}}\right] \sin \left(\frac{\hbar}{2} \frac{\overleftarrow{\partial}}{\partial R_{l}} \frac{\vec{\partial}}{\partial P_{l}}\right)\right]$ contains coupled higherorder derivatives of nuclear and electronic motion. ${ }^{25,81,83}$ Note that there is no cross-bead interaction terms between electronic DOF and nuclear DOF in Eq. 24. Each individual bead term can only interacts with each other via $\mathcal{V}\left(R_{l}\right)$ (Eq. 25). If the system evolves only in a single surface (electronically adiabatic regime) or if $V_{e}\left(R_{l}, \mathbf{q}_{l}, \mathbf{p}_{l}\right)=0$, then Eq. 24 reduce down to conventional Wigner-Moyal series ${ }^{78-80}$ for $N$ independent replicas as follows

$$
\mathcal{L}^{[N]}=\sum_{l=1}^{N}\left[\frac{P_{l}}{m} \frac{\vec{\partial}}{\partial R_{l}}-\frac{2}{\hbar} V_{0}\left(R_{l}\right) \sin \left(\frac{\hbar}{2} \frac{\overleftarrow{\partial}}{\partial R_{l}} \frac{\vec{\partial}}{\partial P_{l}}\right)\right]
$$

On the other hand, if there is no nuclear DOF, then the Liouvillian (Eq. 24) becomes

$$
\mathcal{L}^{[N]}=\sum_{l=1}^{N} \frac{1}{\hbar}\left[\mathbf{p}_{l}^{\mathrm{T}} \mathcal{V} \vec{\nabla}_{\mathbf{q}_{l}}-\mathbf{q}_{l}^{\mathrm{T}} \mathcal{V} \vec{\nabla}_{\mathbf{p}_{l}}\right]
$$

It has been rigorously proved that the above Liouvillian preserves the electronic Rabi oscillation. ${ }^{78}$

\section{Time-Correlation Function}

With the detail expression of Liouvillian in Eq. 24 one can formally rewrite the Eq. 14 as

$$
\begin{aligned}
C_{A B}^{[N]}(t)= & \frac{\alpha_{N}}{\mathcal{Z}} \int \mathrm{d} \mathbf{R} \int \mathrm{d} \mathbf{P} \int \mathrm{d} \mathbf{q} \int \mathrm{d} \mathbf{p} \\
& \times\left[e^{-\beta \hat{H}} \hat{A}\right]_{\bar{N}} e^{\mathcal{L}^{[N]} t}[\hat{B}(0)]_{N},
\end{aligned}
$$

where $\mathcal{L}^{[N]}$ is the Liouvillian in Eq. $24,\left[e^{-\beta \hat{H}} \hat{A}\right]_{\bar{N}}$ is expressed in Eq. 15, and $[\hat{B}(0)]_{N}=\frac{1}{N} \sum_{k}\left[\hat{B}_{k}\right]_{\mathrm{W}}$ as shown in Eq. 17. Up to this point, there is no approximation in the expression of $C_{A B}^{[N]}(t)$ in Eq. 28.

We can further write $\left[e^{-\beta \hat{H}} \hat{A}\right]_{\bar{N}}$ (Eq. 15) into the sym- metric form as

$$
\begin{aligned}
& {\left[e^{-\beta \hat{H}} \hat{A}\right]_{\bar{N}}=\frac{1}{2}\left[\hat{A} e^{-\beta \hat{H}}+e^{-\beta \hat{H}} \hat{A}\right]_{\bar{N}} } \\
= & \frac{1}{N} \sum_{k=1}^{N} \int d \mathbf{D} \int d \boldsymbol{\Delta} \prod_{l=1}^{N} e^{\frac{i}{\hbar} P_{l} D_{l}} e^{\frac{i}{\hbar} \mathbf{p}_{l} \boldsymbol{\Delta}_{l}} \\
& \times \prod_{l \neq k}^{N}\left\langle\mathbf{q}_{l-1}-\frac{1}{2} \boldsymbol{\Delta}_{l-1}, R_{l-1}-\frac{1}{2} D_{l-1}\right| \hat{\mathcal{P}} e^{-\beta_{N} \hat{H}} \hat{\mathcal{P}} \\
& \times\left|\mathbf{q}_{l}+\frac{\boldsymbol{\Delta}_{l}}{2}, R_{l}+\frac{D_{l}}{2}\right\rangle\left\langle\mathbf{q}_{k-1}-\frac{1}{2} \boldsymbol{\Delta}_{k-1}, R_{k-1}-\frac{1}{2} D_{k-1}\right| \\
& \times \frac{1}{2}\left(\hat{\mathcal{P}} e^{-\beta_{N} \hat{H}} \hat{\mathcal{P}} \hat{A}+\hat{A} \hat{\mathcal{P}} e^{-\beta_{N} \hat{H}} \hat{\mathcal{P}}\right)\left|\mathbf{q}_{k}+\frac{\boldsymbol{\Delta}_{k}}{2}, R_{k}+\frac{D_{k}}{2}\right\rangle .
\end{aligned}
$$

Using the property of the Wigner transform, ${ }^{79,84}$ $\left[\hat{O}_{1} \hat{O}_{2}\right]_{\mathrm{W}}=\left[\hat{O}_{1}\right]_{\mathrm{W}} e^{-i \hat{\Lambda} \hbar / 2}\left[\hat{O}_{2}\right]_{\mathrm{W}}$, with $\hat{\Lambda}$ defined in Eq. 20, we can rewrite Eq. 29 as

$$
\left[e^{-\beta \hat{H}} \hat{A}\right]_{\bar{N}}=[\hat{A}]_{N}\left[\sum_{l=1}^{N} \cos \left(\hat{\Lambda}_{l} \hbar / 2\right)\right]\left[e^{-\beta \hat{H}}\right]_{\bar{N}},
$$

where $[\hat{A}]_{N}=\frac{1}{N} \sum_{k}\left[\hat{A}_{k}\right]_{\mathrm{W}}$, with

$$
\begin{aligned}
{\left[\hat{A}_{k}\right]_{\mathrm{W}} } & =\int d D_{k} \int d \boldsymbol{\Delta}_{k} e^{\frac{i}{\hbar} P_{k} D_{k}} e^{\frac{i}{\hbar} \mathbf{p}_{k} \boldsymbol{\Delta}_{k}} \\
& \times\left\langle\mathbf{q}_{k}-\frac{1}{2} \boldsymbol{\Delta}_{k}, R_{k}-\frac{1}{2} D_{k}|\hat{A}| \mathbf{q}_{k}+\frac{1}{2} \boldsymbol{\Delta}_{k}, R_{k}+\frac{1}{2} D_{k}\right\rangle,
\end{aligned}
$$

and $\hat{\Lambda}_{l}$ is defined previously in Eq. 20. The multidimentional Wigner transformed Boltzmann operator $\left[e^{-\beta \hat{H}}\right]_{\bar{N}}$ is expressed as

$$
\begin{aligned}
& {\left[e^{-\beta \hat{H}}\right]_{\bar{N}}=\int d \mathbf{D} \int d \boldsymbol{\Delta} \prod_{l=1}^{N} e^{\frac{i}{\hbar} P_{l} D_{l}} e^{\frac{i}{\hbar} \mathbf{p}_{l} \boldsymbol{\Delta}_{l}}} \\
& \quad \times\left\langle q_{l-1}-\frac{1}{2} \boldsymbol{\Delta}_{l-1}, R_{l-1}-\frac{1}{2} D_{l-1}\right| \hat{\mathcal{P}} e^{-\beta_{N} \hat{H}} \hat{\mathcal{P}} \\
& \quad \times\left|\mathbf{q}_{l}+\frac{1}{2} \boldsymbol{\Delta}_{l}, R_{l}+\frac{1}{2} D_{l}\right\rangle
\end{aligned}
$$

The detail derivation of Eq. 30 is provided in the supporting information.

To simplify our derivation, we further explore various special cases that can further simplify this equation. (i) If operator $\hat{A}$ is linear in nuclear position or momen$\operatorname{tum}(\hat{A}=\hat{R}$ or $\hat{A}=\hat{P})$, or (ii) if operator $\hat{A}$ and the Boltzmann operator $\left[e^{-\beta \hat{H}}\right]_{\bar{N}}$ act on different DOFs $(e g$, $\hat{A}$ contains electronic state $\left(\hat{A} \in \hat{\mathcal{P}}=\sum_{n}|n\rangle\langle n|\right)$ and in $\left[e^{-\beta \hat{H}_{0}}\right]_{\bar{N}}, \hat{H}_{0}$ only contains nuclear DOF), then for both (i) and (ii), only the first term in the cosine expansion will survive and hence we can write $\left[e^{-\beta \hat{H}} \hat{A}\right]_{\bar{N}}=$ $[\hat{A}]_{N}\left[\sum_{l=1}^{N} \cos \left(\hat{\Lambda}_{l} \hbar / 2\right)\right]\left[e^{-\beta \hat{H}}\right]_{\bar{N}}=[\hat{A}]_{N}\left[e^{-\beta \hat{H}}\right]_{\bar{N}}$. The detailed derivations for both case (i) and (ii) are provided in the Supporting Information.

From this point and below we assume that both operator $\hat{A}$ and $\hat{B}$ are linear function of the nuclear position $(R)$, such that we do not have to worry about the 
$\cos \left(\hat{\Lambda}_{l} \hbar / 2\right)$ operator in Eq.30. With this simplification, we can write the Eq. 28 as

$$
\begin{aligned}
C_{A B}^{[N]}(t)= & \frac{\alpha_{N}}{\mathcal{Z}} \int \mathrm{d} \mathbf{R} \int \mathrm{d} \mathbf{P} \int \mathrm{d} \mathbf{q} \int \mathrm{d} \mathbf{p} \\
& \times[\hat{A}]_{N}\left[e^{-\beta \hat{H}}\right]_{\bar{N}} e^{\mathcal{L}^{[N]} t}[\hat{B}]_{N} .
\end{aligned}
$$

\section{NORMAL MODE REPRESENTATION}

Here, we briefly introduced the normal mode coordinates of the free ring-polymer. The advantage of using normal modes is that with this set of global coordinates one can conveniently describe the collective motion of individual beads.

\section{A. Definition of the Normal Modes}

The free ring polymer Hamiltonian is defined as

$$
H^{\mathrm{RP}}=\sum_{l=1}^{N} \frac{P_{l}^{2}}{2 m}+\frac{m}{2 \beta_{N}^{2} \hbar^{2}}\left(R_{l}-R_{l-1}\right)^{2},
$$

where there is no external potential. Normal modes are defined as the eigenvectors of the Hessian matrix of $H_{\mathrm{rp}}$. Diagonalizing the Hessian matrix of $H_{\mathrm{rp}}$, one obtain the eigenvalues $\omega_{n}^{2}$, which are the square of following normal mode frequency

$$
\omega_{n}=\frac{2}{\beta_{N} \hbar} \sin \left(\frac{n \pi}{N}\right),
$$

where $n=0, \ldots, \pm(N-1) / 2$ is the index of normal modes. The same diagonalization process also gives the eigenvectors $T_{l n}$ of the Hessian matrix, which provides the relation between the primitive variables $R_{l}, P_{l}$ and the normal mode variable $\mathcal{Q}_{n}, \mathcal{P}_{n}$ as follows

$$
\begin{aligned}
\mathcal{Q}_{n} & =\sum_{l=1}^{N} \frac{T_{l n}}{\sqrt{N}} R_{l}, \mathcal{P}_{n}=\sum_{l=1}^{N} \frac{T_{l n}}{\sqrt{N}} P_{l} \\
R_{l} & =\sum_{n=-(N-1) / 2}^{(N-1) / 2} \sqrt{N} T_{l n} \mathcal{Q}_{n}, P_{l}=\sum_{n=-(N-1) / 2}^{(N-1) / 2} \sqrt{N} T_{l n} \mathcal{P}_{n}
\end{aligned}
$$

Similar transformation should also apply to $\left\{\mathcal{D}_{n}\right\}$ and $\left\{D_{l}\right\}$. The extra $\sqrt{N}$ factor in Eq. 36 ensures that the $\mathcal{Q}=\left\{\mathcal{Q}_{n}\right\}$ converges in the limit of $N \rightarrow \infty$. For an odd $N$ (to simplify our algebra), the transformation matrices are

$$
T_{l n}= \begin{cases}\sqrt{\frac{1}{N}} & n=0 \\ \sqrt{\frac{2}{N}} \sin (2 \pi l n / N) & n=1, \ldots,(N-1) / 2 \\ \sqrt{\frac{2}{N}} \cos (2 \pi l n / N) & n=-1, \ldots,-(N-1) / 2 .\end{cases}
$$

Under the normal mode representation, the free ring polymer Hamiltonian (Eq. 34) becomes

$$
H_{\mathrm{rp}}=\sum_{n=-(N-1) / 2}^{(N-1) / 2} \frac{\mathcal{P}_{n}{ }^{2}}{2 m}+\frac{1}{2} m \omega_{n}^{2} \mathcal{Q}_{n}^{2} .
$$

Note that the ring polymer spring terms are now the simplified uncoupled quadratic potentials, hence the normal modes of the free ring polymer can be evolved analytically by simple harmonic motion. ${ }^{85}$

\section{B. Time Correlation Function Under the Normal Mode Representation}

It is straightforward to transform Eq. 33 into the normal mode coordinates by using the orthonormal transformations defined in Eq. 36, leading to

$$
\begin{aligned}
& C_{A B}^{[N]}(t)=\frac{\alpha_{N}}{\mathcal{Z}} \int \mathrm{d} \mathbf{Q} \int \mathrm{d} \boldsymbol{P} \int \mathrm{d} \mathbf{q} \int \mathrm{d} \mathbf{p} \\
& \times[\hat{A}(0)]_{N}\left[e^{-\beta \hat{H}}\right]_{\bar{N}} e^{\mathcal{L}^{[N]} t}[\hat{B}(0)]_{N},
\end{aligned}
$$

where we used the shorthand notation $\int d \mathcal{Q}=$ $\prod_{n=-(N-1) / 2}^{(N-1) / 2} \int d \mathcal{Q}_{n}$ and likewise for $\int d \mathcal{P}$. Here, both the $\int d \mathbf{q}$ and $\int d \mathbf{p}$ are defined as before in beads representation, such as $\int d \mathbf{q}=\prod_{l=1}^{N} \int d \mathbf{q}_{l}$ and likewise for $\int d \mathbf{p}$. Only nuclear coordinates $\mathbf{R}$ and $\mathbf{P}$ are transformed to their corresponding normal mode coordinates $\mathcal{Q}$ and $\mathcal{P}$, respectively.

Using new coordinates $\xi_{l}^{ \pm}$defined as follows

$$
\xi_{l}^{ \pm}=\sum_{n=-(N-1) / 2}^{(N-1) / 2} \sqrt{N} T_{l n} \mathcal{Q}_{n} \pm \sum_{n=-(N-1) / 2}^{(N-1) / 2} \frac{1}{2} \sqrt{N} T_{l n} \mathcal{D}_{n}
$$

$\left[e^{-\beta \hat{H}}\right]_{\bar{N}}$ is expressed in these normal mode coordinates as

$$
\begin{aligned}
& {\left[e^{-\beta \hat{H}}\right]_{\bar{N}}=\int d \mathcal{D} \int d \boldsymbol{\Delta}\left[\prod_{n=-(N-1) / 2}^{(N-1) / 2} e^{\frac{i}{\hbar} N \mathcal{P}_{n} \mathcal{D}_{n}}\right] \prod_{l=1}^{N} e^{\frac{i}{\hbar} p_{l} \Delta_{l}}} \\
& \times\left\langle\mathbf{q}_{l-1}-\frac{1}{2} \boldsymbol{\Delta}_{l-1}, \xi_{l-1}^{-}\left|\hat{\mathcal{P}} e^{-\beta_{N} \hat{H}} \hat{\mathcal{P}}\right| \mathbf{q}_{l}+\frac{\boldsymbol{\Delta}_{l}}{2}, \xi_{l}^{+}\right\rangle
\end{aligned}
$$

Providing the operators $\hat{A}=\hat{B}=\hat{R},[\hat{A}]_{N}$ can be expressed in normal mode coordinates as

$$
[\hat{A}]_{N}=\frac{1}{N} \sum_{k=1}^{N}\left[\hat{A}_{k}\right]_{\mathrm{W}}=\frac{1}{N} \sum_{k=1}^{N} R_{k}=\frac{1}{N} \sum_{k=1}^{N} \sum_{n} \sqrt{N} T_{k n} \mathcal{Q}_{n},
$$

and same for $[\hat{B}]_{N}$. In the last line of Eq. 42, we have substitute $R_{k}$ with the corresponding normal mode transformation defined in Eq. 36 .

Finally, we decompose the total Liouvillian $\mathcal{L}^{[N]}$ (Eq. 24) into the following terms

$$
\mathcal{L}^{[N]}=\mathcal{L}_{\mathrm{n}}^{[N]}+\mathcal{L}_{\mathrm{e}}^{[N]}+\mathcal{L}_{\mathrm{h}}^{[N]},
$$


and express each term with the normal mode coordinates

$$
\begin{aligned}
\mathcal{L}_{\mathrm{n}}^{[N]}= & \sum_{n=-(N-1) / 2}^{(N-1) / 2}\left(\frac{\mathcal{P}_{n}}{m} \frac{\vec{\partial}}{\partial \mathcal{Q}_{n}}\right) \\
- & \frac{2 N}{\hbar}\left[U_{0}^{[N]}(\mathcal{Q})+U_{e}^{[N]}(\mathcal{Q}, \mathbf{q}, \mathbf{p})\right] \\
& \times \sin \left(\frac{\hbar}{2 N} \sum_{n=-(N-1) / 2}^{(N-1) / 2} \frac{\overleftarrow{\partial}}{\partial \mathcal{Q}_{n}} \frac{\vec{\partial}}{\partial \mathcal{P}_{n}}\right) \\
\mathcal{L}_{\mathrm{e}}^{[N]}= & \frac{1}{\hbar} \sum_{l=1}^{N}\left\{\left[\mathbf{p}_{l}^{\mathrm{T}} \mathcal{V}\left(R_{l}(\mathcal{Q})\right) \vec{\nabla}_{\mathbf{q}_{l}}-\mathbf{q}_{l}^{\mathrm{T}} \mathcal{V}\left(R_{l}(\mathcal{Q})\right) \vec{\nabla}_{\mathbf{p}_{l}}\right]\right\} \\
& \times \cos \left(\frac{\hbar}{2 N} \sum_{n=-(N-1) / 2}^{(N-1) / 2} \frac{\overleftarrow{\partial}}{\partial \mathcal{Q}_{n}} \frac{\vec{\partial}}{\partial \mathcal{P}_{n}}\right) \\
\mathcal{L}_{\mathrm{h}}^{[N]}= & \frac{1}{4} \sum_{l=1}^{N}\left\{\left[\vec{\nabla}_{\mathbf{q}_{l}}^{\mathrm{T}} \mathcal{V}\left(R_{l}(\mathcal{Q})\right) \vec{\nabla}_{\mathbf{q}_{l}}+\vec{\nabla}_{\mathbf{p}_{l}}^{\mathrm{T}} \mathcal{V}\left(R_{l}(\mathcal{Q})\right) \vec{\nabla}_{\left.\mathbf{p}_{l}\right]}\right]\right\} \\
& \times \sin \left(\frac{\hbar}{2 N} \sum_{n=-(N-1) / 2}^{(N-1) / 2} \frac{\overleftarrow{\partial}}{\partial \mathcal{Q}_{n}} \frac{\vec{\partial}_{\partial}}{\partial \mathcal{P}_{n}}\right)
\end{aligned}
$$

Here, normal mode representation of $U_{0}^{[N]}(\mathcal{Q})$, $U_{e}^{[N]}(\mathcal{Q}, \mathbf{q}, \mathbf{p})$ and $\mathcal{V}\left(R_{l}(\mathcal{Q})\right)$ are defined as

$$
\begin{array}{r}
U_{0}^{[N]}(\mathcal{Q})=\frac{1}{N} \sum_{l=1}^{N} V_{0}\left(\sum_{n=-(N-1) / 2}^{(N-1) / 2} \sqrt{N} T_{l n} \mathcal{Q}_{n}\right) \\
U_{e}^{[N]}(\mathcal{Q}, \mathbf{q}, \mathbf{p})=\frac{1}{N} \sum_{l=1}^{N} \sum_{i, j=1}^{\mathcal{K}} \mathcal{V}_{i j}\left(\sum_{n=-(N-1) / 2}^{(N-1) / 2} \sqrt{N} T_{l n} \mathcal{Q}_{n}\right) \\
\times \frac{1}{2 \hbar}\left(\left[p_{i}\right]_{l}\left[p_{j}\right]_{l}+\left[q_{i}\right]_{l}\left[q_{j}\right]_{l}-\delta_{i j} \hbar\right) \\
\mathcal{V}\left(R_{l}(\mathcal{Q})\right)=\left(\begin{array}{cccc}
\mathcal{V}_{11}\left(R_{l}(\mathcal{Q})\right) & \mathcal{V}_{12}\left(R_{l}(\mathcal{Q})\right) & \ldots & \mathcal{V}_{1 \mathcal{K}}\left(R_{l}(\mathcal{Q})\right) \\
\vdots & \vdots & \\
\mathcal{V}_{\mathcal{K} 1}\left(R_{l}(\mathcal{Q})\right) & \mathcal{V}_{\mathcal{K} 2}\left(R_{l}(\mathcal{Q})\right) & \ldots & \mathcal{V}_{\mathcal{K} \mathcal{K}}\left(R_{l}(\mathcal{Q})\right)
\end{array}\right)
\end{array}
$$

where each $\mathcal{V}_{i j}\left(R_{l}(\mathcal{Q})\right)$ term is

$$
\mathcal{V}_{i j}\left(R_{l}(\mathcal{Q})\right)=\mathcal{V}_{i j}\left(\sum_{n=-(N-1) / 2}^{(N-1) / 2} \sqrt{N} T_{l n} \mathcal{Q}_{n}\right),
$$

Note that in Eq. 50, the $\mathcal{V}_{i j}\left(R_{l}(\boldsymbol{Q})\right)$ term corresponds to the diabatic potential $\mathcal{V}_{i j}(R)$ evaluated at the $l_{\text {th }}$ bead position $R_{l}$ in terms of the normal mode coordinate $R_{l}=\sum_{n} \sqrt{N} T_{l n} \mathcal{Q}_{n}$. Further, each nuclear derivative term $\frac{\overleftarrow{\partial}}{\partial \mathcal{Q}_{n}} \frac{\vec{\partial}}{\partial \mathcal{P}_{n}}$ in the above Liouvillian does not couple to each other. They only couple to each other through the sine or cosine function in the Liouvillian.

\section{NON-ADIABATIC MATSUBARA DYNAMICS}

\section{A. Matsubara Modes}

We now considering $M$ lowest frequency normal modes for $M \ll N$, under the limit $N \rightarrow \infty$. They are commonly referred to as the Matsubara modes ${ }^{38,69}$ of distinguishable particles, with the corresponding Matsubara frequency $\tilde{\omega}_{n}$ as follows

$$
\tilde{\omega}_{n}=\lim _{N \rightarrow \infty} \omega_{n}=\frac{2 n \pi}{\beta \hbar} .
$$

The superposition of these $M$ Matsubara modes produces a smooth and differentiable function ${ }^{69}$ in imaginary time $\tau$, such that $R_{l}=R(\tau)$ for $\tau=\beta_{N} \hbar l$ with $l=1, \ldots, N$. This means that one can construct the smooth imaginary-time path $\mathrm{R}(\tau)$ from the Matsubara modes $^{69,86-88}$ as follows

$R(\tau)=\mathcal{Q}_{0}+\sqrt{2} \sum_{n=-(M-1) / 2}^{(M-1) / 2}\left[\sin \left(\tilde{\omega}_{n} \tau\right) \mathcal{Q}_{n}+\cos \left(\tilde{\omega}_{n} \tau\right) \mathcal{Q}_{-n}\right]$,

and the same relations for $P(\tau)$ and $D(\tau)$. The significance of Matsubara modes is that their superposition generate smooth and differentiable functions of the imaginary-time $\tau$. On the other hand, if one consider both the Matsubara $(M)$ and non-Matsubara $(N$ $M)$ modes, then the imaginary-time path is not necessarily differentiable, because the latter give rise to nonsmooth, non-differentiable distribution with respect to $\tau$. It is a well-known fact that the Boltzmann operator guarantees that only the Matsubara modes contribute to the initial Quantum Boltzmann Distribution (QBD) ${ }^{38,89-91}$ (for electronically adiabatic systems). Moreover, the previous work on Matsubara dynamics ${ }^{69,73}$ have suggest that there is a close connection between the smoothness in imaginary-time and the dynamics that preserves the QBD.

Similar to the previous work of the Matsubara dynamics ${ }^{69}$ here, we show that (in Section IV. D) one can integrate out all of the non-Matsubara modes from Eq. 39, giving rise to the exact initial quantum statistics (which corresponds to the generalized Kubo transformed correlation function at $t=0)$ in the limit $M \rightarrow \infty$, $M \ll N^{69,89-91}$. This suggest that only the smooth and imaginary-time differentiable Matsubara modes contributes to the initial quantum statistics for the electronically non-adiabatic systems as well. This makes one wondering how important are those non-Matsubara modes in quantum dynamics (Liouvillian), since they do not contribute to the initial QBD at all. ${ }^{69}$ We will explicitly discuss such an approximation that drops the nonMatsubara modes in Liouvillian ${ }^{69}$ in the next section. 


\section{B. Matsubara Approximation}

We separate each term in Eq. 43 into two parts, one contains the lowest $M$ Matsubara modes, and the other contains $(N-M)$ non-Matsubara modes

$$
\mathcal{L}^{[N]}=\mathcal{L}^{[M]}+\mathcal{L}^{[N-M]}
$$

where the Matsubara Liouvillian is $\mathcal{L}^{[M]}$, and the nonMatsubara Liouvillian $\mathcal{L}^{[N-M]}$ is expressed in Appendix A. Note that $\mathcal{L}^{[N-M]}$ does not contain any derivatives with respect to the mapping variables, hence there is no direct influence from $\mathcal{L}^{[N-M]}$ to the electronic subsystem. The non-Matsubara Liouvillian $\mathcal{L}^{[N-M]}$, on the other hand, does couple the non-Matsubara modes with the Matsubara mode, where the Matsubara mode couple to the mapping DOFs through the Matsubara Liouvillian $\mathcal{L}^{[M]}$.

Since the non-Matsubara modes do not contribute to the initial quantum statistics, it might also be a good approximation (at least in short time) to ignore their presence in the quantum Liouvillian. ${ }^{69}$ The Matsubara dynamics assumes that the time evolution of nuclei are only governed by the smooth Matsubara modes instead of all normal modes. This approximation is achieved by neglecting the non-Matsubara modes in the derivatives of the corresponding Liouvilian terms $\mathcal{L}^{[N]} \approx$ $\mathcal{L}^{[M]}+\mathcal{O}\left(\mathcal{L}^{[N-M]}\right)$, which effectively produces the decoupling between non-Matsubara modes from the Matsubara modes during the time evaluation. Under the limit $N \rightarrow \infty$, one discards the $(N-M)$ higher frequency, non-smooth modes. ${ }^{69,71,73}$

Similar to $\mathcal{L}^{[N]}$ in Eq. 43, we further decompose $\mathcal{L}^{[M]}$ into the following three terms

$$
\mathcal{L}^{[M]}=\mathcal{L}_{\mathrm{n}}^{[M]}+\mathcal{L}_{\mathrm{e}}^{[M]}+\mathcal{L}_{\mathrm{h}}^{[M]},
$$

where the detailed expressions for each term are

$$
\begin{aligned}
& \mathcal{L}_{\mathrm{n}}^{[M]}=\sum_{n=-(M-1) / 2}^{(M-1) / 2}\left(\frac{\mathcal{P}_{n}}{m} \frac{\vec{\partial}}{\partial \mathcal{Q}_{n}}\right) \\
& -\frac{2 N}{\hbar}\left[U_{0}^{[N]}(\mathcal{Q})+U_{e}^{[N]}(\boldsymbol{Q}, \mathbf{q}, \mathbf{p})\right] \\
& \times \sin \left(\frac{\hbar}{2 N} \sum_{n=-(M-1) / 2}^{(M-1) / 2} \frac{\overleftarrow{\partial}}{\partial \mathcal{Q}_{n}} \frac{\vec{\partial}}{\partial \mathcal{P}_{n}}\right) \\
& \mathcal{L}_{\mathrm{e}}^{[M]}=\frac{1}{\hbar} \sum_{l=1}^{N}\left\{\left[\mathbf{p}_{l}^{\mathrm{T}} \mathcal{V}\left(R_{l}(\mathcal{Q})\right) \vec{\nabla}_{\mathbf{q}_{l}}-\mathbf{q}_{l}^{\mathrm{T}} \mathcal{V}\left(R_{l}(\mathcal{Q})\right) \vec{\nabla}_{\mathbf{p}_{l}}\right]\right\} \\
& \times \cos \left(\frac{\hbar}{2 N} \sum_{n=-(M-1) / 2}^{(M-1) / 2} \frac{\overleftarrow{\partial}}{\partial \mathcal{Q}_{n}} \frac{\vec{\partial}}{\partial \mathcal{P}_{n}}\right) \\
& \mathcal{L}_{\mathrm{h}}^{[M]}=\frac{1}{4} \sum_{l=1}^{N}\left\{\left[\vec{\nabla}_{\mathbf{q}_{l}}^{\mathrm{T}} \mathcal{V}\left(R_{l}(\mathcal{Q})\right) \vec{\nabla}_{\mathbf{q}_{l}}+\vec{\nabla}_{\mathbf{p}_{l}}^{\mathrm{T}} \mathcal{V}\left(R_{l}(\mathcal{Q})\right) \vec{\nabla}_{\mathrm{p}_{l}}\right]\right\} \\
& \times \sin \left(\frac{\hbar}{2 N} \sum_{n=-(M-1) / 2}^{(M-1) / 2} \frac{\overleftarrow{\partial}}{\partial \mathcal{Q}_{n}} \frac{\vec{\partial}}{\partial \mathcal{P}_{n}}\right)
\end{aligned}
$$

In Eq. 55 to Eq. 57, we re-express $\mathcal{L}_{\mathrm{n}}^{[N]}, \mathcal{L}_{\mathrm{e}}^{[N]}$ and $\mathcal{L}_{\mathrm{h}}^{[N]}$ in Matsubara modes under the $N \rightarrow \infty$ and $M \ll N$ limit. It is worth mentioning that the full Matsubara Liouvillian in Eq. 54 contains potential terms $U_{0}^{[N]}(\mathcal{Q})$, $U_{e}^{[N]}(\mathcal{Q}, \mathbf{q}, \mathbf{p})$, and $\mathcal{V}\left(R_{l}(\mathcal{Q})\right)$, which still depend on all $N$ normal modes. On the other hand, all derivatives only involve $M$ Matsubara modes.

\section{Matsubara Time-Correlation Function}

Explicitly applying the Matsubara approximation for the Liouvillian $\mathcal{L}^{[N]} \approx \mathcal{L}^{[M]}+\mathcal{O}\left(\mathcal{L}^{[N-M]}\right)$, the exact correlation function in Eq. 39 becomes an approximate correlation function $C_{A B}^{[N]}(t) \approx C_{A B}^{[M]}(t)$, whereas $C_{A B}^{[N]}(0)=$ $C_{A B}^{[M]}(0)$ such that the initial QBD is exactly captured (see Section D). This approximate TCF, which is commonly referred to as the Matsubara TCF, is expressed as follows

$$
\begin{aligned}
& C_{A B}^{[M]}(t)=\lim _{N \rightarrow \infty} \frac{\alpha_{N}}{\mathcal{Z}} \int d \mathcal{Q} \int d \mathcal{P} \int d \mathbf{q} \int d \mathbf{p} \\
& \times[\hat{A}(0)]_{N}\left[e^{-\beta \hat{H}}\right]_{\bar{N}} e^{\mathcal{L}^{[M]} t}[\hat{B}(0)]_{N} .
\end{aligned}
$$

Note that the above expression still depends on the non-Matsubara modes through the potentials in $\mathcal{L}^{[M]}$ and the QBD term $\left[e^{-\beta \hat{H}}\right]_{\bar{N}}$, and the integral $\int d \mathcal{Q}$ and $\int d \mathcal{P}$ still include all normal modes (Matsubara and nonMatsubara). However, as non-Matsubara modes are decoupled from the Matsubara modes (because we have dropped $\mathcal{L}^{[N-M]}$ in the Liouvillian), one can analytically integrated out all of the non-Matsubara modes under the limit of $N \rightarrow \infty, M \rightarrow \infty$, and $M \ll N$. The detailed derivation of this procedure is provided in Appendix C.

After integrating out the non-Matsubara modes in Eq. 58, we reach to the first key result of this paper as follows

$$
\begin{aligned}
& C_{A B}^{[M]}(t)=\frac{\alpha_{N} \cdot \alpha_{M}}{\mathcal{Z}_{M}} \int d \boldsymbol{\mathcal { Q }}_{M} \int d \boldsymbol{P}_{M} \int d \mathbf{q} \int d \mathbf{p} A\left(\mathcal{Q}_{M}\right) \\
& \times \Gamma\left(\mathcal{Q}_{M}, \mathbf{q}, \mathbf{p}\right) e^{-\beta\left(H_{M}\left(\mathcal{P}_{M}, \mathcal{Q}_{M}\right)-i \theta_{M}\left(\mathcal{P}_{M}, \mathcal{Q}_{M}\right)\right)} e^{\mathcal{L}^{[M]} t} B\left(\boldsymbol{\mathcal { Q }}_{M}\right),
\end{aligned}
$$

where the shorthand notations for the integrals are $\int d \mathcal{Q}_{M}=\prod_{n=-(M-1) / 2}^{(M-1) / 2} d \mathcal{Q}_{n}, \int d \mathbf{q}=\prod_{l=1}^{N} d q_{l}$, and analogously for $\int d \boldsymbol{P}_{M}$ and $\int d \mathbf{p}$, and $\alpha_{M}$ is the following constant

$$
\alpha_{M}=\frac{\hbar^{(1-M)}}{[(M-1) / 2] !^{2}} .
$$

Note that the above correlation function $C_{A B}^{[M]}(t)$ is explicitly depends on the Matsubara modes $\mathcal{Q}_{M}$ and $\mathcal{P}_{M}$. The mapping DOFs, on the other hand, are still expressed in the primitive (bead) coordinates with all $N$ copies, as we did not make any approximation on them. The Liouvillian $\mathcal{L}^{[M]}$ has the same 
expression in Eq. 55-Eq. 57 but with following substitutions $U_{0}^{[N]}(\boldsymbol{\mathcal { Q }}) \rightarrow U_{0}^{[M]}\left(\boldsymbol{\mathcal { Q }}_{M}\right), \quad U_{e}^{[N]}(\mathcal{Q}, \mathbf{q}, \mathbf{p}) \rightarrow$ $U_{e}^{[M]}\left(\mathcal{Q}_{M}, \mathbf{q}, \mathbf{p}\right)$, and $\mathcal{V}\left(R_{l}(\mathcal{Q})\right) \rightarrow \mathcal{V}\left(R_{l}\left(\mathcal{Q}_{M}\right)\right)$, whereas these new potential only contains the Matsubara modes, for example

$$
U_{0}^{[M]}\left(\mathcal{Q}_{M}\right)=\lim _{N \rightarrow \infty} \frac{1}{N} \sum_{l=1}^{N} V_{0}\left(\sum_{n=-(M-1) / 2}^{(M-1) / 2} T_{l n} \sqrt{N} \mathcal{Q}_{n}\right) .
$$

and similarly for $U_{e}^{[M]}\left(\mathcal{Q}_{M}, \mathbf{q}, \mathbf{p}\right)$ and $\mathcal{V}\left(R_{l}\left(\mathcal{Q}_{M}\right)\right)$ by replacing the sum in Eq. 48 and Eq. 49 from originally over all modes to the sum over only the Matsubara modes. Note that the nuclear coordinate $R_{l}\left(\mathcal{Q}_{M}\right)=$ $\sum_{n=-(M-1) / 2}^{(M-1) / 2} T_{l n} \sqrt{N} \mathcal{Q}_{n}$ is different than the original coordinate $R_{l}(\mathcal{Q})=\sum_{n=-(N-1) / 2}^{(N-1) / 2} T_{l n} \sqrt{N} \mathcal{Q}_{n}$; the former one only contains smooth (and imaginary-time differentiable) Matsubara mode, and later one contains all modes.

Further, $H_{M}\left(\mathcal{P}_{M}, \mathcal{Q}_{M}\right)$ is expressed as

$$
H_{M}\left(\mathcal{P}_{M}, \mathcal{Q}_{M}\right)=\sum_{n=-(M-1) / 2}^{(M-1) / 2} \frac{\mathcal{P}_{n}^{2}}{2 m}+U_{0}^{[M]}\left(\boldsymbol{Q}_{M}\right),
$$

and the Matsubara phase $\theta_{M}$ takes the following form

$$
\theta_{M}\left(\mathcal{P}_{M}, \mathcal{Q}_{M}\right)=\sum_{n=-(M-1) / 2}^{(M-1) / 2} \mathcal{P}_{n} \tilde{\omega}_{n} \mathcal{Q}_{-n}
$$

where $\tilde{\omega}_{n}=2 n \pi / \beta \hbar$ is the Matsubara frequency (Eq. 51).

The $\Gamma\left(\mathcal{Q}_{M}, \mathbf{q}, \mathbf{p}\right)$ term in Eq. 59 corresponds to the QBD originated from the electronic-nuclear interaction, which is expressed as follows

$\Gamma\left(\mathcal{Q}_{M}, \mathbf{q}, \mathbf{p}\right)=\phi \cdot e^{-\frac{\mathcal{G}_{N}}{\hbar}} \operatorname{Tr}_{\mathrm{e}}\left[\prod_{l=1}^{N}\left(\mathbf{C}_{l}-\frac{1}{2} \mathcal{I}\right) \mathcal{M}\left(R_{l}\left(\mathcal{Q}_{M}\right)\right)\right]$

where $\phi=2^{(\mathcal{K}+1) N} / \hbar^{N}$, with $\mathcal{G}_{N}=\sum_{l=1}^{N}\left(\mathbf{q}_{l} \mathbf{q}_{l}^{\mathrm{T}}+\mathbf{p}_{l} \mathbf{p}_{l}^{\mathrm{T}}\right)$. Further, $\mathbf{C}_{l}$ is expressed as

$$
\mathbf{C}_{l}=\left(\mathbf{q}_{l}+i \mathbf{p}_{l}\right) \times\left(\mathbf{q}_{l}-i \mathbf{p}_{l}\right)^{\mathrm{T}},
$$

and $\mathcal{I}$ is the $(\mathcal{K} \times \mathcal{K})$ identity matrix. Note that $\left(\mathbf{C}_{l}-\frac{1}{2} \mathcal{I}\right)$ can be interpreted as the reduced density matrix associated with the $l_{\text {th }}$ bead. In addition, $\mathcal{M}_{i j}\left(R_{l}\left(\boldsymbol{\mathcal { Q }}_{M}\right)\right)$ is the matrix element of the electronic Boltzmann operator expressed as follows

$$
\mathcal{M}_{i j}\left(R_{l}\left(\boldsymbol{\mathcal { Q }}_{M}\right)\right)=\left\langle i\left|e^{-\beta_{N} \hat{V}_{e}\left(R_{l}\left(\mathcal{Q}_{M}\right)\right)}\right| j\right\rangle,
$$

where $\hat{V}_{e}\left(R_{l}\left(\mathcal{Q}_{M}\right)\right)=\sum_{i j} \mathcal{V}_{i j}\left(R_{l}\left(\mathcal{Q}_{M}\right)\right)|i\rangle\langle j|$ is the statedependent potential operator evaluated at $l_{\text {th }}$ bead position $R_{l}$ in terms of the Matsubara coordinate, $R_{l}=$ $\sum_{n=-(M-1) / 2}^{(M-1) / 2} \sqrt{N} T_{l n} \mathcal{Q}_{n}$. The expression of $\Gamma$ was derived in the MV-RPMD partition function expression. ${ }^{57}$
Finally, the partition function is expressed as

$$
\begin{aligned}
\mathcal{Z}_{M}= & \alpha_{N} \cdot \alpha_{M} \int d \boldsymbol{Q}_{M} \int d \boldsymbol{\mathcal { P }}_{M} \int d \mathbf{q} \int d \mathbf{p} \\
& \times \Gamma\left(\boldsymbol{Q}_{M}, \mathbf{q}, \mathbf{p}\right) e^{-\beta\left(H_{M}\left(\boldsymbol{P}_{M}, \mathcal{Q}_{M}\right)-i \theta_{M}\left(\boldsymbol{P}_{M}, \boldsymbol{Q}_{M}\right)\right)}
\end{aligned}
$$

Note that under the Matsubara limit $N \rightarrow \infty, M \rightarrow$ $\infty$, and $M \ll N$, one can further Taylor expand the sine and cosine terms in Eq. 55 to Eq. 57 as follow

$$
\begin{aligned}
\lim _{N \rightarrow \infty} \lim _{M \ll N} \frac{2 N}{\hbar} \sin \left(\frac{\hbar}{2 N} \sum_{n=-(M-1) / 2}^{(M-1) / 2} \frac{\overleftarrow{\partial}}{\partial \mathcal{Q}_{n}} \frac{\vec{\partial}}{\partial \mathcal{P}_{n}}\right) \\
=\sum_{n=-(M-1) / 2} \frac{\overleftarrow{\partial}}{\partial \mathcal{Q}_{n}} \frac{\vec{\partial}}{\partial \mathcal{P}_{n}}+\mathcal{O}\left(\frac{M^{3} \hbar^{2}}{N^{2}}\right) \\
\lim _{N \rightarrow \infty} \lim _{M \ll N} \cos \left(\frac{\hbar}{2 N} \sum_{n=-(M-1) / 2}^{(M-1) / 2} \frac{\overleftarrow{\partial}}{\partial \mathcal{Q}_{n}} \frac{\vec{\partial}}{\partial \mathcal{P}_{n}}\right) \\
=1+\mathcal{O}\left(\frac{M^{2} \hbar^{2}}{N^{2}}\right)
\end{aligned}
$$

From the above analysis, it is clear that in the Matsubara space, the "effective" Planck constant inside the cos term is re-scaled as

$$
\hbar \rightarrow \frac{M}{N} \hbar,
$$

and as $\sqrt{M} \frac{M}{N} \hbar$ for the sin term. ${ }^{70}$ Thus, it can be made as small as desired by increasing $N$. Hence, truncating Eq. 55 to 57 to the first order of $\frac{\overleftarrow{\partial}}{\partial \mathcal{Q}_{n}} \frac{\vec{\partial}}{\partial \mathcal{P}_{n}}$ in the Matsubara space becomes exact. Further, the $\mathcal{L}_{\mathrm{h}}^{[M]}$ term (Eq. 57) is on the order of $\sim \mathcal{O}\left(\frac{M}{N} \hbar\right)$, thus can be completely ignored under the Matsubara limit.

These effective scaling of the Planck constant is the main advantage of the Matsubara dynamics, compared to the previous approaches (see Appendix D) that rely on the truncation of the Liouvillian based on the argument of small $\hbar$, which may or may not be a good approximation. Also, note that the argument in Eq. 55 and 57 does not work for non-Matsubara modes, as the error term becomes $\mathcal{O}\left((N-M)^{3} \hbar^{2} / N^{2}\right)$ for Eq. 67 and $\mathcal{O}\left((N-M)^{2} \hbar^{2} / N^{2}\right)$ for Eq. 68, which are no longer small under the $N \rightarrow \infty$ limit.

Therefore, under the Matsubara limit, we can exactly expressed the original Matsubara Liouvillian $\mathcal{L}^{[M]}$ (with the expression of Eq. 55 -Eq. 57 with $\mathcal{Q}_{M}$ ) into the following equivalent expression

$$
\begin{aligned}
\mathcal{L}^{[M]}= & \sum_{n=-(M-1) / 2}^{(M-1) / 2} \frac{\mathcal{P}_{n}}{m} \frac{\vec{\partial}}{\partial \mathcal{Q}_{n}} \\
- & \sum_{n=-(M-1) / 2}^{(M-1) / 2}\left(\frac{\partial U_{0}^{[M]}\left(\boldsymbol{Q}_{M}\right)}{\partial \mathrm{Q}_{n}}+\frac{\partial U_{e}^{[M]}\left(\mathcal{Q}_{M}, \mathbf{q}, \mathbf{p}\right)}{\partial \mathrm{Q}_{n}}\right) \frac{\vec{\partial}}{\partial \mathcal{P}_{n}} \\
& +\frac{1}{\hbar} \sum_{l=1}^{N}\left(\mathbf{p}_{l}^{\mathrm{T}} \mathcal{V}\left(R_{l}\left(\boldsymbol{Q}_{M}\right)\right) \vec{\nabla}_{\mathbf{q}_{l}}-\mathbf{q}_{l}^{\mathrm{T}} \mathcal{V}\left(R_{l}\left(\mathcal{Q}_{M}\right)\right) \vec{\nabla}_{\mathbf{p}_{l}}\right),
\end{aligned}
$$


where $U_{e}^{[M]}\left(\mathcal{Q}_{M}, \mathbf{q}, \mathbf{p}\right)$ and $\mathcal{V}\left(R_{l}\left(\mathcal{Q}_{M}\right)\right)$ are defined analogously as those in Eq. 48 and Eq. 49, respectively, where $\mathcal{Q}_{M}$ are normal modes in the "Matsubara" domain, $[-(M-1) / 2 \ldots(M-1) / 2]$, such that $R_{l}\left(\mathcal{Q}_{M}\right)=$ $\sum_{n=-(M-1) / 2}^{(M-1) / 2} T_{l n} \sqrt{N} \mathcal{Q}_{n}$.

In the above Matsubara Liouvillian, we have explicitly dropped the following higher order term (in $\mathcal{L}^{[M]}$, Eq. 54)

$$
\begin{array}{r}
\mathcal{L}_{\mathrm{h}}^{[M]}=\frac{1}{8} \frac{\hbar}{N} \sum_{n=-(M-1) / 2}^{(M-1) / 2} \sum_{l=1}^{N}\left(\left[\vec{\nabla}_{\mathbf{q}_{l}^{\mathrm{T}}} \frac{\partial \mathcal{V}\left(R_{l}\left(\boldsymbol{\mathcal { Q }}_{M}\right)\right)}{\partial \mathcal{Q}_{n}} \vec{\nabla}_{\mathbf{q}_{l}}\right.\right. \\
\left.\left.+\vec{\nabla}_{\mathbf{p}_{l}}^{\mathrm{T}} \frac{\partial \mathcal{V}\left(R_{l}\left(\boldsymbol{\mathcal { Q }}_{M}\right)\right)}{\partial \mathcal{Q}_{n}} \vec{\nabla}_{\mathbf{p}_{l}}\right]\right) \frac{\vec{\partial}}{\partial \mathcal{P}_{n}}
\end{array}
$$

where $\frac{\partial \mathcal{V}\left(R_{l}\left(\mathcal{Q}_{M}\right)\right)}{\partial \mathcal{Q}_{n}}$ is a $(\mathcal{K} \times \mathcal{K})$ matrix, with the matrix element $\frac{\partial \mathcal{V}_{i j}\left(R_{l}\left(\mathcal{Q}_{M}\right)\right)}{\partial \mathcal{Q}_{n}}$. This term is in the order of $\mathcal{O}\left(\frac{M}{N} \frac{1}{\hbar}\right)$ and hence can also be made as small as desired under the Matsubara limit. Note that this term accounts for the back action from the electronic subsystem to the nuclear DOF. ${ }^{81}$ It has been shown that this term can be transformed into a local potential on nuclear DOF, leading to a non-Hamiltonian Liouvillian in Possion Bracket Mapping Equation (PBME), which can further improve the population dynamics. ${ }^{83}$ In the non-adiabatic Matsubara dynamics, this term can be dropped which will not introduce and additional error beyond the order of $\mathcal{O}\left(\frac{M}{N} \frac{1}{\hbar}\right)$.

The Matsubara correlation function in Eq. 59 contains an imaginary phase factor $\theta_{M}$, which potentially introduces a sign problem for a system that contains multidimensional nuclear DOF. In addition, $\Gamma$ will also potentially introduce a sign problem (because it is also a complex quantity) if the system contains many electronic states, or the TCF has a large $N$. The numerical exploration suggests that this is not a severe problem for a two-state system ${ }^{57-59}$ with a finite $N$ (for $N \leq 16$ ).

To eliminate the phase $\theta_{M}$, one can perform the following transformation ${ }^{73}$ in $\mathcal{P}$

$$
\overline{\mathcal{P}}_{n}=\mathcal{P}_{n}-i m \tilde{\omega}_{n} \mathcal{Q}_{-n}
$$

Note that such transformation on $\mathcal{P}$ has no effect on the centroid mode $\left(\mathcal{Q}_{M}\right)$, as $\tilde{\omega}_{n}$ for the centroid is zero (see Eq. 35 when $N=0$ ).

Applying the transformation $\mathcal{P}_{n} \rightarrow \overline{\mathcal{P}}_{n}$ on the Liouvillian $\mathcal{L}^{[M]}$ (see SI for details) in Eq. 70 leads to the following complex Liouvillian (in terms $\left\{\overline{\mathcal{P}}_{M}, \mathcal{Q}_{M}\right\}$ )

$$
\overline{\mathcal{L}}^{[M]}=\mathcal{L}_{\mathrm{RP}}^{[M]}+i \mathcal{L}_{\mathrm{I}}^{[M]}
$$

where we denote the real part of $\overline{\mathcal{L}}^{[M]}$ as the following non-adiabatic RPMD Liouvillian

$$
\begin{aligned}
\mathcal{L}_{\mathrm{RP}}^{[M]} & =\sum_{n=-(M-1) / 2}^{(M-1) / 2} \frac{\overline{\mathcal{P}}_{n}}{m} \frac{\vec{\partial}}{\partial \mathcal{Q}_{n}}-\left[m \tilde{\omega}_{n}^{2} \mathcal{Q}_{n}+\frac{\partial U_{0}^{[M]}\left(\boldsymbol{\mathcal { Q }}_{M}\right)}{\partial \mathcal{Q}_{n}}\right. \\
& \left.+\frac{\partial U_{\mathrm{e}}^{[M]}\left(\mathcal{Q}_{M}, \mathbf{q}, \mathbf{p}\right)}{\partial \mathcal{Q}_{n}}\right] \frac{\vec{\partial}}{\partial \overline{\mathcal{P}}_{n}}+\frac{1}{\hbar} \sum_{l=1}^{N}\left[\mathbf{p}_{l}^{\mathrm{T}} \mathcal{V}\left(R_{l}\left(\mathcal{Q}_{M}\right)\right) \vec{\nabla}_{\mathbf{q}_{l}}\right. \\
& \left.-\mathbf{q}_{l}^{\mathrm{T}} \mathcal{V}\left(R_{l}\left(\boldsymbol{\mathcal { Q }}_{M}\right)\right) \vec{\nabla}_{\mathbf{p}_{l}}\right]
\end{aligned}
$$

and the imaginary part of $\overline{\mathcal{L}}^{[M]}$ as

$$
\mathcal{L}_{\mathrm{I}}^{[M]}=\sum_{n=-(M-1) / 2}^{(M-1) / 2} \tilde{\omega}_{n}\left(\overline{\mathcal{P}}_{n} \frac{\partial}{\partial \overline{\mathcal{P}}_{-n}}-\mathcal{Q}_{n} \frac{\partial}{\partial \mathcal{Q}_{-n}}\right)
$$

Note that there is no mapping related derivative in the above imaginary Liouvillian $\mathcal{L}_{\mathrm{I}}^{[M]}$, and its impact on the electronic dynamics should only come from its influence on the nuclear dynamics, which in turn couples to the electronic mapping DOF via $\mathcal{L}_{\mathrm{RP}}^{[M]}$. Thus the influence from $\mathcal{L}_{\mathrm{I}}^{[M]}$ to mapping variables is indirect.

Using the above Liouvillian $\mathcal{L}^{[M]}$, as well as apply the transformation in Eq. 72 to the quantum Boltzmann operator and the phase space integral in Eq. 59, one has the following equivalent expression of the non-adiabatic Matsubara TCF (see SI for details)

$$
\begin{aligned}
& C_{A B}^{[M]}(t)=\frac{\alpha_{N} \cdot \alpha_{M}}{\mathcal{Z}_{M}}\left[\prod_{n=-(M-1) / 2}^{(M-1) / 2} \int_{a_{n}}^{b_{n}} d \overline{\mathcal{P}}_{n}\right] \int d \mathcal{Q}_{M} \\
\times & \int d \mathbf{q} \int d \mathbf{p} A\left(\mathcal{Q}_{M}\right) e^{-\beta H_{M}^{\mathrm{RP}}\left(\overline{\mathcal{P}}_{M}, \mathcal{Q}_{M}\right)} \Gamma\left(\boldsymbol{\mathcal { Q }}_{M}, \mathbf{q}, \mathbf{p}\right) \\
\times & e^{\overline{\mathcal{L}}^{[M]} t} B\left(\mathcal{Q}_{M}\right)
\end{aligned}
$$

where the original integral $\int_{-\infty}^{\infty} d \mathcal{P}_{n}$ becomes $\int_{a_{n}}^{b_{n}} d \overline{\mathcal{P}}_{n}$, with the integration limits $a_{n}=-\infty-i m \tilde{\omega}_{n} \mathcal{Q}_{-n}$ and $b_{n}=\infty-i m \tilde{\omega}_{n} \mathcal{Q}_{-n}$. Further, $\Gamma\left(\mathcal{Q}_{M}, \mathbf{q}, \mathbf{p}\right)$ is defined previously in Eq. 63 and, $H_{M}^{\mathrm{RP}}\left(\overline{\mathcal{P}}_{M}, \mathcal{Q}_{M}\right)$ is the ring polymer Hamiltonian in the Matsubara domain expressed as follows

$H_{M}^{\mathrm{RP}}\left(\overline{\mathcal{P}}_{M}, \mathcal{Q}_{M}\right)=\sum_{n=-(M-1) / 2}^{(M-1) / 2}\left[\frac{\overline{\mathcal{P}}_{n}^{2}}{2 m}+\frac{m}{2} \tilde{\omega}_{n}^{2} \mathcal{Q}_{n}^{2}\right]+U_{0}^{[M]}\left(\mathcal{Q}_{M}\right)$

Note that $C_{A B}^{[M]}(t)$ in Eq. 76 is exactly equivalent to Eq. 59, with the difference that Eq. 76 has a real Liouvillian and a complex nuclear phase, whereas Eq. 76 has a complex Liouvillian, and a shifted nuclear momentum in the complex plane.

\section{Exact Quantum Statistics with Matsubara Modes}

Eq. 76 is perhaps even more difficult to evaluate than Eq. 59 through a trajectory based approach, due to the 
complex phase space integral and the complex Liouvillian. However, at $t=0$, one can analytically perform the integration in the complex phase space. To this end, we use the standard contour integration procedure described by Hele et al. ${ }^{73}$, and shift each imaginary

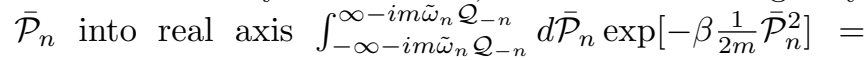
$\int_{-\infty}^{\infty} d \overline{\mathcal{P}}_{n} \exp \left[-\beta \frac{1}{2 m} \overline{\mathcal{P}}_{n}^{2}\right]$ without changing the integration. The details are discussed in the Supporting Information. This procedure allows us to write $C_{\mathrm{AB}}^{[M]}(t)$ (Eq. 76) at $t=0$ as

$$
\begin{aligned}
& C_{A B}^{[M]}(0)=\frac{\alpha_{M} \cdot \alpha_{N}}{\mathcal{Z}_{M}^{\mathrm{RP}}} \int_{-\infty}^{\infty} d \overline{\mathcal{P}}_{M} \int d \boldsymbol{\mathcal { Q }}_{M} \int d \mathbf{q} \int d \mathbf{p}(78) \\
& \times A\left(\mathcal{Q}_{M}\right) B\left(\mathcal{Q}_{M}\right) \Gamma\left(\boldsymbol{\mathcal { Q }}_{M}, \mathbf{q}, \mathbf{p}\right) e^{-\beta H_{M}^{\mathrm{RP}}\left(\overline{\mathcal{P}}_{M}, \mathcal{Q}_{M}\right)}
\end{aligned}
$$

where the original complex phase space integral becomes pure real (by shifting the momentum integral from a complex axis to a pure real axis). Note that at $t=0$, hence $e^{\mathcal{L} t}=1$, the Matsubara approximation (by discarding $L^{[N-M]}$, see Eq. 53) no longer influences the value of $C_{A B}(t)$, hence $C_{A B}^{[M]}(0)$ gives the exact QBD (where the non-Matsubara modes can be analytically integrated out and do not influence QBD, as shown in Appendix B). Thus, Boltzmann operator ensures only the Matsubara modes contribute to the exact QBD. This is a well known result for path-integral in the electronically adiabatic case. $^{38,69,89-91}$

The $C_{A B}^{[M]}(0)$ expression in Eq. 78 is reminiscent of the mapping variable (MV)-RPMD partition function expression $^{57}$, with the difference that Eq. 78 is expressed in the Matsubara space. On the other hand, one can directly obtain the MV-RPMD formalism from the generalized Kubo-Transformed TCF by taking the $t \rightarrow 0$ limit of $C_{A B}^{[N]}(t)$ in Eq. 14, (and explicitly assumes that both $\hat{A}$ and $\hat{B}$ are nuclear position related operators). Under these conditions, $[\hat{B}(t)]_{N}$ in Eq. 16 becomes

$$
\begin{aligned}
{[\hat{B}(0)]_{N}=} & \frac{1}{N} \sum_{k=1}^{N} \prod_{l=1}^{N} \int d \mathbf{D}^{\prime} \int d \boldsymbol{\Delta}^{\prime} \prod_{l=1}^{N} e^{\frac{i}{\hbar} P_{l} D_{l}^{\prime}} e^{\frac{i}{\hbar} \mathbf{p}_{l} \boldsymbol{\Delta}_{l}^{\prime}} \\
& \times \prod_{l=1}^{N} \delta\left(\boldsymbol{\Delta}_{l}^{\prime}\right) \cdot \delta\left(D_{l}^{\prime}\right) \cdot B\left(R_{k}+\frac{D_{k}}{2}\right) \\
= & \frac{1}{N} \sum_{k=1}^{N} B\left(R_{k}\right) \equiv B\left(\mathbf{R}_{N}\right)
\end{aligned}
$$

whereas the thermal Boltzmann operator becomes

$$
\begin{aligned}
& {\left[e^{-\beta \hat{H}} \hat{A}\right]_{\bar{N}}=\int d \mathbf{D} \int d \boldsymbol{\Delta} \prod_{l=1}^{N} e^{\frac{i}{\hbar} P_{l} D_{l}} e^{\frac{i}{\hbar} \mathbf{p}_{l} \boldsymbol{\Delta}_{l}}} \\
& \times \prod_{l=1}^{N}\left\langle\mathbf{q}_{l-1}-\frac{1}{2} \boldsymbol{\Delta}_{l-1}, R_{l-1}-\frac{1}{2} D_{l-1}\right| \\
& \quad \hat{\mathcal{P}} e^{-\beta_{N} \hat{H}} \hat{\mathcal{P}}\left|\mathbf{q}_{l}+\frac{\boldsymbol{\Delta}_{l}}{2}, R_{l}+\frac{D_{l}}{2}\right\rangle \cdot \frac{1}{N} \sum_{k=1}^{N} A\left(R_{k}+\frac{D_{k}}{2}\right)
\end{aligned}
$$

Putting these two expressions back to the time correlation function $C_{A B}^{[N]}(t)$ (eq. 14), and use the relation that $\frac{1}{2 \pi \hbar} \int d P_{l} e^{\frac{i}{\hbar} P_{l} D_{l}}=\delta\left(D_{l}\right)$, we can explicitly integrate out the $\int d \mathbf{P}$ integrals (which are allowed when $\hat{A}$ is not a function of $\hat{P}$ ), resulting in

$$
\begin{aligned}
& C_{A B}^{[N]}(0)=\frac{\alpha_{N}^{0}}{\mathcal{Z}} \int \mathrm{d} \mathbf{R} \int \mathrm{d} \mathbf{q} \int \mathrm{d} \mathbf{p} \int \mathrm{d} \boldsymbol{\Delta} A\left(\mathbf{R}_{N}\right) B\left(\mathbf{R}_{N}\right) \\
& \times \prod_{l=1}^{N} e^{\frac{i}{\hbar} \mathbf{p}_{l} \boldsymbol{\Delta}_{l}}\left\langle\mathbf{q}_{l-1}-\frac{1}{2} \boldsymbol{\Delta}_{l-1}, R_{l-1}\left|\hat{\mathcal{P}} e^{-\beta_{N} \hat{H}} \hat{\mathcal{P}}\right| \mathbf{q}_{l}+\frac{\boldsymbol{\Delta}_{l}}{2}, R_{l}\right\rangle,
\end{aligned}
$$

where $\alpha_{N}^{0}=(2 \pi \hbar)^{-\mathcal{K} N}$.

Through standard path-integral techniques (see details in the Supporting Information), one can explicitly show that the above $C_{A B}^{[N]}(0)$ becomes

$$
\begin{aligned}
C_{A B}^{[N]}(0)= & \frac{\alpha_{N}^{0}}{\mathcal{Z}} \int \mathrm{d} \mathbf{R} \int \mathrm{d} \tilde{\mathbf{P}} \int \mathrm{d} \mathbf{q} \int \mathrm{d} \mathbf{p} A\left(\mathbf{R}_{N}\right) B\left(\mathbf{R}_{N}\right) \\
& \times \Gamma(\mathbf{R}, \mathbf{q}, \mathbf{p}) e^{-\beta H_{N}^{\mathrm{RP}}(\tilde{\mathbf{P}}, \mathbf{R})}
\end{aligned}
$$

where $\Gamma(\mathbf{R}, \mathbf{q}, \mathbf{p})=\phi \cdot e^{-\frac{\mathcal{G}_{N}}{\hbar}} \operatorname{Tr}_{\mathrm{e}}\left[\prod_{l=1}^{N}\left(\mathbf{C}_{l}-\frac{1}{2} \boldsymbol{\mathcal { I }}\right) \mathcal{M}\left(R_{l}\right)\right]$ (see Eq. 63 for detailed expressions), and $H_{N}^{\mathrm{RP}}(\tilde{\mathbf{P}}, \mathbf{R})$ is the standard ring-polymer Hamiltonian in the primitive nuclear coordinate $\left\{R_{l}\right\}$

$$
\begin{aligned}
& H_{N}^{\mathrm{RP}}(\tilde{\mathbf{P}}, \mathbf{R})=\frac{1}{N} \sum_{l=1}^{N}\left[\frac{\tilde{P}_{l}^{2}}{2 m}+\frac{m}{2 \beta_{N}^{2} \hbar^{2}}\left(R_{l}-R_{l-1}\right)^{2}+V_{0}\left(R_{l}\right)\right], \\
& =\sum_{n=-(N-1) / 2}^{(N-1) / 2}\left[\frac{\tilde{\mathcal{P}}_{n}^{2}}{2 m}+\frac{m}{2} \omega_{n}^{2} \mathcal{Q}_{n}^{2}\right]+U_{0}^{[N]}(\mathcal{Q}),
\end{aligned}
$$

where the second line is the same ring polymer Hamiltonian expressed in the normal mode representation (through the transformations in Eq. 36, and with the normal mode frequency $\omega_{n}$ in Eq. 35). Note that the $\int d \tilde{\mathbf{P}}$ integral in Eq. 82 was reintroduced from a constant (which can be re-expressed as the nuclear momentum Gaussian integral) through the standard path-integral procedure, which does not appear in the Liouvillian of $C_{A B}^{[N]}(t)$ (Eq. 28). On the other hand, the $d \overline{\mathcal{P}}_{M}$ in $C_{A B}^{[M]}(t)$ (Eq. 76) is the actual nuclear momentum integral that appears in both initial QBD and the Liouvillian.

Note that $C_{A B}^{[N]}(0)$ is exactly equivalent to the original MV-RPMD partition function expression, with a slightly different procedure of the derivation compared to those in the original work. ${ }^{57}$ Here, we show that this partition function can also be directly obtained from the linked multi-dimensional Wigner transform.

Further, $C_{A B}^{[N]}(0)$ in Eq. 82 and $C_{A B}^{[M]}(0)$ give the same exact quantum statistics,

$$
\lim _{N \rightarrow \infty} C_{A B}^{[N]}(0)=\lim _{\substack{N \rightarrow \infty \\ M \rightarrow \infty}} \lim _{M \ll N} C_{A B}^{[M]}(0),
$$

such that the same quantum statistics can either be achieved under a large $N$ limit for regular path-integral 
ring polymer, or under the large $M$ limit for the Matsubara modes. The adiabatic limit of the above relation is a well-known result ${ }^{38,69,89-91}$. Here, we explicitly demonstrate that this is also true for the non-adiabatic scenario. Note that under the adiabatic limit, the convergence of $C_{A B}^{[N]}(0)$ with respect to an increasing $N$ is much faster $^{38,69}$ than the convergence of $C_{A B}^{[M]}(0)$ with respect to $M$ (under the $N \rightarrow \infty$ as well as $M \ll N$ limit). We have not performed any numerical test to confirm that this is also true for the non-adiabatic scenario, but we conjecture that this is the case.

\section{NON-ADIABATIC RING POLYMER MOLECULAR DYNAMICS}

The analytic continuation procedure performed in $C_{A B}^{[M]}(0)$ (Eq. 78) is not valid when $\mathrm{t}>0$ in general. This is because that when the dynamics is propagated with $\overline{\mathcal{P}}_{M}$ and $\mathcal{Q}_{M}$ in the complex plane, one may encounter well-known singularities, ${ }^{70,92}$ leading to a diverging $e^{\mathcal{L}^{[M]} t} B\left(\mathcal{Q}_{M}\right)$ such that the function of $\overline{\mathcal{P}}_{n}$ no longer approaching to 0 when $\overline{\mathcal{P}}_{n} \rightarrow \pm \infty$ from the real axis and breaks the counter integral trick (outlined in the Supporting Information).

On the other hand, as proposed in the original Matsubara dynamics work, ${ }^{73}$ it is possible to follow a path along which each $\overline{\mathcal{P}}_{n}$ is partially moved towards the real axis and $\mathcal{L}_{\mathrm{I}}^{[M]}$ is partially discarded so the contour integration trick remains valid, and at the end of the path, $\mathcal{L}_{\mathrm{I}}^{[M]}$ has been completely discarded and $\overline{\mathcal{P}}_{n}$ has reached the real axis. ${ }^{70}$ Applying this approximation on the nonadiabatic Matsubara dynamics leads to following nonadiabatic RPMD approach, which is the second key result in the paper as follows

$$
\begin{aligned}
& C_{A B}^{[M]}(t) \approx \frac{\alpha_{N} \cdot \alpha_{M}}{\mathcal{Z}_{M}^{\mathrm{RP}}} \int d \overline{\mathcal{P}}_{M} \int d \mathcal{Q}_{M} \int d \mathbf{q} \int d \mathbf{p} \\
& \times A\left(\mathcal{Q}_{M}\right) e^{-\beta H_{M}^{\mathrm{RP}}\left(\overline{\mathcal{P}}_{M}, \mathcal{Q}_{M}\right)} \Gamma\left(\mathcal{Q}_{M}, \mathbf{q}, \mathbf{p}\right) e^{\mathcal{L}_{\mathrm{RP}}^{[M]} t} B\left(\mathcal{Q}_{M}\right) .
\end{aligned}
$$

In the above NRPMD expression of TCF, the initial distribution is governed by $e^{-\beta H_{M}^{\mathrm{RP}}\left(\overline{\mathcal{P}}_{M}, \mathcal{Q}_{M}\right)} \Gamma\left(\mathcal{Q}_{M}, \mathbf{q}, \mathbf{p}\right)$, whereas the quantum dynamics is propagated by the Liouvillian $\mathcal{L}_{\mathrm{RP}}^{[M]}$ (Eq. 74).

If we choose to use ring polymer normal mode frequency instead of the Matsubara frequency in the above expression (and denote $\overline{\mathcal{P}}_{n}$ as $\mathcal{P}_{n}$ for simplicity) in Eq. 85, it then gives the non-adiabatic RPMD expression for TCF as follows

$$
\begin{aligned}
& C_{A B}^{\mathrm{NRP}}(t)=\frac{\alpha_{N} \cdot \alpha_{M}}{\mathcal{Z}_{N}^{\mathrm{RP}}} \int d \mathcal{P} \int d \mathcal{Q} \int d \mathbf{q} \int d \mathbf{p} \\
& \times \Gamma(\mathcal{Q}, \mathbf{q}, \mathbf{p}) e^{-\beta H_{N}^{\mathrm{RP}}(\mathcal{P}, \mathcal{Q})} A(\mathcal{Q}) \cdot e^{\mathcal{L}_{\mathrm{RP}}^{[N]} t} B(\boldsymbol{Q}),
\end{aligned}
$$

where $\Gamma(\mathbf{R}, \mathbf{q}, \mathbf{p})=\phi \cdot e^{-\frac{\mathcal{G}_{N}}{\hbar}} \operatorname{Tr}_{\mathrm{e}}\left[\prod_{l=1}^{N}\left(\mathbf{C}_{l}-\frac{1}{2} \boldsymbol{I}\right) \mathcal{M}\left(R_{l}\right)\right]$ (see Eq. 63 for detailed expressions), $H_{N}^{\mathrm{RP}}$ is the state-independent ring-polymer Hamiltonian (in the initial quantum Boltzmann operator) expressed as $H_{N}^{\mathrm{RP}}(\boldsymbol{P}, \mathcal{Q})=\sum_{n}\left[\frac{\mathcal{P}_{n}^{2}}{2 m}+\frac{1}{2} m \omega_{n}^{2} \mathcal{Q}_{n}^{2}\right]+U_{0}^{[N]}(\mathcal{Q})$, and the NRPMD Liouvillian is

$$
\begin{aligned}
\mathcal{L}_{\mathrm{RP}}^{[N]}= & \sum_{n=-(N-1) / 2}^{(N-1) / 2} \frac{\mathcal{P}_{n}}{m} \frac{\vec{\partial}}{\partial \mathcal{Q}_{n}}-\left[m \omega_{n}^{2} \mathcal{Q}_{n}+\frac{\partial U_{0}^{[N]}(\mathcal{Q})}{\partial \mathcal{Q}_{n}}\right. \\
& \left.+\frac{\partial U_{\mathrm{e}}^{[N]}(\boldsymbol{\mathcal { Q }}, \mathbf{q}, \mathbf{p})}{\partial \mathcal{Q}_{n}}\right] \frac{\vec{\partial}}{\partial \mathcal{P}_{n}}+\frac{1}{\hbar} \sum_{l=1}^{N}\left[\mathbf{p}_{l}^{\mathrm{T}} \mathcal{V}\left(R_{l}(\mathcal{Q})\right) \vec{\nabla}_{\mathbf{q}_{l}}\right. \\
& \left.-\mathbf{q}_{l}^{\mathrm{T}} \mathcal{V}\left(R_{l}(\mathcal{Q})\right) \vec{\nabla}_{\mathbf{p}_{l}}\right]
\end{aligned}
$$

which corresponds to the following NRPMD Hamiltonian $^{54}$ in the primitive nuclear coordinate as follows

$$
\begin{aligned}
& H_{N}^{\mathrm{NRP}}(\mathbf{P}, \mathbf{R})=\frac{1}{N} \sum_{l=1}^{N}\left[\frac{P_{l}^{2}}{2 M}+\frac{M}{2 \beta_{N}^{2} \hbar^{2}}\left(R_{l}-R_{l-1}\right)^{2}\right. \\
& \left.+V_{0}\left(R_{l}\right)+\frac{1}{2 \hbar} \sum_{i, j=1}^{\mathcal{K}} \mathcal{V}_{i j}\left(R_{l}\right)\left(\left[p_{l}\right]_{i}\left[p_{l}\right]_{j}+\left[q_{l}\right]_{i}\left[q_{l}\right]_{j}-\delta_{i j} \hbar\right)\right] .
\end{aligned}
$$

Note that the frequency $\omega_{n}$ is the ring polymer normal mode frequency (Eq. 35), whereas $\tilde{\omega}_{n}$ in Eq. 85 is the Matsubara frequency (Eq. 51).

Dropping the imaginary part of the Liouvillian $i L_{\mathrm{I}}^{[M]}$, unfortunately, introduces spurious frequency shift to the non-centroid normal modes, leading to the well-known "spurious resonances" problem in RPMD when there are resonances between ring-polymer frequencies and physical frequencies (such as stretching vibrations). ${ }^{85,93}$ This problem can be partially resolved by replacing $i L_{\mathrm{I}}^{[M]}$ by an effective white-noise FokkerPlanck operator ${ }^{94}$, leading to the Thermostatting technique for RPMD. ${ }^{85,93,94}$ This Thermostatting approach has also been recently incorporated into the NRPMD approach. ${ }^{56}$

Note that the NRPMD approach (Eq. 86) in the current work (which can be viewed as an approximation of the non-adiabatic Matsubara dynamics $C_{A B}^{[M]}$ through Eq. 85) samples the same initial distribution of MV-RPMD $\Gamma(\mathcal{Q}, \mathbf{q}, \mathbf{p}) e^{-\beta H_{N}^{\mathrm{RP}}(\mathcal{P}, \mathcal{Q})}$, whereas using the NRPMD Liouvillian in Eq. 87 (or NRPMD Hamiltonian $^{54}$ in Eq. 88) to propagate the dynamics. For a finite $N$, NRPMD does not preserve the QBD, ${ }^{54,55,95}$ due to the fact that two different effective Hamiltonians are used for the initial sampling $\left(H_{N}^{\mathrm{RP}}=\right.$ $\left.H_{N}^{\mathrm{RP}}(\boldsymbol{P}, \mathcal{Q}, \mathbf{q}, \mathbf{p})-\frac{1}{\beta} \ln \Gamma(\mathcal{Q}, \mathbf{q}, \mathbf{p})\right)$ and for the dynamical propagation $\left(H_{N}^{\mathrm{NRP}}\right.$ in Eq. 88), respectively. However, it was conjectured that under the $N \rightarrow \infty$ limit, NRPMD will preserve QBD. ${ }^{54,95}$ On the other hand, because the MMST Hamiltonian structure is preserved in the dynamics propagation, NRPMD gives the exact electronic Rabi oscillations when the nuclear dynamics is decoupled from the electronic DOF. ${ }^{54,55,78}$ This will be discussed further in Section VI as well as in Appendix C.

Further, our analytical work presented here also provides a theoretical justification for the recent numerical 
success of NRPMD, ${ }^{54,55}$ which was initially proposed as a model non-adiabatic path-integral dynamics. ${ }^{54,55}$ Note that the original version of NRPMD does not sample the MV-RPMD initial distribution $\Gamma \cdot e^{-\beta H_{N}^{\mathrm{RP}}}$ (as described in Eq. 86). Instead, it uses a simple position and momentum mapping variable resolution in the initial $\mathrm{QBD},{ }^{54}$ resulting in the original version of the NRPMD TCF ${ }^{54}$ as follows

$$
\begin{aligned}
C_{A B}^{\mathrm{NRP}^{\prime}}(t) & =\frac{1}{\mathcal{Z}_{N}^{\mathrm{RP}}} \int d \boldsymbol{P} \int d \boldsymbol{Q} \int d \mathbf{q} \int d \mathbf{p} \\
& \times \Gamma^{\prime}(\mathcal{Q}, \mathbf{q}, \mathbf{p}) e^{-\beta H_{N}^{\mathrm{RP}}(\mathcal{P}, \mathcal{Q})} A(\mathcal{Q}) \cdot e^{\mathcal{L}_{\mathrm{RP}}^{[N]} t} B(\mathcal{Q})
\end{aligned}
$$

where $\Gamma^{\prime}(\mathcal{Q}, \mathbf{q}, \mathbf{p})$ is expressed as ${ }^{54}$

$$
\begin{aligned}
& \Gamma^{\prime}(\mathcal{Q}, \mathbf{q}, \mathbf{p})=\phi^{\prime} e^{-\frac{\mathcal{G}_{N}}{\hbar}} \prod_{l=1}^{N}\left[\mathbf{p}_{l-1}^{\mathrm{T}} \mathcal{M}\left(R_{l}\right) \mathbf{q}_{l}\right] \cdot\left[\mathbf{q}_{l}^{\mathrm{T}} \mathcal{M}\left(R_{l}\right) \mathbf{p}_{l}\right] \\
& =\phi^{\prime} e^{-\frac{\mathcal{G}_{N}}{\hbar}} \prod_{l=1}^{N} \operatorname{Tr}_{\mathrm{e}}\left[\mathcal{M}\left(R_{l}\right) \mathbf{q}_{l} \mathbf{q}_{l}^{\mathrm{T}} \mathcal{M}\left(R_{l}\right) \mathbf{p}_{l} \mathbf{p}_{l}^{\mathrm{T}}\right]
\end{aligned}
$$

where $\phi^{\prime}=\left(\frac{4}{\pi^{\mathcal{K}}}\right)^{N}, \mathcal{G}_{N}=\sum_{l=1}^{N}\left(\mathbf{q}_{l} \mathbf{q}_{l}^{\mathrm{T}}+\mathbf{p}_{l} \mathbf{p}_{l}^{\mathrm{T}}\right)$, and $\mathcal{M}_{i j}\left(R_{l}\right)=\left\langle i\left|e^{-\frac{1}{2} \beta_{N} \hat{V}_{\mathrm{e}}\left(R_{l}\right)}\right| j\right\rangle$. Thus, the only difference between the original NRPMD ${ }^{54}$ and the NRPMD formalism in this work is the expression of the initial Boltzmann operator. Note that $\Gamma^{\prime}$ is pure real, as oppose to the complex $\Gamma$ in Eq. 86.

The recently developed MV-RPMD approach samples the initial distribution with $\tilde{H}_{N}^{\mathrm{RP}}=H_{N}^{\mathrm{RP}}(\mathcal{P}, \mathcal{Q}, \mathbf{q}, \mathbf{p})-$ $\frac{1}{\beta} \ln \Gamma(\mathcal{Q}, \mathbf{q}, \mathbf{p})$ (see Eq. 83), and use the same Hamiltonian to propagate dynamics, resulting in the following MV-RPMD TCF

$$
\begin{aligned}
& C_{A B}^{\mathrm{MV}}(t)=\frac{1}{\mathcal{Z}_{N}^{\mathrm{RP}}} \int d \mathcal{P} \int d \mathcal{Q} \int d \mathbf{q} \int d \mathbf{p} \\
& \times \Gamma(\mathcal{Q}, \mathbf{q}, \mathbf{p}) e^{-\beta H_{N}^{\mathrm{RP}}(\mathcal{P}, \mathcal{Q})} A(\mathcal{Q}) \cdot e^{\mathcal{L}_{\mathrm{MV}}^{[N]} t} B(\mathcal{Q}),
\end{aligned}
$$

where the MV-RPMD Liouvillian is expressed as

$$
\begin{aligned}
\mathcal{L}_{\mathrm{MV}}^{[N]}= & \sum_{n} \frac{\mathcal{P}_{n}}{m} \frac{\vec{\partial}}{\partial \mathcal{Q}_{n}}-\left[m \omega_{n}^{2} \mathcal{Q}_{n}+\frac{\partial U_{0}^{[N]}(\mathcal{Q})}{\partial \mathcal{Q}_{n}}\right. \\
& \left.-\frac{1}{\beta \Gamma} \frac{\partial \Gamma}{\partial \mathcal{Q}_{n}}\right] \frac{\vec{\partial}}{\partial \mathcal{P}_{n}}+\frac{1}{\beta \Gamma} \sum_{l=1}^{N}\left[-\left(\frac{\partial \Gamma}{\partial \mathbf{p}_{l}}\right)^{\mathrm{T}} \cdot \vec{\nabla}_{\mathbf{q}_{l}}\right. \\
& \left.+\left(\frac{\partial \Gamma}{\partial \mathbf{q}_{l}}\right)^{\mathrm{T}} \cdot \vec{\nabla}_{\mathbf{p}_{l}}\right]
\end{aligned}
$$

which directly corresponds to the Hamiltonian $\tilde{H}_{N}^{\mathrm{RP}}=$ $H_{N}^{\mathrm{RP}}(\mathcal{P}, \mathcal{Q}, \mathbf{q}, \mathbf{p})-\frac{1}{\beta} \ln \Gamma(\boldsymbol{Q}, \mathbf{q}, \mathbf{p})$. Note that in the original MV-RPMD approach, ${ }^{57}$ to facilitate the calculation with real trajectories, it was proposed to replace $\Gamma \rightarrow \Re[\Gamma]$ (only taking the real part of $\Gamma$ ) in both the initial Boltzmann distribution as well as in the above Liouvillian. This argument is based upon the fact that the partition function $\mathcal{Z}_{N}^{\mathrm{RP}}$ is real, and the operator estimators do not contain any imaginary part, hence the real and the imaginary part of the estimators are completely separated, and the ensemble average of the imaginary part should goes to zero. This is true if both $\hat{A}$ and $\hat{B}$ are not related to electronic states (mapping variables). However, there is no rigorous justification why this should also be applied to the Liouvillian. Moreover, for general operators, one should recognize that $\Gamma$ is indeed complex, and a more rigorous trajectory approach in MV-RPMD should be replacing $\Gamma \rightarrow|\Gamma|$ in the distribution and the Liouvillian, then performing the ensemble average by weighting each trajectory with phase $\Gamma /|\Gamma|$. This will be discussed in detailed in Appendix E for the NRPMD simulation which also uses the MV-RPMD initial distribution.

To the best of our knowledge, there is no rigorous theoretical justification of the Liouvillian $\mathcal{L}_{\mathrm{MV}}^{[N]}$. Because of using $\mathcal{L}_{\mathrm{MV}}^{[N]}$, MV-RPMD will not be able to provide the correct electronic Rabi oscillations when the electronic and nuclear DOFs are decoupled. In contrast, non-adiabatic Matsubara dynamics and NRPMD is exact under the electron-nuclear decoupled limit when the nuclear potential is Harmonic. On the other hand, MV-RPMD does preserve the QBD at any given $N$, because it uses the same Hamiltonian for initial sampling and for dynamics propagation. ${ }^{57}$

\section{QUANTUM DETAILED BALANCE}

We want to discuss the quantum detailed balance in our current formalism. For a system under the thermal equilibrium, the quantum expectation value does not change in time, $\langle\hat{A}(t)\rangle=\langle\hat{A}(0)\rangle$. Similarly, one can prove that

$$
C_{A B}^{\mathrm{K}}(t)=C_{B A}^{\mathrm{K}}(-t)
$$

for Kubo-transformed TCF defined in Eq. 5 the above relation is commonly referred to as the condition for satisfying detailed balance.

The detailed balance condition is also true for the generalized Kubo-transformed correlation function $C_{A B}^{[N]}(t)$ (Eq. 10), such that

$$
C_{A B}^{[N]}(t)=C_{B A}^{[N]}(-t) .
$$

This relation will also be rigorously satisfied for $C_{A B}^{[N]}(t)$ in Eq. 14 (after performing the Wigner transform and replace quantum propagator with the Liouvillian $\mathcal{L}^{[N]}$ ). Since Eq. 14 is quantum mechanically exact, the key to achieve detailed balance condition is

$$
\mathcal{L}^{[N]}\left[e^{-\beta H}\right]_{\bar{N}}=0,
$$

where $\mathcal{L}^{[N]}$ is the exact Liouvillian in Eq. 24 and $\left[e^{-\beta H}\right]_{\bar{N}}$ is the linked Wigner transformed Quantum Boltzmann Operator in Eq. 32, which is also exact quantum mechanically. 
The Matsubara partition function in Eq. 66 is expressed as

$$
\begin{aligned}
\mathcal{Z}_{M}= & \alpha_{N} \alpha_{M} \int d \mathcal{Q}_{M} \int d \mathcal{P}_{M} \int d \mathbf{q} \int d \mathbf{p} \\
& \times e^{-\beta \tilde{H}_{M}\left(\mathcal{P}_{M}, \mathcal{Q}_{M}\right)} e^{i \beta \theta_{M}\left(\mathcal{P}_{M}, \mathcal{Q}_{M}\right)}
\end{aligned}
$$

where the new effective Hamiltonian $\tilde{H}_{M}$ is expressed as $\tilde{H}_{M}\left(\mathcal{P}_{M}, \mathcal{Q}_{M}, \mathbf{q}, \mathbf{p}\right)=H_{M}\left(\boldsymbol{P}_{M}, \mathcal{Q}_{M}\right)-\frac{1}{\beta} \ln \Gamma\left(\mathcal{Q}_{M}, \mathbf{q}, \mathbf{p}\right)$ $=\sum_{n=-(M-1) / 2}^{(M-1) / 2} \frac{\mathcal{P}_{n}^{2}}{2 m}+U_{0}^{[M]}\left(\boldsymbol{\mathcal { Q }}_{M}\right)-\frac{1}{\beta} \ln \Gamma\left(\boldsymbol{\mathcal { Q }}_{M}, \mathbf{q}, \mathbf{p}\right)$.

One can prove that for the Matsubara phase $\left.\theta_{M}\left(\mathcal{P}_{M}, \mathcal{Q}_{M}\right)\right)$ is a conserved quantity of the nonadiabatic Matsubara Liouvillian $\mathcal{L}^{[M]}$ in Eq. 70, such that

$$
\begin{aligned}
\mathcal{L}^{[M]} \theta_{M}= & \sum_{n=-(M-1) / 2}^{(M-1) / 2} \frac{\mathcal{P}_{n}}{m} \frac{\partial \theta_{M}}{\partial \mathcal{Q}_{n}} \\
& -\sum_{n=-(M-1) / 2}^{(M-1) / 2}\left(\frac{\partial U_{0}^{[M]}}{\partial \mathcal{Q}_{n}}+\frac{\partial U_{\mathrm{e}}^{[M]}}{\partial \mathcal{Q}_{n}}\right) \frac{\partial \theta_{M}}{\partial \mathcal{P}_{n}} \\
& =\left(\frac{\partial U_{0}^{[M]}}{\partial \mathcal{Q}_{n}}+\frac{\partial U_{\mathrm{e}}^{[M]}}{\partial \mathcal{Q}_{n}}\right) \frac{\partial \mathcal{Q}_{n}}{\partial \tau}=0,
\end{aligned}
$$

where we have used the fact that in the Matsubara domain, $\frac{\partial U_{0}^{[M]}}{\partial \tau}=\frac{\partial U_{e}^{[M]}}{\partial \tau}=0$ for the last equality (with $\tau$ as the imaginary time) due to the cyclic symmetry. Also note that the mapping part of the Liouvillian $\left(\nabla_{\mathbf{p}_{l}}\right.$ and $\nabla_{\mathbf{q}_{l}}$ inside $\mathcal{L}^{[M]}$ does not act on $\left.\theta_{M}\left(\mathcal{P}_{M}, \mathcal{Q}_{M}\right)\right)$. The details of this proof is provided in the Supporting Information, which can be viewed as a generalization of the original proof in the adiabatic Matsubara dynamics. ${ }^{69}$

Unfortunately, we do not know whether the nonadiabatic Matsubara Liouvillian $\mathcal{L}^{[M]}$ commutes with the Hamiltonian, i.e., the validity of the the following relation

$$
\mathcal{L}^{[M]} \tilde{H}_{M}\left(\boldsymbol{P}_{M}, \boldsymbol{\mathcal { Q }}_{M}, \mathbf{q}, \mathbf{p}\right) \stackrel{?}{=} 0,
$$

thus we are not sure, at this moment, if the non-adiabatic Matsubara dynamics preserves the QBD governed by $\mathcal{Z}_{M}$.

On the other hand, when electronic and nuclear DOFs are completely decoupled, such that the Hamiltonian of the system can be written as $\hat{H}=\frac{\hat{P}^{2}}{2 m}+V_{0}(\hat{R})+$ $\sum_{i, j=1}^{\mathcal{K}} \mathcal{V}_{i j}|i\rangle\langle j|=\hat{H}_{0}+\hat{V}_{\mathrm{e}}$, the QBD is indeed preserved by $\mathcal{L}^{[M]}$ (see detailed discussions in Appendix C), because that the following relation

$$
\sum_{l=1}^{N} \frac{1}{\hbar \Gamma}\left[\mathbf{p}_{l}^{\mathrm{T}} \mathcal{V} \vec{\nabla}_{\mathbf{q}_{l}} \Gamma-\mathbf{q}_{l}^{\mathrm{T}} \mathcal{V} \vec{\nabla}_{\mathbf{p}_{l}} \Gamma(\mathbf{p}, \mathbf{q})\right]=0
$$

is satisfied by both $\mathcal{L}^{[N]}$ and $\mathcal{L}^{[M]}$, where $\Gamma(\mathbf{q}, \mathbf{p})$ no longer depends upon $\mathcal{Q}_{n}$ due to the nuclear position independent electronic potential $\hat{V}_{\mathrm{e}}=\sum_{i, j} \mathcal{V}_{i j}|i\rangle\langle j|$.
For a general case beyond this special limit, we want to explore the conditions that when non-adiabatic Matsubara dynamics preserves the QBD. By requiring Eq. 99, it leads to the following condition

$$
\begin{aligned}
& {\left[\frac{1}{\Gamma} \frac{\partial \Gamma}{\partial \mathcal{Q}_{n}}+\beta \frac{\partial U_{\mathrm{e}}^{[M]}}{\partial \mathcal{Q}_{n}}\right] \frac{\mathcal{P}_{n}}{m}} \\
& =-\sum_{l} \frac{1}{\hbar \Gamma}\left[\mathbf{p}_{l}^{\mathrm{T}} \mathcal{V}\left(R_{l}\right) \vec{\nabla}_{\mathbf{q}_{l}} \Gamma-\mathbf{q}_{l}^{\mathrm{T}} \mathcal{V}\left(R_{l}\right) \vec{\nabla}_{\mathbf{p}_{l}} \Gamma\right]
\end{aligned}
$$

where $\Gamma=\Gamma\left(\mathcal{Q}_{M}, \mathbf{q}, \mathbf{p}\right)$ in the above relation. Note that the above relation is the sufficient condition for preserving the $\mathrm{QBD}$, whereas the necessary one requires $\sum_{n}$ for all Matsubara modes in the above equation. Of course, for the electronic-nuclear decoupled case, both $\frac{\partial \Gamma}{\partial \mathcal{Q}_{n}}=0$ and $\frac{\partial \mathcal{V}}{\partial Q_{n}}=0$, as well as Eq. 100 is satisfied, hence $\mathcal{L}^{[M]}$ preserves the QBD. Beyond this special case, we do not know if Eq. 101 is always satisfied, and whether the non-adiabatic Matsubara dynamics preserves the QBD remains an open question and subject to future investigations.

Interestingly, if Eq. 101 is satisfied (i.e., the nonadiabatic Matsubara preserves QBD), then one can show that NRPMD must also preserves QBD. To explicitly demonstrate this, we rewrite the NRPMD time correlation function in Eq. 85 as follows

$$
\begin{aligned}
C_{A B}^{[M]}(t)= & \frac{\alpha_{N} \alpha_{M}}{\mathcal{Z}_{M}^{\mathrm{RP}}} \int d \overline{\boldsymbol{P}}_{M} \int d \mathcal{Q}_{M} \int d \mathbf{q} \int d \mathbf{p} \\
& \times A\left(\mathcal{Q}_{M}\right) e^{-\beta \tilde{H}_{M}^{\mathrm{RP}}\left(\overline{\mathcal{P}}_{M}, \boldsymbol{\mathcal { Q }}_{M}, \mathbf{q}, \mathbf{p}\right)} e^{\mathcal{L}_{M}^{\mathrm{RP}} t} B\left(\boldsymbol{\mathcal { Q }}_{M}\right) .
\end{aligned}
$$

where $\bar{H}_{M}^{\mathrm{RP}}\left(\overline{\mathcal{P}}_{M}, \mathcal{Q}_{M}, \mathbf{q}, \mathbf{p}\right)$ is the MV-RPMD Hamiltonian (with the Matsubara frequency instead of the ringpolymer frequency) expressed as follows

$$
\begin{aligned}
& \tilde{H}_{M}^{\mathrm{RP}}=H_{M}^{\mathrm{RP}}\left(\overline{\mathcal{P}}_{M}, \boldsymbol{\mathcal { Q }}_{M}, \mathbf{q}, \mathbf{p}\right)-\frac{1}{\beta} \ln \Gamma\left(\mathcal{Q}_{M}, \mathbf{q}, \mathbf{p}\right) \\
& =\sum_{n=-(M-1) / 2}^{(M-1) / 2}\left[\frac{\overline{\mathcal{P}}_{n}^{2}}{2 m}+\frac{m}{2} \tilde{\omega}_{n}^{2} \mathcal{Q}_{n}^{2}\right]+U_{0}^{[M]}\left(\boldsymbol{\mathcal { Q }}_{M}\right)-\frac{1}{\beta} \ln \Gamma .
\end{aligned}
$$

The condition for NRPMD to satisfy detailed balance is $\mathcal{L}_{\mathrm{RP}}^{[M]} \tilde{H}_{M}^{\mathrm{RP}}=0$, which resulting in the same condition described in Eq. 101. Hence, if non-adiabatic Matsubara dynamics preserves QBD, then so does NRPMD.

\section{TIME-CORRELATION FUNCTIONS WITH ELECTRONIC PROJECTION OPERATORS}

Beside the nuclear position auto-correlation function, the electronic projection correlation function is also an important one. ${ }^{54,55}$ For example, $\hat{A}=\hat{B}=|i\rangle\langle i|$. When $\hat{A}=|i\rangle\langle i|$, in general $\left[e^{-\beta \hat{H}} \hat{A}\right]_{\bar{N}} \neq[\hat{A}]_{N}\left[e^{-\beta \hat{H}}\right]_{\bar{N}}$ based upon Eq. 30. Hence, one needs to write down the generalized Kubo-transformed time correlation function as in Eq. 14. On the other hand, one can follow the same 
procedure to obtain the normal mode representation of $C_{A B}^{[N]}(t)$ (see Eq. 39) as

$C_{A B}^{[N]}(t)=\frac{\alpha_{N}}{\mathcal{Z}} \int \mathrm{d} \mathcal{Q} \int \mathrm{d} \mathcal{P} \int \mathrm{d} \mathbf{q} \int \mathrm{d} \mathbf{p}\left[e^{-\beta \hat{H}} \hat{A}\right]_{\bar{N}} e^{\mathcal{L}^{[N]} t}[\hat{B}]_{N}$,

as well as the exact procedure outlined in the previous section by making the Matsubara approximation (via discarding the $\mathcal{L}^{[N-M]}$, and integrating out the nonMatsubara modes), reaching to the following expression of $C_{A B}^{[M]}(t)$ (see Eq. 59 for a comparison)

$$
\begin{aligned}
C_{A B}^{[M]}(t)= & \frac{\alpha_{N} \cdot \alpha_{M}}{\mathcal{Z}_{M}} \int d \boldsymbol{\mathcal { Q }}_{M} \int d \mathcal{P}_{M} \int d \mathbf{q} \int d \mathbf{p} \\
& \times e^{-\beta\left(H_{M}\left(\mathcal{P}_{M}, \mathcal{Q}_{M}\right)-i \theta_{M}\left(\mathcal{P}_{M}, \mathcal{Q}_{M}\right)\right)} \\
& \times \Gamma_{i i}\left(\mathcal{Q}_{M}, \mathbf{q}, \mathbf{p}\right) e^{\overline{\mathcal{L}}^{[M]} t}[\hat{B}]_{N} .
\end{aligned}
$$

In the above equation, $\Gamma_{i i}$ is $\Gamma$ in Eq. 63 projected on $|i\rangle\langle i|$ as follows

$$
\begin{aligned}
& \Gamma_{i i}\left(\mathcal{Q}_{M}, \mathbf{q}, \mathbf{p}\right)=\frac{1}{N} \frac{2^{(\mathcal{K}+1) N}}{\hbar^{N}} e^{-\frac{\mathcal{G}_{N}}{\hbar}} \\
& \times \sum_{k=1}^{N} \operatorname{Tr}_{\mathrm{e}}\left[\prod_{l=1}^{k-1}\left(\mathbf{C}_{l}-\frac{1}{2} \mathcal{I}\right) \mathcal{M}_{i j}\left(R_{l}\left(\mathcal{Q}_{M}\right)\right) \times|i\rangle\langle i|\right. \\
& \left.\times \prod_{l^{\prime}=k}^{N}\left(\mathbf{C}_{l^{\prime}}-\frac{1}{2} \mathcal{I}\right) \mathcal{M}_{i j}\left(R_{l^{\prime}}\left(\mathcal{Q}_{M}\right)\right)\right] \\
& =\frac{2^{(\mathcal{K}+1) N}}{\hbar^{N}} e^{-\frac{\mathcal{G}_{N}}{\hbar}} \operatorname{Tr}_{\mathrm{e}}\left[|i\rangle\langle i| \prod_{l=1}^{N}\left(\mathbf{C}_{l}-\frac{1}{2} \mathcal{I}\right) \mathcal{M}_{i j}\left(R_{l}\left(\mathcal{Q}_{M}\right)\right)\right]
\end{aligned}
$$

where the property of trace ensures each term inside $\operatorname{Tr}_{\mathrm{e}}$ are identical, hence we can replace the bead average by the last equality. The Matsubara Liouvillian $\mathcal{L}^{[M]}$ is same as expressed in Eq. 70, and $\mathcal{Z}_{M}$ is the quantum partition function expressed in Eq. 66.

Following the same procedure of discarding the imaginary Liouvillian and shifting the momentum integral to the real axis (i.e., the Ring Polymer approximation), and replacing the Matsubara frequency with the normal mode frequency, one can arrive at the following RPMD correlation function

$$
\begin{aligned}
& C_{A B}^{\mathrm{NRP}}(t)=\frac{\alpha_{N} \cdot \alpha_{M}}{\mathcal{Z}_{N}^{\mathrm{RP}}} \int d \mathcal{P} \int d \mathcal{Q} \int d \mathbf{q} \int d \mathbf{p} \\
& \times e^{-\beta H_{N}^{\mathrm{RP}}(\mathcal{P}, \mathcal{Q})} \Gamma_{i i}(\mathcal{Q}, \mathbf{q}, \mathbf{p}) e^{\mathcal{L}_{\mathrm{RP}}^{[M]} t}[\hat{B}]_{N} .
\end{aligned}
$$

Here, for $\hat{B}=|i\rangle\langle i|$, one can use the following estimator

$$
\begin{aligned}
& {[\hat{B}]_{N}=\frac{1}{N} \sum_{k=1}^{N} \int d \boldsymbol{\Delta}_{k} e^{\frac{i}{\hbar} \mathbf{p}_{k} \boldsymbol{\Delta}_{k}}\left\langle\mathbf{q}_{k}-\frac{\boldsymbol{\Delta}_{k}}{2} \mid i\right\rangle\left\langle i \mid \mathbf{q}_{k}+\frac{\boldsymbol{\Delta}_{k}}{2}\right\rangle} \\
& =\frac{1}{N} \sum_{k=1}^{N}\left[\frac{2^{\mathcal{K}+1}}{\hbar} e^{-\frac{1}{\hbar}\left(\mathbf{q}_{k}^{2}+\mathbf{p}_{k}^{2}\right)}\left(\left[q_{k}\right]_{i}^{2}+\left[p_{k}\right]_{i}^{2}-\frac{\hbar}{2}\right)\right],(108)
\end{aligned}
$$

where the detailed proof is provided in the Supporting Information.
Alternatively, one could also use the mapping relation $|i\rangle\langle i| \rightarrow \hat{a}_{i}^{\dagger} \hat{a}_{i}$ to obtain

$$
\begin{aligned}
{[\hat{B}]_{N} } & =\frac{1}{N} \sum_{k=1}^{N} \int d \boldsymbol{\Delta}_{k} e^{\frac{i}{\hbar} \mathbf{p}_{k} \boldsymbol{\Delta}_{k}}\left\langle\mathbf{q}_{k}-\frac{\boldsymbol{\Delta}_{k}}{2}\left|\hat{a}_{i}^{\dagger} \hat{a}_{i}\right| \mathbf{q}_{k}+\frac{\boldsymbol{\Delta}_{k}}{2}\right\rangle \\
& =\frac{1}{N} \sum_{k=1}^{N} \frac{1}{2 \hbar}\left(\left[q_{k}\right]_{i}^{2}+\left[p_{k}\right]_{i}^{2}-\hbar\right) .
\end{aligned}
$$

The above estimator is used in the original NRPMD approach, ${ }^{54}$ which has been theoretically justified ${ }^{55}$ as well as in MV-RPMD approach for excited state dynamics (which has been derived based on the property of Wigner transform).$^{58}$ In fact, $C_{A B}^{[M]}(t)$ is equivalent to the NRPMD population time-correlation function, ${ }^{54,55}$ when using Eq. 109 for the estimator of the population at time $t$, with the exception that the expression of $\Gamma_{i i}$ is obtained using the Wigner representation for the mapping variable in the current theory, whereas in NRPMD, it is obtained by using a simple integral of both mapping positions and momentum. ${ }^{54}$ Our derivation explains the success of the original NRPMD approaches for simulating the population auto-correlation functions. ${ }^{54}$

\section{NON-EQUILIBRIUM TIME-CORRELATION FUNCTION}

Despite the fact that RPMD was originally developed for equilibrium quantum dynamics simulations, recent theoretical progress has demonstrated that both RPMD and CMD can provide accurate non-equilibrium dynamics upon photo-excitation. ${ }^{96}$ Thus, we conjecture that NRPMD is also capable to accurately describe the nonequilibrium TCF, and we will explicitly prove this.

For a given photo-induced process, we are often interested in the reduced density matrix dynamics upon the initial excitation of the molecular system. The reduced density matrix element can be expressed as

$$
\rho_{i j}(t)=\operatorname{Tr}\left[\hat{\rho}(0) e^{\frac{i}{\hbar} \hat{H} t}|i\rangle\langle j| e^{-\frac{i}{\hbar} \hat{H} t}\right],
$$

where the initial density operator $\hat{\rho}(0)$ is often expressed as a tensor product of the electronic and nuclear DOF as $\hat{\rho}(0)=|i\rangle\langle i| \otimes \frac{1}{\mathcal{Z}} e^{-\beta \hat{H}_{0}}$, where $\mathcal{Z}=\operatorname{Tr}\left[e^{-\beta \hat{H}_{0}}\right]$, and $\hat{H}_{0}$ is the ground state Hamiltonian

$$
\hat{H}_{0}=\frac{\hat{P}^{2}}{2 m}+U_{g}(\hat{R})
$$

with the ground state potential $U_{g}(\hat{R})$ associated with the ground electronic state $|g\rangle$.

The initial density $\hat{\rho}(0)$ is evolved under the influence of the total Hamiltonian $\hat{H}$ (Eq. 1). The reduced density matrix element can also be expressed as the following TCF

$$
\rho_{i j}(t)=C_{A B}(t)=\frac{1}{\mathcal{Z}} \operatorname{Tr}\left[e^{-\beta \hat{H}_{0}} \hat{A} e^{\frac{i}{\hbar} \hat{H} t} \hat{B} e^{-\frac{i}{\hbar} \hat{H} t}\right]
$$


where $\hat{A}=|i\rangle\langle i|$ is the initially occupied electronic state, and $\hat{B}=|i\rangle\langle j|$. Because $\hat{A}$ and $\hat{H}_{0}$ commute, $\left[\hat{A}, \hat{H}_{0}\right]=0$, hence

$$
\begin{aligned}
C_{A B}^{\mathrm{K}}(t) & =\frac{1}{\mathcal{Z} \beta} \int_{0}^{\beta} d \lambda \operatorname{Tr}\left[e^{-(\beta-\lambda) \hat{H}_{0}} \hat{A} e^{-\lambda \hat{H}_{0}} \hat{B}(t)\right] \\
& =\frac{1}{\mathcal{Z} \beta} \int_{0}^{\beta} d \lambda \operatorname{Tr}\left[e^{-(\beta-\lambda) \hat{H}_{0}} e^{-\lambda \hat{H}_{0}} \hat{A} \hat{B}(t)\right] \\
& =\frac{\int_{0}^{\beta} d \lambda}{\beta} \cdot \frac{1}{\mathcal{Z}} \operatorname{Tr}\left[e^{-\beta \hat{H}_{0}} \hat{A} \hat{B}(t)\right]=C_{A B}(t)
\end{aligned}
$$

Thus, one can rewrite the time correlation function $C_{A B}(t)$ in Eq. 112 into the Kubo-transformed timecorrelation function $C_{A B}^{\mathrm{K}}(t)$ as follows

$$
\rho_{i j}(t)=\frac{1}{\mathcal{Z} \beta} \int_{0}^{\beta} d \lambda \operatorname{Tr}\left[e^{-(\beta-\lambda) \hat{H}_{0}} \hat{A} e^{-\lambda \hat{H}_{0}} e^{\frac{i}{\hbar} \hat{H} t} \hat{B} e^{-\frac{i}{\hbar} \hat{H} t}\right] .
$$

The above TCF is not an equilibrium correlation function. Nevertheless, the Kubo-transformed structure allows us to express it as the discrete version of the timecorrelation function as in Eq. 5 .

Following exactly the same derivation we have outlined in the previous sections, we can express $C_{A B}^{\mathrm{K}}(t)$ in Eq. 113 without any approximation as follows

$$
\begin{aligned}
\rho_{i j}(t)=C_{A B}^{[N]}(t)= & \frac{\alpha_{N}}{\mathcal{Z}} \int \mathrm{d} \mathbf{R} \int \mathrm{d} \mathbf{P} \int \mathrm{d} \mathbf{q} \int \mathrm{d} \mathbf{p}( \\
& \times[\hat{A}]_{N}\left[e^{-\beta \hat{H}_{0}}\right]_{\bar{N}} e^{\mathcal{L}^{[N]} t}[\hat{B}]_{N},
\end{aligned}
$$

where the Liouvillian $\mathcal{L}^{[N]}$ has the same expression in Eq. 24, and $\left[e^{-\beta \hat{H}_{0}}\right]_{\bar{N}}$ has the same expression in Eq. 15 except that $\hat{H}$ is replaced by $\hat{H}_{0}$. There is no approximation in the above expression.

Further, to evaluate $\rho_{i j}(t),[\hat{A}]_{N}=\frac{1}{N} \sum_{k=1}^{N}\left[\hat{A}_{k}\right]_{\mathrm{W}}$ is the partial Wigner transformed projection operator (along the mapping DOF) with the following expression

$$
\begin{aligned}
& {[\hat{A}]_{N}=\frac{1}{N} \sum_{k=1}^{N} \int d \boldsymbol{\Delta}_{k} e^{\frac{i}{\hbar} \mathbf{p}_{k} \boldsymbol{\Delta}_{k}}\left\langle\mathbf{q}_{k}-\frac{\boldsymbol{\Delta}_{k}}{2} \mid i\right\rangle\left\langle i \mid \mathbf{q}_{k}+\frac{\boldsymbol{\Delta}_{k}}{2}\right\rangle} \\
& =\frac{1}{N} \sum_{k=1}^{N}\left[\frac{2^{\mathcal{K}+1}}{\hbar} e^{-\frac{1}{\hbar}\left(\mathbf{q}_{k}^{2}+\mathbf{p}_{k}^{2}\right)}\left(\left[q_{k}\right]_{i}^{2}+\left[p_{k}\right]_{i}^{2}-\frac{1}{2}\right)\right],(115
\end{aligned}
$$

where we have used the overlap relation $\left\langle\mathbf{q}_{k} \mid i\right\rangle=$ $\sqrt{\frac{2}{\hbar}} \frac{1}{(\pi \hbar)^{\mathcal{K} / 4}} q_{i} e^{\mathbf{e}_{k}^{\mathrm{T}} \mathbf{q}_{k}}$ and explicitly performing the standard Gaussian integral (see detailed derivation in Supporting Information).

Similarly, $[\hat{B}]_{N}=\frac{1}{N} \sum_{k=1}^{N}\left[\hat{B}_{k}\right]_{\mathrm{W}}$ can be expressed as

$$
\begin{aligned}
& {[\hat{B}]_{N}=\frac{1}{N} \sum_{k=1}^{N} \int d \boldsymbol{\Delta}_{k} e^{\frac{i}{\hbar} \mathbf{p}_{k} \boldsymbol{\Delta}_{k}}\left\langle\mathbf{q}_{k}-\frac{\boldsymbol{\Delta}_{k}}{2} \mid i\right\rangle\left\langle j \mid \mathbf{q}_{k}+\frac{\boldsymbol{\Delta}_{k}}{2}\right\rangle(116)} \\
& =\frac{1}{N} \sum_{k=1}^{N} \frac{2^{\mathcal{K}+1}}{\hbar} \mathcal{G} \cdot\left\{\left(\left[q_{k}\right]_{i}+i\left[p_{k}\right]_{i}\right)\left(\left[q_{k}\right]_{j}-i\left[p_{k}\right]_{j}\right)-\frac{1}{2} \delta_{i j}\right\}
\end{aligned}
$$

where $\mathcal{G}=e^{-\frac{1}{\hbar}\left(\mathbf{q}_{k}^{2}+\mathbf{p}_{k}^{2}\right)}$. On the other hand, there are other choices for the Wigner transform of operators. For example, the population can be directly Wigner transformed of $\hat{a}_{i}^{\dagger} \hat{a}_{i}$ as shown in Eq. 109, and the Wigner transform of $\hat{a}_{i}^{\dagger} \hat{a}_{j}$ as

$$
\begin{aligned}
{[\hat{B}]_{N} } & =\frac{1}{N} \sum_{k=1}^{N} \int d \boldsymbol{\Delta}_{k} e^{\frac{i}{\hbar} \mathbf{p}_{k} \boldsymbol{\Delta}_{k}}\left\langle\mathbf{q}_{k}-\frac{\boldsymbol{\Delta}_{k}}{2}\left|\hat{a}_{i}^{\dagger} \hat{a}_{j}\right| \mathbf{q}_{k}+\frac{\boldsymbol{\Delta}_{k}}{2}\right\rangle \\
& =\frac{1}{N} \sum_{k=1}^{N} \frac{1}{2 \hbar}\left(\left[q_{k}\right]_{i}\left[q_{k}\right]_{j}+\left[p_{k}\right]_{i}\left[p_{k}\right]_{j}-\hbar \delta_{i j}\right) .
\end{aligned}
$$

This estimator has been proposed in the original NRPMD work ${ }^{54,55}$. It has also been derived in the nonequilibrium TCF with MV-RPMD. ${ }^{58}$

Following the Matsubara approximation, we can derive the corresponding expression of the density matrix as follows

$$
\begin{aligned}
\rho_{i j}(t) \approx C_{A B}^{[M]}(t)= & \frac{\alpha_{N} \cdot \alpha_{M}}{\mathcal{Z}_{M}} \int d \boldsymbol{Q}_{M} \int d \boldsymbol{P}_{M} \int d \mathbf{q} \int d \mathbf{p} \\
& \times e^{-\beta\left(H_{M}-i \theta_{M}\right)}[\hat{A}]_{N} e^{\mathcal{L}^{[M]} t}[\hat{B}]_{N},(118)
\end{aligned}
$$

where the $H_{M}$ is expressed in eq. $61, \theta_{M}$ is expressed in Eq. 62, and the Liouvillian $\mathcal{L}^{[M]}$ is expressed in Eq. 70 . This is the non-adiabatic Matsubara dynamics expression for the reduced density matrix elements as the third key result of this paper.

Further making the RPMD approximation, we can derive the corresponding NRPMD expression of the reduced density matrix as the final key result of this paper

$$
\begin{aligned}
\rho_{i j}(t)_{A B}^{\mathrm{NRP}}(t)= & \frac{\alpha_{N} \cdot \alpha_{M}}{\mathcal{Z}_{N}^{\mathrm{RP}}} \int d \boldsymbol{P} \int d \boldsymbol{Q} \int d \mathbf{q} \int d \mathbf{p} \\
& \times e^{-\beta H_{N}^{\mathrm{RP}}(\mathcal{P}, \mathcal{Q})}[\hat{A}]_{N} e^{\mathcal{L}_{\mathrm{RP}}^{[N]} t}[\hat{B}]_{N},
\end{aligned}
$$

where $H_{N}^{\mathrm{RP}}(\mathcal{P}, \mathcal{Q})$ has the same expression as that in Eq. 86, and the Liouvillian $\mathcal{L}_{N}^{\mathrm{RP}}$ is expressed in Eq. 87, corresponding to the NRPMD Hamiltonian expressed in Eq. 77. This is the NRPMD expression of the reduced density matrix.

Thus, we explicitly show that NRPMD is capable to simulate non-equilibrium TCF, explaining the recent numerical success of using NRPMD to simulate the nonequilibrium population dynamics. ${ }^{56}$ Similar numerical success in simulating non-equilibrium TCF has also be achieved in MV-RPMD. ${ }^{58}$

\section{CONCLUSION AND FUTURE DIRECTIONS}

We present the non-adiabatic Matsubara dynamics, a general framework for computing the time-correlation function of electronically non-adiabatic systems. This new formalism is derived based on the generalized Kubotransformed time correlation function, using the Wigner representation for both the nuclear DOF and electronic mapping variables. ${ }^{74-76}$ By dropping the non-Matsubara 
nuclear normal modes in the quantum Liouvillian, we derive the non-adiabatic Matsubara dynamics, which can be viewed as a generalization of the original (electronically adiabatic) Matsubara dynamics. ${ }^{69}$ The nonadiabatic Matsubara dynamics has two complex phases, one from the nuclear DOF and the other from the electronic DOF. By making a nuclear momentum transformation, one can derive an equivalent expression of nonadiabatic Matsubara dynamics, which has a complex Liouvillian and a complex momentum distribution. Further making an approximation that drop the imaginary part of the Liouvillian, we arrive at the non-adiabatic ringpolymer molecular dynamics formalism. Thus, NRPMD can be viewed as an approximation of non-adiabatic Matsubara dynamics. Interestingly, the initial distribution of NRPMD coincides with that in the Mapping-Variable (MV)-RPMD ${ }^{57}$, whereas the NRPMD Liouvillian coincides with the Liouvillian used in the originally proposed $\mathrm{NRPMD}^{54}$ (which has a different initial quantum distribution). Our theoretical derivations explain the numerical success of both of these previous approaches. ${ }^{54,57} \mathrm{We}$ have further proven that the NRPMD is capable to simulate non-equilibrium TCF, hence justifies such simulations and explains the recent numerical success. ${ }^{56}$ At this moment, we are not sure whether non-adiabatic Matsubara dynamics preserves the quantum Boltzmann distribution (QBD), except for the special limit when the electronic and nuclear DOFs are completely decoupled. Nevertheless, we derived the condition under which the QBD will be preserved by non-adiabatic Matsubara dynamics. Interestingly, if non-adiabatic Matsubara dynamics preserves the QBD, then NRPMD is also guaranteed to preserve the QBD.

Let us also give more technical summaries of these equations. In this work, we started from an exact formalism of generalized Kubo-transformed TCF $C_{A B}^{[N]}(t)$ (Eq. 28). Making the Matsubara approximation by dropping the $\mathcal{L}^{[N-M]}$ as well as integrate out the nonMatsubara related terms, we have the non-adiabatic Matsubara TCF $C_{A B}^{[M]}(t)$ (Eq. 59 or equivalently, Eq. 76). Note that the exact non-adiabatic QBD is completely governed by these Matsubara modes, and completely irrelevant to the non-Matsubara modes. Further, In the Matsubara domain, $\hbar$ is effectively scaled as small as one desired (Eq. 67 and Eq. 68), such that the Liouvillian can be truncated to the linear order of the nuclear derivative $\hat{\Lambda}$ without making any approximation. This is in contrast to the Linearized path-integral approaches ${ }^{16-23}$ that often need to truncate the Liouvillian up to a certain order of $\hbar$. The Matsubara formalism, on the other hand, keep all orders of $\hbar$ within the Matsubara modes and discard all non-Matsubara modes. ${ }^{69,70}$

Further making the approximation that drops $i \mathcal{L}_{\mathrm{I}}^{[M]}$ while pushes the $\int d \overline{\mathcal{P}}_{n}$ integral to the real axis, and replace the Matsubara frequency with the ring polymer normal mode frequency, one arrived at the NRPMD TCF $C_{A B}^{\mathrm{NRP}}(t)(\mathrm{Eq} .86)$. The above connections are summa- rized as follows

$$
C_{A B}^{[N]}(t) \nrightarrow C_{A B}^{[M]}(t) \nrightarrow C_{A B}^{\mathrm{NRP}}(t)
$$

Note that both the Matsubara approximation (by dropping $\mathcal{L}^{[N-M]}$ in Eq. 131-133) and the ring polymer approximation (by dropping $i \mathcal{L}_{\mathrm{I}}^{[M]}$ in Eq. 75 ) are only related to the nuclear DOF, as these dropped term only contain the nuclear derivatives. For non-adiabatic Matsubara dynamics and NRPMD, we have not make any direct approximations in the mapping DOF. Thus, we only expect an indirect influence of these error terms in Liouvillian on the electronic mapping dynamics.

There are several interesting limits we would like to discuss as well. (i) Under the limit that the system only contains electronic subsystems, $C_{A B}^{[M]}(t)$ and $C_{A B}^{\mathrm{NRP}}(t)$ reduces to the same form of $C_{A B}^{[N]}(t)$ (note that there are still $N$ copies of the mapping DOF for all formalisms), which are all quantum mechanically exact. Hence, for isolated electronic subsystem, both non-adiabatic Matsubara dynamics and NRPMD preserves the exact quantum Rabi oscillations (where an explicit proof can be found in Ref. 78). (ii) Under the single electronic state limit (adiabatic limit), the non-adiabatic Matsubara formalism reduced to the original Matsubara dynamics. ${ }^{69,70}$ (iii) Under the decoupled limit of the electronic and nuclear DOF, non-adiabatic Matsubara dynamics rigorously preserves QBD, and gives the exact dynamics for the electronic subsystem, while only give an approximate dynamics for the nuclear subsystem (exact when the potential $V_{0}$ is purely harmonic. ${ }^{69,70}$ (iv) Under the $N=1$ limit, the NRPMD formalism reduced to Linearized Semi-classical Initial Value Representation (LSCIVR) approach (with a classical nuclear distribution instead of the Wigner distribution, see more explicit discussions in Appendix D).

The immediate future direction of the current work is to test the numerical performance of the non-adiabatic Matsubara dynamics for computing equilibrium and nonequilibrium TCFs. The current formalism of $C_{A B}^{[M]}(t)$ makes this a challenging task, because the electronic mapping DOFs also have $N$ copies, which are required to take the $N \rightarrow \infty$ limit. However, one does not have to use the same number of copies of the mapping DOF and nuclear DOF, and this can be accomplished through the mixed time-slicing technique ${ }^{97}$ which has been successfully implemented in a recent work of NRPMD. ${ }^{55}$ By using a finite number of the mapping resolution (Eq. 7 and Eq. 8), we expect to make the non-adiabatic Matsubara dynamics practical for system with a few nuclear DOF and a few electronic states. Another direction is using the non-adiabatic Matsubara dynamics framework to theoretically derive other existing state-dependent path-integral approaches, such as the non-adiabatic $\mathrm{CMD}^{98}$ (through a mean field approximation ${ }^{73,86}$ of the non-adiabatic Matsubara dynamics), the coherent-state 
$\mathrm{RPMD}^{63}$ approach (through a new non-adiabatic Matsubara dynamics that uses the Husimi representation for mapping variables and Wigner representation of nuclei), or the ring-polymer surface hopping approach ${ }^{64-68}$ (through the recently discovered connections between the quantum-classical Liouville equation (QCLE) and the fewest switches algorithm $\left.{ }^{99,100}\right)$. A third direction is to theoretically explore whether non-adiabatic Matsubara dynamics rigorously preserve quantum Boltzmann distribution (QBD). If so, then non-adiabatic Matsubara will be a trajectory-based approach that can correctly describe electronic Rabi oscillations and preserve the QBD, a method that is currently lacking. ${ }^{101}$

We hope that our current work provides a framework and a new paradigm for accurate non-adiabatic quantum dynamics approaches by interfacing the recent development in the field of mapping dynamics [such as new mapping representations ${ }^{102-106}$ as well as new estimators (window functions ${ }^{107,108}$ and the identity trick ${ }^{109}$ )] with the development of accurate nuclear quantum dynamics in the field of path-integral dynamics [such as the Matsubara dynamics ${ }^{69-72}$ and its approximations, including CMD,${ }^{40,73}$ RPMD,${ }^{43,44,73}$ mean-field Matsubara dynamics, ${ }^{86}$ quasi-CMD ${ }^{87}$, Planetary model ${ }^{70,88}$, etc]. It allows one to borrow recent developments from each subfield, and facilitate the merger of both sub-fields for developing more accurate non-adiabatic quantum dynamics approaches.

\section{ACKNOWLEDGEMENT}

This work was supported by the National Science Foundation CAREER Award under the Grant No. CHE1845747. P.H. appreciates the support from a Cottrell Scholar award (a program by Research Corporation for Science Advancement). We are indebted to Prof. Stuart Althorpe's help on understanding the Matsubara dynamics and its connection to the ring polymer molecular dynamics. We highly appreciate valuable discussions with Dr. Tim Hele, Dr. Michael Willatt, Dr. Pablo Videla, and Dr. Duncan Bossion.

\section{AVAILABILITY OF DATA}

The data that support the findings of this study are available from the corresponding author upon reasonable request.

\section{APPENDIX A: DERIVATION OF THE LIOUVILLIAN}

We provide the derivation of the exact Liouvillian $\mathcal{L}^{[N]}$ (Eq. 24) as well as the detailed expressions of the nonMatsubara Liouvillian $\mathcal{L}^{[N-M]}$ (Eq. 53)
We start by differentiating a time-dependent Wigner transformed of a general operator $\hat{A}$ as follows

$$
\frac{d}{d t}[\hat{A}(t)]_{\mathrm{w}}=\frac{i}{\hbar}[\hat{H}, \hat{A}(t)]_{\mathrm{w}}
$$

where $[\hat{A}(t)]_{\mathrm{W}}=\int d D e^{\frac{i}{\hbar} P D}\left\langle R-\frac{D}{2}|\hat{A}(t)| R+\frac{D}{2}\right\rangle$ is the Wigner transform of operator $\hat{A}(t)=e^{\frac{i}{\hbar} \hat{H}} \hat{A} e^{-\frac{i}{\hbar} \hat{H}}, \hat{H}=$ $\frac{\hat{P}^{2}}{2 m}+\hat{V}(\hat{R})$, with the nuclear position operator $\hat{R}$ and corresponding momentum operator $\hat{P}$. Further, $\hat{V}(\hat{R})$ is any general form of the potential energy operator.

Using the following equality of Wigner transform of two operators ${ }^{79,84}\left[\hat{O}_{1} \hat{O}_{2}\right]_{\mathrm{W}}=\left[O_{1}\right]_{\mathrm{W}} e^{-i \hat{\Lambda} \hbar / 2}\left[O_{2}\right]_{\mathrm{W}}$ (where $\hat{\Lambda}$ in general is written as Eq. 20), one can rewrite Eq. 120 as

$$
\begin{aligned}
& \frac{i}{\hbar}[\hat{H}, \hat{A}(t)]_{\mathrm{W}}=\frac{i}{\hbar}[\hat{H} \hat{A}(t)-\hat{A}(t) \hat{H}]_{\mathrm{W}} \\
& =\frac{i}{\hbar}\left([\hat{H}]_{\mathrm{W}} e^{-i \hat{\Lambda} \hbar / 2}[\hat{A}(t)]_{\mathrm{W}}-[\hat{A}(t)]_{\mathrm{W}} e^{-i \hat{\Lambda} \hbar / 2}[\hat{H}]_{\mathrm{W}}\right) \\
& =\frac{i}{\hbar}\left([\hat{H}]_{\mathrm{W}} e^{-i \hat{\Lambda} \hbar / 2}[\hat{A}(t)]_{\mathrm{W}}-[\hat{H}]_{\mathrm{W}} e^{i \hat{\Lambda} \hbar / 2}[\hat{A}(t)]_{\mathrm{W}}\right) \\
& =\frac{2}{\hbar}[\hat{H}]_{\mathrm{W}} \sin \left(\frac{\hat{\Lambda} \hbar}{2}\right)[\hat{A}(t)]_{\mathrm{W}},
\end{aligned}
$$

where in the final step of Eq. 121 we have used the fact that $e^{-i \hat{\Lambda} \hbar / 2}=\cos (\hat{\Lambda} \hbar / 2)-i \sin (\hat{\Lambda} \hbar / 2)$ and $e^{i \hat{\Lambda} \hbar / 2}=$ $\cos (\hat{\Lambda} \hbar / 2)+i \sin (\hat{\Lambda} \hbar / 2)$. Using Eq. 121 in Eq. 120 we have

$$
\frac{d}{d t}[\hat{A}(t)]_{\mathrm{W}}=\frac{i}{\hbar}[\hat{H}, \hat{A}(t)]_{\mathrm{W}}=\mathcal{L}[\hat{A}(t)]_{\mathrm{W}}
$$

where the Liouvillian is

$$
\mathcal{L}=\frac{2}{\hbar}[\hat{H}]_{\mathrm{W}} \sin \left(\frac{\hat{\Lambda} \hbar}{2}\right)
$$

For the case where $\hat{V}$ is electronically adiabatic, one can explicitly evaluate the detailed expression of the Liouvillian. We first take the Wigner transform of the Hamiltonian $^{110}$ as $[\hat{H}]_{\mathrm{W}}=\int d D e^{\frac{i}{\hbar} P D}\left\langle R-\frac{D}{2}|\hat{H}| R+\frac{D}{2}\right\rangle=$ $H(R, P)=\frac{P^{2}}{2 m}+V(R)$. With Eq. 123 and $[\hat{H}]_{\mathrm{W}}$, the Liouvillian is expressed as

$$
\begin{aligned}
\mathcal{L} & =\frac{2}{\hbar}\left[\frac{P^{2}}{2 m}+V(R)\right] \sin \left(\frac{\Lambda_{n} \hbar}{2}\right) \\
& =\frac{2}{\hbar} \frac{P^{2}}{2 m} \sin \left(\frac{\Lambda_{n} \hbar}{2}\right)+\frac{2}{\hbar} V(R) \sin \left(\frac{\Lambda_{n} \hbar}{2}\right) .
\end{aligned}
$$

Using the Taylor expansion of $\sin \left(\frac{\Lambda_{n} \hbar}{2}\right)=\frac{\Lambda_{n} \hbar}{2}-\frac{\Lambda_{n}^{3} \hbar^{3}}{8}+$ ... and systematically acting these operators on the $\frac{P^{2}}{2 m}$ and the $V(R)$ term, one reach to the well-known WignerMoyal series ${ }^{79,80,84}$ as follows

$$
\mathcal{L}=\frac{P}{m} \frac{\partial}{\partial R}-\frac{2}{\hbar} V(R) \cdot \sin \left(\frac{\hbar}{2} \frac{\partial}{\partial R} \frac{\vec{\partial}}{\partial P}\right)
$$


Next, we derive the explicit expression of the Exact Liouvillian of the non-adiabatic Hamiltonian in the generalized Kubo-transformed TCF which has the following expression

$$
\mathcal{L}^{[N]}=\sum_{l=1}^{N} \frac{2}{\hbar}\left[\hat{H}_{l}\right]_{\mathrm{W}} \cdot \sin \left(\frac{\hbar}{2} \hat{\Lambda}_{l}\right),
$$

where $\hat{\Lambda}_{l}$ is defined in Eq. 20, and $\left[\hat{H}_{l}\right]_{\mathrm{W}}$ is the Wigner transform of the MMST mapping Hamiltonian (Eq. 1) associated with the $l_{\mathrm{th}}$ bead, with the expression in Eq. 22 .

Because $\mathcal{L}^{[N]}$ contains $N$ mathematically identical terms (labeled as $l \in[1, N]$ ), one only needs to derive the expression of one term and sum them up. Below, we explicitly derive one of this term denoting as $\mathcal{L}^{[1]}$, and we drop the label $l$ for simplicity. With the operator $\hat{\Lambda}=\hat{\Lambda}^{\mathrm{e}}+\hat{\Lambda}^{\mathrm{n}}$ defined in Eq. 20, one can rewrite Eq. 126 as

$$
\begin{aligned}
& \mathcal{L}^{[1]}=\frac{2}{\hbar}[\hat{H}]_{\mathrm{W}} \cdot \sin \left(\frac{\hbar}{2} \hat{\Lambda}^{\mathrm{n}}+\frac{\hbar}{2} \hat{\Lambda}^{\mathrm{e}}\right) \\
= & \frac{2}{\hbar}[\hat{H}]_{\mathrm{W}} \cdot\left[\sin \left(\frac{\hbar}{2} \hat{\Lambda}^{\mathrm{n}}\right) \cos \left(\frac{\hbar}{2} \hat{\Lambda}^{\mathrm{e}}\right)+\cos \left(\frac{\hbar}{2} \hat{\Lambda}^{\mathrm{n}}\right) \sin \left(\frac{\hbar}{2} \hat{\Lambda}^{\mathrm{e}}\right)\right] .
\end{aligned}
$$

Explicitly expanding the terms related to the mapping derivatives as $\cos \left(\frac{\hbar}{2} \hat{\Lambda}^{\mathrm{e}}\right)=1-\frac{1}{8} \hbar^{2}\left[\hat{\Lambda}^{\mathrm{e}}\right]^{2}+\mathcal{O}\left(\left[\hat{\Lambda}^{\mathrm{e}}\right]^{4}\right)$, $\sin \left(\frac{\hbar}{2} \hat{\Lambda}^{\mathrm{e}}\right)=\frac{\hbar}{2} \Lambda^{\mathrm{e}}+\mathcal{O}\left(\left[\hat{\Lambda}^{\mathrm{e}}\right]^{3}\right)$, and note that the $V_{\mathrm{e}}(R, \mathbf{q}, \mathbf{p})$ term inside the $[\hat{H}]_{\mathrm{W}}$ contains up to the second order of p and $\mathbf{q}$ (see Eq. 23), such that $V_{\mathrm{e}}\left[\hat{\Lambda}^{\mathrm{e}}\right]^{n}=0$ for $n \geq 3$, one can rewrite Eq. 127 exactly as follows

$$
\begin{aligned}
\mathcal{L}^{[1]}= & \frac{2}{\hbar}\left[\frac{P^{2}}{2 m}+V_{0}(R)+\frac{1}{2 \hbar} \sum_{i, j=1}^{\mathcal{K}} \mathcal{V}_{i j}(R)\left(p_{i} p_{j}+q_{i} q_{j}-\delta_{i j} \hbar\right)\right] \\
& \times\left[\sin \left(\frac{\hbar}{2} \hat{\Lambda}^{\mathrm{n}}\right)\left(1-\frac{\hbar^{2}}{8}\left[\hat{\Lambda}^{\mathrm{e}}\right]^{2}\right)+\cos \left(\frac{\hbar}{2} \hat{\Lambda}^{\mathrm{n}}\right) \frac{\hbar}{2} \hat{\Lambda}^{\mathrm{e}}\right]
\end{aligned}
$$

Each term of Eq. 128 can be explicitly evaluated (see the Supporting Information for details), resulting in the exact non-adiabatic Liouvillian as follows

$$
\begin{aligned}
\mathcal{L}^{[1]}= & \frac{P}{m} \frac{\vec{\partial}}{\partial R}-\frac{2}{\hbar}\left[V_{0}(R)+V_{\mathrm{e}}(R, \mathbf{q}, \mathbf{p})\right] \sin \left(\frac{\hbar}{2} \frac{\overleftarrow{\partial}}{\partial R} \frac{\vec{\partial}}{\partial P}\right) \\
& +\frac{1}{\hbar}\left[\mathbf{p}^{\mathrm{T}} \mathcal{V}(R) \vec{\nabla}_{\mathbf{q}}-\mathbf{q}^{\mathrm{T}} \mathcal{V}(R) \vec{\nabla}_{\mathbf{p}}\right] \cos \left(\frac{\hbar}{2} \frac{\overleftarrow{\partial}}{\partial R} \frac{\vec{\partial}}{\partial P}\right)(129) \\
& +\frac{1}{4}\left[\overrightarrow{\boldsymbol{\nabla}}_{\mathbf{q}}^{\mathrm{T}} \mathcal{V}(R) \vec{\nabla}_{\mathbf{q}}+\vec{\nabla}_{\mathbf{p}}^{\mathrm{T}} \mathcal{V}(R) \vec{\nabla}_{\mathbf{p}}\right] \sin \left(\frac{\hbar}{2} \frac{\overleftarrow{\partial}}{\partial R} \frac{\vec{\partial}}{\partial P}\right)
\end{aligned}
$$

Adding a total of $N$ mathematically identical terms together (each has the same expression of the above Liouvillian $\mathcal{L}^{[1]}$, we have $\mathcal{L}^{[N]}$ expressed in Eq. 24 .

Next, we explicitly express the error term of the Liouvillian $\mathcal{L}^{[N-M]}$ when applying the Matsubara approximation. The Matsubara approximation discards $(N-M)$ Non-Matsubara modes from Eq. 43, where the nonMatsubara Liouvillian is expressed as follows

$$
\mathcal{L}^{[N-M]}=\mathcal{L}^{[N]}-\mathcal{L}^{[M]}=\mathcal{L}_{\mathrm{n}}^{[N-M]}+\mathcal{L}_{\mathrm{e}}^{[N-M]}+\mathcal{L}_{\mathrm{h}}^{[N-M]}
$$

where we have futher decomposing the non-Matsubara Liouvillian in three terms. Using the following trigonometric identities

$$
\begin{aligned}
& \sin (a+b)-\sin (a)=2 \sin \left(\frac{b}{2}\right) \cos \left(a+\frac{b}{2}\right) \\
& \cos (a+b)-\cos (a)=-2 \sin \left(a+\frac{b}{2}\right) \sin \left(\frac{b}{2}\right)
\end{aligned}
$$

we can express the non-matsubara Liouvillian as follow

$$
\begin{aligned}
& \mathcal{L}_{\mathrm{n}}^{[N-M]}=\sum_{n=(M+1) / 2}^{(N-1) / 2}\left[\frac{\mathcal{P}_{-n}}{m} \frac{\partial}{\partial \mathcal{Q}_{-n}}+\frac{\mathcal{P}_{n}}{m} \frac{\partial}{\partial \mathcal{Q}_{n}}\right] \\
& -\frac{4 N}{\hbar}\left[U_{0}^{[N]}(\mathcal{Q})+U_{e}^{[N]}(\boldsymbol{Q}, \mathbf{q}, \mathbf{p})\right] \sin \left(\frac{\hat{b}}{2}\right) \cos \left(\hat{a}+\frac{\hat{b}}{2}\right), \\
& \mathcal{L}_{\mathrm{e}}^{[N-M]}=-\frac{2}{\hbar} \sum_{l=1}^{N}\left[\mathbf{p}_{l}^{\mathrm{T}} \mathcal{V}\left(R_{l}(\mathcal{Q})\right) \vec{\nabla}_{\mathbf{q}_{l}}-\mathbf{q}_{l}^{\mathrm{T}} \mathcal{V}\left(R_{l}(\mathcal{Q})\right) \vec{\nabla}_{\mathbf{p}_{l}}\right] \\
& \times \sin \left(\hat{\mathrm{a}}+\frac{\hat{\mathrm{b}}}{2}\right) \sin \left(\frac{\hat{b}}{2}\right), \\
& \left.\mathcal{L}_{\mathrm{h}}^{[N-M]}=\frac{1}{2} \sum_{l=1}^{N}\left[\vec{\nabla}_{\mathbf{q}_{l}}^{\mathrm{T}} \mathcal{V}\left(R_{l}(\mathcal{Q})\right) \vec{\nabla}_{\mathbf{q}_{l}}+\vec{\nabla}_{\mathbf{p}_{l}}^{\mathrm{T}} \mathcal{V}\left(R_{l}(\mathcal{Q})\right) \vec{\nabla}_{\mathbf{p}_{l}}\right]\right] \\
& \times \sin \left(\frac{\hat{b}}{2}\right) \cos \left(\hat{a}+\frac{\hat{b}}{2}\right),
\end{aligned}
$$

where the operators $\hat{a}$ and $\hat{b}$ are defined as

$$
\begin{aligned}
& \hat{a}=\frac{\hbar}{2 N} \sum_{n=-(M-1) / 2}^{(M-1) / 2} \frac{\overleftarrow{\partial}}{\partial \mathcal{Q}_{n}} \frac{\vec{\partial}}{\partial \mathcal{P}_{n}} \\
& \hat{b}=\frac{\hbar}{2 N} \sum_{n=(M+1) / 2}^{(N-1) / 2} \frac{\overleftarrow{\partial}}{\partial \mathcal{Q}_{n}} \frac{\vec{\partial}}{\partial \mathcal{P}_{n}}+\frac{\overleftarrow{\partial}}{\partial \mathcal{Q}_{-n}} \frac{\vec{\partial}}{\partial \mathcal{P}_{-n}}
\end{aligned}
$$

Note that the entire $\mathcal{L}^{[N-M]}$ does not contain derivatives with respect to the electronic mapping variables. Hence, they do not have the direct influence on the electronic dynamics.

\section{APPENDIX B: QUANTUM BOLTZMANN DISTRIBUTION IN THE NON-ADIABATIC MATSUBARA DYNAMICS}

To obtain the Quantum Boltzmann Distribution function under Matsubara limit in Eq. 59, we first write down the total Boltzmann factor in normal mode representation as

$$
\begin{aligned}
& {\left[e^{-\beta \hat{H}}\right]_{\bar{N}}=\int d \mathcal{D} \int d \boldsymbol{\Delta} \prod_{n=-(N-1) / 2}^{(N-1) / 2} e^{\frac{i}{\hbar} N \mathcal{P}_{n} \mathcal{D}_{n}} \prod_{l=1}^{N} e^{\frac{i}{\hbar} p_{l} \Delta_{l}}} \\
& \times\left\langle\mathbf{q}_{l-1}-\frac{1}{2} \boldsymbol{\Delta}_{l-1}, \xi_{l-1}^{-}\left|\hat{\mathcal{P}} e^{-\beta_{N} \hat{H}} \hat{\mathcal{P}}\right| \mathbf{q}_{l}+\frac{\boldsymbol{\Delta}_{l}}{2}, \xi_{l}^{+}\right\rangle,
\end{aligned}
$$

which is same as Eq. 41 and $\xi^{ \pm}$is previously defined in Eq. 40. The expression in Eq. 136 is equivalent to Eq. 104 
of the work by Hele and Ananth ${ }^{78}$, but in normal mode representation.

Next, we integrate out the non-Matsubara $\mathcal{P}$. By the construction of the Matsubara dynamics, there is no functional dependency of non-Matsubara $\mathcal{P}$ modes in operator $\hat{B}$ and thus, we can integrate out the nonMatsubara $\mathcal{P}$ momenta, giving a product of Dirac delta function in non-Matsubara $\mathcal{D}$ modes of the form

$$
\begin{aligned}
& \prod_{n=-(N-1) / 2}^{-(M+1) / 2} \times \prod_{n=(M+1) / 2}^{(N-1) / 2} \int_{-\infty}^{\infty} d \mathcal{P}_{n} e^{\frac{i}{\hbar} N \mathcal{P}_{n} \mathcal{D}_{n}} \\
& =(2 \pi \hbar)^{(N-M)} \prod_{n=-(N-1) / 2}^{-(M+1) / 2} \times \prod_{n=(M+1) / 2}^{(N-1) / 2} \delta\left(\mathcal{D}_{n}\right),
\end{aligned}
$$

which helps to further integrate out the non-Matsubara $\mathcal{D}^{[N-M]}$ modes from Eq. 136. After integrating out the non-Matsubara $\mathcal{D}^{[N-M]}$ modes, $\left[e^{-\beta \hat{H}}\right]_{\bar{N}}$ (Eq. 136) becomes

$$
\begin{gathered}
{\left[e^{-\beta \hat{H}}\right]_{\bar{N}}\left(\mathcal{P}_{M}, \mathcal{Q}, \mathbf{q}, \mathbf{p}\right)=(2 \pi \hbar)^{(N-M)} \int d \mathcal{D}_{M} \int d \boldsymbol{\Delta}} \\
\times \prod_{n=-(M-1) / 2}^{(M-1) / 2} e^{i N \mathcal{P}_{n} \mathcal{D}_{n} / \hbar} \times \prod_{l=1}^{N} e^{i \mathbf{p}_{l} \boldsymbol{\Delta}_{l} / \hbar} \\
\times\left\langle\mathbf{q}_{l-1}-\frac{1}{2} \boldsymbol{\Delta}_{l-1}, \eta_{l-1}^{-}\left(\mathcal{Q}, \mathcal{D}_{M}\right)\right| \hat{\mathcal{P}} e^{-\beta_{N} \hat{H}} \hat{\mathcal{P}} \\
\times\left|\mathbf{q}_{l}+\frac{1}{2} \boldsymbol{\Delta}_{l}, \eta_{l}^{+}\left(\boldsymbol{\mathcal { Q }}, \mathcal{D}_{M}\right)\right\rangle,
\end{gathered}
$$

where $\int d \mathcal{D}_{M}=\prod_{n=-(M-1) / 2}^{n=(M-1) / 2} d \mathcal{D}_{n}$ that only includes the Matsubara modes, whereas the $\mathcal{Q}_{n}$ contains all modes (together with all $\mathcal{Q}$ dependent terms in the Liouvillian in the TCF at this moment). Further, $\eta_{l}^{ \pm}$is now expressed as

$$
\eta_{l}^{ \pm}=\sum_{n=-(N-1) / 2}^{(N-1) / 2} \sqrt{N} T_{l n} \mathcal{Q}_{n} \pm \sum_{n=-(M-1) / 2}^{(M-1) / 2} \frac{1}{2} \sqrt{N} T_{l n} \mathcal{D}_{n}
$$

noticing that $\mathcal{D}_{n}$ only contains the Matsubara modes.

We further split the Boltzmann operator in Eq. 137 through a symmetric Trotter expansion under the $N \rightarrow$ $\infty$ limit, noticing that $\left|\eta_{l}^{ \pm}\right\rangle\left\langle\eta_{l}^{ \pm}\right|$commutes with $\hat{\mathcal{P}}$ (as they belong to two different DOFs), and evaluating the nuclear kinetic energy term $\frac{\hat{P}^{2}}{2 m}$ explicitly through the standard path-integral technique (see SI for details). We can then express Eq. 137 as follows

$$
\begin{aligned}
& {\left[e^{-\beta \hat{H}}\right]_{\bar{N}}\left(\mathcal{P}_{M}, \mathcal{Q}, \mathbf{q}, \mathbf{p}\right)=(2 \pi \hbar)^{(N-M)}\left(\frac{m}{2 \pi \beta_{N} \hbar^{2}}\right)^{N / 2}(139)} \\
& \times \int d \mathcal{D}_{M} \int d \boldsymbol{\Delta} \prod_{n=-(M-1) / 2}^{(M-1) / 2} e^{\frac{i}{\hbar} N \mathcal{P}_{n} \mathcal{D}_{n}} \times \prod_{l=1}^{N} e^{\frac{i}{\hbar} \mathbf{p}_{l} \boldsymbol{\Delta}_{l}} \\
& \times \exp \left[-\beta_{N} \frac{m}{2 \beta_{N}^{2} \hbar^{2}}\left(\eta_{l-1}^{-}-\eta_{l}^{+}\right)^{2}\right] e^{-\frac{\beta_{N}}{2}\left[V_{0}\left(\eta_{l}^{-}\right)+V_{0}\left(\eta_{l}^{+}\right)\right]} \\
& \times\left\langle\mathbf{q}_{l-1}-\frac{1}{2} \boldsymbol{\Delta}_{l-1}\left|\hat{\mathcal{P}} e^{-\frac{\beta_{N}}{2}\left[\hat{V}_{\mathrm{e}}\left(\eta_{l}^{-}\right)+\hat{V}_{\mathrm{e}}\left(\eta_{l}^{+}\right)\right]} \hat{\mathcal{P}}\right| \mathbf{q}_{l}+\frac{1}{2} \boldsymbol{\Delta}_{l}\right\rangle,
\end{aligned}
$$

Applying the well known trigonometric identities, ${ }^{69,70}$ one can explicitly evaluate $\left(\eta_{l-1}^{-}-\eta_{l}^{+}\right)^{2}$ term as follows

$$
\begin{aligned}
& \sum_{n=-(M-1) / 2}^{\left(\eta_{l-1}^{-}-\eta_{l}^{+}\right)^{2}}\left(\sqrt{N-1) / 2} \mathcal{Q}_{n} \sin \xi_{n}+\sqrt{N} \frac{\mathcal{D}_{-n}}{2} \cos \xi_{n}\right)^{2} \\
& +\left(\beta_{N} \hbar\right)^{2} \sum_{n=(M+1) / 2}^{(N-1) / 2} \omega_{n}^{2} N\left(\mathcal{Q}_{n}^{2}+\mathcal{Q}_{-n}^{2}\right),
\end{aligned}
$$

where $\xi_{n}=\frac{n \pi}{N}$. Note that the first sum includes all Matsubara modes and the second sum includes all nonMatsubara modes. Using the relation in Eq. 140, the quantum Boltzmann operator in Eq. 139 becomes

$$
\begin{aligned}
& {\left[e^{-\beta \hat{H}}\right]_{\bar{N}}\left(\mathcal{P}_{M}, \mathcal{Q}, \mathbf{q}, \mathbf{p}\right)=(2 \pi \hbar)^{(N-M)}\left(\frac{m}{2 \pi \beta_{N} \hbar^{2}}\right)^{N / 2}} \\
& \times \int d \boldsymbol{D}_{M} \prod_{n=-(M-1) / 2}^{(M-1) / 2} e^{\frac{i}{\hbar} N \mathcal{P}_{n} \mathcal{D}_{n}} \\
& \times \exp \left[-\frac{m \beta_{N}}{2} \sum_{n=(M+1) / 2}^{(N-1) / 2} \omega_{n}^{2} N\left(\mathcal{Q}_{n}^{2}+\mathcal{Q}_{-n}^{2}\right)\right] \\
& \times \exp \left[-2 \beta_{N} \frac{m}{\left(\beta_{N} \hbar\right)^{2}} \times\right. \\
& \times \exp \left[-\frac{\beta_{N}}{2} \sum_{l=1}^{N}\left[\hat{V}_{0}\left(\eta_{l}^{-}\right)+\hat{V}_{0}\left(\eta_{l}^{+}\right)\right]\right] \cdot \int d \Delta \prod_{l=1}^{N} e^{\frac{i}{\hbar} \mathbf{p}_{l} \boldsymbol{\Delta}_{l}} \\
& \times\left\langle\mathbf{q}_{l-1}-\frac{1}{2} \boldsymbol{\Delta}_{l-1}\left|\hat{\mathcal{P}}^{-\frac{1}{2} \beta_{N}\left[\hat{V}_{\mathrm{e}}\left(\eta_{l}^{-}\right)+\hat{V}_{\mathrm{e}}\left(\eta_{l}^{+}\right)\right]} \hat{\mathcal{P}}\right| \mathbf{q}_{l}+\frac{1}{2} \Delta_{l}\right\rangle .
\end{aligned}
$$

Note that under the limit $N \rightarrow \infty$, the Gaussian function that involves $\mathcal{D}_{-n}$ term have the following form

$\exp \left[-\frac{m N^{2}}{2 \beta \hbar^{2}} \cos ^{2} \xi_{n} \mathcal{D}_{-n}^{2}\right]=\sqrt{\frac{2 \pi \beta \hbar^{2}}{m N^{2}}}\left[\cos \xi_{n}\right]^{-1} \cdot \delta\left(\mathcal{D}_{-n}\right)$

Using the properties that $\int f(x+a) \delta(x) d x=$ $f(a) \int \delta(x) d x$, we can move all terms related to the potential, including $\exp \left[-\beta_{N} \hat{V}_{0}\left(\eta_{l}^{ \pm}\right)\right]$and $\exp \left[-\beta_{N} \hat{V}_{\mathrm{e}}\left(\eta_{l}^{ \pm}\right)\right]$ related terms outside the $d \boldsymbol{D}_{M}$ integral (and set $D_{-n}=0$ for $\eta^{ \pm}$terms in Eq 138), resulting in

$$
\begin{aligned}
& {\left[e^{-\beta \hat{H}}\right]_{\bar{N}}\left(\mathcal{P}_{M}, \mathcal{Q}, \mathbf{q}, \mathbf{p}\right)=(2 \pi \hbar)^{(N-M)}\left(\frac{m}{2 \pi \beta_{N} \hbar^{2}}\right)^{N / 2}} \\
& \times e^{-\beta_{N} \sum_{l=1}^{N} V_{0}\left(R_{l}\right)} \exp \left[-\frac{m \beta_{N}}{2} \sum_{n=(M+1) / 2}^{(N-1) / 2} \omega_{n}^{2} N\left(\mathcal{Q}_{n}^{2}+\mathcal{Q}_{-n}^{2}\right)\right] \\
& \times \Gamma(\mathbf{q}, \mathbf{p}, \mathcal{Q}) \times \prod_{n=-(M-1) / 2}^{(M-1) / 2} \int d \mathcal{D}_{n} e^{\frac{i}{\hbar} N \mathcal{P}_{n} \mathcal{D}_{n}} \\
& \quad \times \exp \left[-N \frac{2 m}{\beta_{N} \hbar^{2}}\left(\mathcal{Q}_{-n} \sin \left(\xi_{-n}\right)+\frac{1}{2} \mathcal{D}_{n} \cos \left(\xi_{-n}\right)\right)^{2}\right] .
\end{aligned}
$$


where we have explicitly write down each integral for the Matsubara $\mathcal{D}_{n}$ mode, $R_{l}=\sum_{n=-(N-1) / 2}^{(N-1) / 2} \sqrt{N} T_{l n} \mathcal{Q}_{n}$, and we have defined the following mapping integral as

$$
\begin{aligned}
\Gamma(\mathbf{q}, \mathbf{p}, \mathcal{Q}) \equiv & \int d \boldsymbol{\Delta} \prod_{l=1}^{N} e^{\frac{i}{\hbar} \mathbf{p}_{l} \boldsymbol{\Delta}_{l}} \\
& \times\left\langle\mathbf{q}_{l-1}-\frac{1}{2} \boldsymbol{\Delta}_{l-1}\left|\hat{\mathcal{P}} e^{-\beta_{N} \hat{V}_{\mathrm{e}}\left(R_{l}\right)} \hat{\mathcal{P}}\right| \mathbf{q}_{l}+\frac{1}{2} \Delta_{l}\right\rangle
\end{aligned}
$$

We explicitly integrate out $\int d \mathcal{D}_{n}$ in the last line of Eq. 142 (by denoting $\xi_{n}=n \pi / N$ ) as follows

$$
\begin{aligned}
I_{n}=\int d \mathcal{D}_{n} \exp \left[\frac{i}{\hbar} N \mathcal{P}_{n} \mathcal{D}_{n}\right. \\
\left.\quad-N \frac{2 m}{\beta_{N} \hbar^{2}}\left(-\mathcal{Q}_{-n} \sin \xi_{n}+\frac{1}{2} \mathcal{D}_{n} \cos \xi_{n}\right)^{2}\right] \\
=\exp \left[-\frac{2 m N}{\beta_{N} \hbar^{2}} \mathcal{Q}_{-n}^{2} \sin ^{2} \xi_{n}\right] \exp \left[-N \frac{\beta_{N}}{2 m \cos ^{2} \xi_{n}} \mathcal{P}_{n}^{2}\right] \\
\times \int d \mathcal{D}_{n} \exp \left[-N \frac{m}{\beta_{N} \hbar^{2}} \frac{\cos ^{2} \xi_{n}}{2}\left(\mathcal{D}_{n}-i \frac{\beta_{N} \hbar}{m \cos ^{2} \xi_{n}} \mathcal{P}_{n}\right)^{2}\right] \\
\quad \times \exp \left[N \frac{2 m}{\beta_{N} \hbar^{2}} \sin \xi_{n} \cdot \cos \xi_{n} \cdot \mathcal{Q}_{-n} \mathcal{D}_{n}\right] .
\end{aligned}
$$

Noticing that the Gaussian term inside the above $\int d \mathcal{D}_{n}$ integral is

$$
\begin{aligned}
& \exp \left[-N \frac{m}{\beta_{N} \hbar^{2}} \frac{\cos ^{2} \xi_{n}}{2}\left(\mathcal{D}_{n}-i \frac{\beta_{N} \hbar}{m \cos ^{2} \xi_{n}} \mathcal{P}_{n}\right)^{2}\right] \\
& \sim \delta\left(\mathcal{D}_{n}-i \frac{\beta_{N} \hbar}{m \cos ^{2} \xi_{n}} \mathcal{P}_{n}\right)
\end{aligned}
$$

which again allows $d \mathcal{D}_{n}$ integral to be evaluated through steepest descent fashion, resulting in

$$
\begin{aligned}
\mathcal{I}_{n}= & \mathcal{C}_{n} \cdot \exp \left[-N \frac{\beta_{N}}{2 m \cos ^{2} \xi_{n}} \mathcal{P}_{n}^{2}+i N \frac{2 \tan \xi_{n}}{\hbar} \mathcal{Q}_{-n} \mathcal{P}_{n}\right] \\
& \times \exp \left[-\frac{2 m N}{\beta_{N} \hbar^{2}} \mathcal{Q}_{-n}^{2} \sin ^{2} \xi_{n}\right]
\end{aligned}
$$

where $C_{n}=\sqrt{\pi} / \sqrt{N \frac{m}{\beta_{N} \hbar^{2}} \frac{\cos ^{2} \xi_{n}}{2}}$.

Because the $d \mathcal{D}_{n}$ integral is in the Matsubara domain, we can further simplify the expression by taking the Matsubara limit $(N \rightarrow \infty$ and $n \in M \ll N)$, resulting in $\cos ^{2} \xi_{n} \rightarrow 1, \sin ^{2} \xi_{n} \sim \mathcal{O}\left(\left(\frac{n}{N}\right)^{2}\right) \rightarrow 0$, and $\tan \xi_{n} \rightarrow \xi_{n}=n \pi / N$. Thus, the final expression of the thermal Boltzmann operator in Eq. 142 is expressed as

$$
\left[e^{-\beta \hat{H}}\right]_{\bar{N}}\left(\boldsymbol{P}_{M}, \boldsymbol{Q}, \mathbf{q}, \mathbf{p}\right)=\left(\frac{2 \pi m}{\beta_{N}}\right)^{(N-M) / 2} e^{-\beta_{N} \sum_{l=1}^{N} V_{0}\left(R_{l}\right)}
$$$$
\times \exp \left[-\frac{m \beta_{N}}{2} \sum_{n=(M+1) / 2}^{(N-1) / 2} \omega_{n}^{2} N\left(\mathcal{Q}_{n}^{2}+\mathcal{Q}_{-n}^{2}\right)\right]
$$

$\times \Gamma(\mathbf{q}, \mathbf{p}, \mathcal{Q}) \cdot \exp \left[-\beta \frac{\mathcal{P}_{n}^{2}}{2 m}\right] \cdot \exp \left[2 i\left(\frac{n \pi}{\hbar}\right) \mathcal{Q}_{-n} \mathcal{P}_{n}\right]$

In addition, one can solve the electronic mapping integral in Eq, 143. Recall that $\hat{\mathcal{P}}=\sum_{i}|i\rangle\langle i|$ is the projection operator in SEO mapping subspace, and the overlap with electronic states is expressed as

$$
\langle\mathbf{q} \mid i\rangle=\sqrt{\frac{2}{\hbar}} \frac{1}{(\pi \hbar)^{\mathcal{K} / 4}}[\mathbf{q}]_{i} e^{-\mathbf{q}^{\mathrm{T}} \mathbf{q} / 2 \hbar}
$$

Using Eq. 148 one can re-express $\Gamma$ as follows

$$
\begin{aligned}
& \Gamma(\mathcal{Q}, \mathbf{q}, \mathbf{p})=\frac{2^{N}}{\hbar^{N}} \frac{1}{(\pi \hbar)^{N \mathcal{K} / 2}} \prod_{l=1}^{N} \int d \boldsymbol{\Delta}_{l}\left(\mathbf{q}_{l-1}-\boldsymbol{\Delta}_{l-1} / 2\right)^{\mathrm{T}} \\
& \times \mathcal{M}\left(R_{l}(\mathcal{Q})\right) \cdot\left(\mathbf{q}_{l}+\boldsymbol{\Delta}_{l} / 2\right) e^{-\frac{1}{\hbar}\left(\frac{1}{4} \boldsymbol{\Delta}_{l}^{\mathrm{T}} \boldsymbol{\Delta}_{l}+\mathbf{q}_{l}^{\mathrm{T}} \mathbf{q}_{l}-i \mathbf{p}_{l}^{\mathrm{T}} \boldsymbol{\Delta}_{l}\right)} .
\end{aligned}
$$

Rearranging the prefactors of Eq. 149 and grouping terms associated with $\Delta_{l}$ (using cyclic property of trace), we have

$$
\begin{aligned}
& \Gamma(\mathcal{Q}, \mathbf{q}, \mathbf{p})=\frac{2^{N}}{\hbar^{N}} \frac{1}{(\pi \hbar)^{N \mathcal{K} / 2}} e^{-\sum_{l=1}^{N}\left(\mathbf{q}_{l}^{\mathrm{T}} \mathbf{q}_{l}+\mathbf{p}_{l}^{\mathrm{T}} \mathbf{p}_{l}\right)} \prod_{l=1}^{N} \int d \boldsymbol{\Delta}_{l} \\
& \quad \times \operatorname{Tr}_{e}\left[\left(\mathbf{q}_{l}+\boldsymbol{\Delta}_{l} / 2\right) \cdot\left(\mathbf{q}_{l}-\boldsymbol{\Delta}_{l} / 2\right)^{\mathrm{T}} \cdot \mathcal{M}\left(R_{l}(\mathcal{Q})\right)\right] \\
& \quad \times e^{-\frac{1}{\hbar}\left(\frac{1}{2} \boldsymbol{\Delta}-i \mathbf{p}\right)^{2}}
\end{aligned}
$$

Analytically performing the integration over $\Delta_{l}$ (which is a standard gaussian integral) leads to the final form of $\Gamma(\mathcal{Q}, \mathbf{q}, \mathbf{p})$ as follows

$$
\Gamma(\mathcal{Q}, \mathbf{q}, \mathbf{p})=\phi \cdot e^{-\frac{\mathcal{G}_{N}}{\hbar}} \operatorname{Tr}_{\mathrm{e}}\left[\prod_{l=1}^{N}\left(\mathbf{C}_{l}-\frac{1}{2} \boldsymbol{\mathcal { I }}\right) \mathcal{M}\left(R_{l}(\mathcal{Q})\right)\right]
$$

where $\phi=\frac{2^{(\mathcal{K}+1) N}}{\hbar^{N}}$, with $\mathcal{G}_{N}=\sum_{l=1}^{N}\left(\mathbf{q}_{l} \mathbf{q}_{l}^{\mathrm{T}}+\mathbf{p}_{l} \mathbf{p}_{l}^{\mathrm{T}}\right)$. Further, $\mathbf{C}_{l}=\left(\mathbf{q}_{l}+i \mathbf{p}_{l}\right) \times\left(\mathbf{q}_{l}-i \mathbf{p}_{l}\right)^{\mathrm{T}}$, and $\mathcal{I}$ is the $(\mathcal{K} \times \mathcal{K})$ identity matrix. Note that $\mathbf{C}_{l}-\frac{1}{2} \mathcal{I}$ can be interpreted as the reduced density matrix associated with the $l_{\text {th }}$ bead. In addition, $\mathcal{M}_{i j}\left(R_{l}(\mathcal{Q})\right)$ is the matrix of electronic Boltzmann operator as follows $\mathcal{M}_{i j}\left(R_{l}(\mathcal{Q})\right)=$ $\left\langle i\left|e^{-\beta_{N} \hat{V}_{e}\left(R_{l}(\mathcal{Q})\right)}\right| j\right\rangle$, where $\hat{V}_{e}\left(R_{l}(\mathcal{Q})\right)$ is the state dependent potential operator.

Using the above results, we have the following thermalBoltzmann operator

$$
\begin{aligned}
& {\left[e^{-\beta \hat{H}}\right]_{\bar{N}}\left(\boldsymbol{P}_{M}, \boldsymbol{Q}, \mathbf{q}, \mathbf{p}\right)=\left(\frac{2 \pi m}{\beta_{N}}\right)^{(N-M) / 2}} \\
& \times \Gamma(\mathcal{Q}, \mathbf{q}, \mathbf{p}) \cdot \prod_{n=-(M-1) / 2} e^{-\beta \frac{\mathcal{P}_{n}^{2}}{2 m}} \cdot e^{i \beta \tilde{\omega}_{n} \mathcal{P}_{n} \mathcal{Q}_{-n}} \\
& \times \exp \left[-\beta_{N} \sum_{l=1}^{N} V_{0}\left(\sum_{n=-(N-1) / 2}^{(N-1) / 2} \sqrt{N} T_{l n} \mathcal{Q}_{n}\right)\right] \\
& \times \exp \left[-\frac{m \beta_{N}}{2} \sum_{n=(M+1) / 2}^{(N-1) / 2} \omega_{n}^{2} N\left(\mathcal{Q}_{n}^{2}+\mathcal{Q}_{-n}^{2}\right)\right] .
\end{aligned}
$$

Substituting Eq. 152 into Eq. 58 we get,

$$
\begin{aligned}
C_{A B}^{[M]}(t) & =\lim _{N \rightarrow \infty} \frac{\alpha_{N}}{\mathcal{Z}_{N}} \int d \mathcal{Q} \int d \mathcal{P}_{M} \int d \mathbf{q} \int d \mathbf{p} \\
& \times A(\mathcal{Q})\left[e^{-\beta \hat{H}}\right]_{\bar{N}}\left(\mathcal{Q}, \mathcal{P}_{M}, \mathbf{q}, \mathbf{p}\right) e^{\mathcal{L}^{[M]} t} B(\mathcal{Q}) .
\end{aligned}
$$


Under the Matsubara limit $M \rightarrow \infty$, while $M \ll N$, one recognises that the Gaussian part of the non-Matsubara normal modes in $\left[e^{-\beta \hat{H}}\right]_{\bar{N}}\left(\boldsymbol{P}_{M}, \boldsymbol{Q}, \mathbf{q}, \mathbf{p}\right)$ are nascent Dirac delta functions (by noticing the expression of $\omega_{n}$ in Eq. 35) as follows

$$
\lim _{\substack{M \rightarrow \infty \\ M \ll N}} \exp \left(-\frac{2 m N^{2} \sin ^{2}(n \pi / N) \mathcal{Q}_{n}^{2}}{\beta \hbar^{2}}\right) \sim \delta\left(\mathcal{Q}_{n}\right) .
$$

Thus, we can further integrate out the non-Matsubara $\mathcal{Q}$ modes from Eq. 152 (based on a steepest descent argument), leading to the following effective changes inside the nuclear coordinate integral

$$
\begin{aligned}
& \exp \left[-\beta_{N} \sum_{l=1}^{N} V_{0}\left(\sum_{n=-(N-1) / 2}^{(N-1) / 2} T_{l n} \mathcal{Q}_{n}\right)\right] \\
\rightarrow & \exp \left[-\beta U_{0}^{[M]}\left(\mathcal{Q}_{M}\right)\right]
\end{aligned}
$$

where $U_{0}^{[M]}\left(\mathcal{Q}_{M}\right)$ is defined in Eq. 61 , as well as

$\operatorname{Tr}_{\mathrm{e}}\left[\prod_{l=1}^{N}\left(\mathcal{C}_{l}-\frac{1}{2} \mathcal{I}\right) \mathcal{M}_{i j}(\mathcal{Q})\right] \rightarrow \operatorname{Tr}_{\mathrm{e}}\left[\prod_{l=1}^{N}\left(\mathcal{C}_{l}-\frac{1}{2} \mathcal{I}\right) \mathcal{M}_{i j}\left(\mathcal{Q}_{M}\right)\right]$

for the $\Gamma$ related term. Because these quantities only depend on Matsubara mode $\mathcal{Q}_{M}$, we can move them outside the integral of the non-Matsubara modes

$$
\begin{aligned}
& C_{A B}^{[M]}(t)=\lim _{N \rightarrow \infty} \frac{\alpha_{N}}{\mathcal{Z}_{N}} \int d \mathcal{Q}_{M} \int d \mathcal{P}_{M} \int d \mathbf{q} \int d \mathbf{p} \\
& \times A\left(\mathcal{Q}_{M}\right)\left[e^{-\beta \hat{H}}\right]_{\bar{N}}\left(\mathcal{Q}_{M}, \mathcal{P}_{M}, \mathbf{q}, \mathbf{p}\right) e^{\mathcal{L}^{[M]} t} B\left(\mathcal{Q}_{M}\right) \\
& \times \prod_{n=(M+1) / 2}^{(N-1) / 2} \int d \mathcal{Q}_{n} d \mathcal{Q}_{-n} e^{-\beta \sum_{n=(M+1) / 2}^{(N-1) / 2} \frac{m}{2} \omega_{n}^{2}\left(\mathcal{Q}_{n}^{2}+\mathcal{Q}_{-n}^{2}\right),}
\end{aligned}
$$

where Gaussian integral in the last line of the above equation can be analytically performed, resulting in the constant $\alpha_{M}$ in Eq. 60. The final results of $C_{A B}^{[M]}(t)$ is expressed in Eq. 59.

\section{APPENDIX C: DETAILED BALANCE FOR SYSTEM WITH DECOUPLED ELECTRONIC AND NUCLEAR DOF}

For the system that has a decoupled electronic-nuclear interaction,

$$
\hat{H}=\frac{\hat{P}^{2}}{2 m}+V_{0}(\hat{R})+\sum_{i, j=1}^{\mathcal{K}} \mathcal{V}_{i j}|i\rangle\langle j|=\hat{H}_{0}+\hat{V}_{\mathrm{e}}
$$

where $\mathcal{V}_{i j}$ is a constant thus $\partial V_{i j} / \partial R=0$. This case also include two limits (1) electronically adiabatic system $\left(\hat{V}_{e}=0\right)$ or $(2)$ there is only electronic subsystem $(\hat{T}+$ $\left.\hat{V}_{0}=0\right)$.
For the electronic-nuclear decoupling case, the exact thermal Boltzmann operator is

$$
\begin{aligned}
{\left[e^{-\beta \hat{H}}\right]_{\bar{N}}=} & \Gamma(\mathbf{q}, \mathbf{p}) \int d \mathbf{D} \prod_{l=1}^{N} e^{\frac{i}{\hbar} P_{l} D_{l}} \\
& \times\left\langle R_{l-1}-\frac{1}{2} D_{l-1}\left|e^{-\beta_{N} \hat{H}_{0}}\right| R_{l}+\frac{1}{2} D_{l}\right\rangle
\end{aligned}
$$

where the electronic part $\Gamma(\mathbf{q}, \mathbf{p})$ becomes

$$
\Gamma(\mathbf{q}, \mathbf{p})=\frac{2^{(\mathcal{K}+1) N}}{\hbar^{N}} e^{-\frac{\mathcal{G}_{N}}{\hbar}} \operatorname{Tr}_{\mathrm{e}}\left[\prod_{l=1}^{N}\left(\mathbf{C}_{l}-\frac{1}{2} \mathcal{I}\right) \mathcal{M}\right]
$$

with $\mathcal{G}_{N}=\sum_{l=1}^{N}\left(\mathbf{q}_{l} \mathbf{q}_{l}^{\mathrm{T}}+\mathbf{p}_{l} \mathbf{p}_{l}^{\mathrm{T}}\right)$. Further, $\mathbf{C}_{l}=\left(\mathbf{q}_{l}+\right.$ $\left.i \mathbf{p}_{l}\right) \times\left(\mathbf{q}_{l}-i \mathbf{p}_{l}\right)^{\mathrm{T}}, \mathcal{I}$ is the $(\mathcal{K} \times \mathcal{K})$ identity matrix and $\mathcal{M}_{i j}=\left\langle i\left|e^{-\beta_{N} \hat{V}_{e}}\right| j\right\rangle$.

The exact Liouvillian in Eq. 24 becomes

$$
\begin{aligned}
\mathcal{L}^{[N]}= & \sum_{l=1}^{N}\left\{\frac{P_{l}}{m} \frac{\vec{\partial}}{\partial R_{l}}-V_{0}\left(R_{l}\right) \frac{2}{\hbar} \sin \left(\frac{\hbar}{2} \frac{\overleftarrow{\partial}}{\partial R_{l}} \frac{\vec{\partial}}{\partial P_{l}}\right)(1\right. \\
& \left.+\frac{1}{\hbar}\left[\mathbf{p}_{l}^{\mathrm{T}} \mathcal{V} \vec{\nabla}_{\mathbf{q}_{l}}-\mathbf{q}_{l}^{\mathrm{T}} \mathcal{V} \vec{\nabla}_{\left.\mathbf{p}_{l}\right]}\right]\right\}
\end{aligned}
$$

where the nuclear Liouvillian (the first term) and the electronic Liouvillian (the second term) are completely decoupled. The detailed balance condition $\mathcal{L}^{[N]}\left[e^{\beta \hat{H}}\right]_{\bar{N}}=$ 0 leads to

$$
\sum_{l=1}^{N} \frac{1}{\hbar \Gamma}\left[\mathbf{p}_{l}^{\mathrm{T}} \mathcal{V} \vec{\nabla}_{\mathbf{q}_{l}} \Gamma-\mathbf{q}_{l}^{\mathrm{T}} \mathcal{V} \vec{\nabla}_{\mathbf{p}_{l}} \Gamma\right]=0
$$

for the electronic subsystem, and $\mathcal{L}^{[N]}\left\{\int d \mathbf{D} \prod_{l=1}^{N} e^{\frac{i}{\hbar} P_{l} D_{l}}\left\langle R_{l-1}-\frac{1}{2} D_{l-1}\left|e^{-\beta_{N} \hat{H}_{0}}\right| R_{l} \quad+\right.\right.$ $\left.\left.\frac{1}{2} D_{l}\right\rangle\right\}=0$ for the nuclear DOF.

Under the same decouple limit, the non-adiabatic Matsubara Liouvillian becomes

$$
\begin{aligned}
\mathcal{L}^{[M]}= & \sum_{n=-(M-1) / 2}^{(M-1) / 2} \frac{\mathcal{P}_{n}}{m} \frac{\vec{\partial}}{\partial \mathcal{Q}_{n}}-\frac{\partial U_{0}^{[M]}\left(\mathcal{Q}_{M}\right)}{\partial \mathcal{Q}_{n}} \frac{\vec{\partial}}{\partial \mathcal{P}_{n}} \\
& +\frac{1}{\hbar} \sum_{l=1}^{N}\left(\mathbf{p}_{l}^{\mathrm{T}} \mathcal{V} \vec{\nabla}_{\mathbf{q}_{l}}-\mathbf{q}_{l}^{\mathrm{T}} \mathcal{V} \vec{\nabla}_{\mathbf{p}_{l}}\right),
\end{aligned}
$$

and the Hamiltonian $\tilde{H}_{\mathrm{M}}$ becomes

$$
\tilde{H}_{M}=\sum_{n=-(M-1) / 2}^{(M-1) / 2} \frac{\mathcal{P}_{n}^{2}}{2 m}+U_{0}^{[M]}\left(\mathcal{Q}_{M}\right)-\frac{1}{\beta} \ln \Gamma(\mathbf{q}, \mathbf{p})
$$

Hence the non-adiabatic Matsubara Liouvillian becomes the separable Matsubara Liouvillian for the nuclear DOF (first line) and the mapping Liouvillian for the isolated electronic DOF (second line). Comparing with the exact Liouvillian in Eq. 161, one can see that the electronic part of the Liouvillian is also exact in $\mathcal{L}^{[M]}$ for this special case. 
Then we can show that under such decoupled limit, non-adiabatic Matsubara dynamics preserves the QBD as follows

$\mathcal{L}^{[M]} \tilde{H}_{M}\left(\mathcal{P}_{M}, \mathcal{Q}_{M}, \mathbf{q}, \mathbf{p}\right)$

$=\mathcal{L}^{[M]} H_{M}\left(\boldsymbol{P}_{M}, \mathcal{Q}_{M}\right)+\sum_{l=1}^{N} \frac{1}{\hbar \Gamma}\left[\mathbf{p}_{l}^{\mathrm{T}} \mathcal{V} \vec{\nabla}_{\mathbf{q}_{l}} \Gamma-\mathbf{q}_{l}^{\mathrm{T}} \mathcal{V} \vec{\nabla}_{\mathbf{p}_{l}} \Gamma(\mathbf{p}, \mathbf{q})\right]$

$=0$

where we have used Eq. 162, as well as $\mathcal{L}^{[M]} H_{M}\left(\mathcal{P}_{M}, \mathcal{Q}_{M}\right)=0$ (which can be easily verified by acting $\mathcal{L}^{[M]}$ on $H_{M}\left(\mathcal{P}_{M}, \mathcal{Q}_{M}\right)$. Hence, we proved that under the decoupling limit, non-adiabatic Matsubara dynamics preserves the QBD.

\section{APPENDIX D: CONNECTIONS TO LINEARIZED PATH-INTEGRAL APPROACHES}

We would like to connect the current formalism of $C_{A B}^{[N]}(t)$ with previously developed linearized pathintegral approaches. Most of these approaches are based on the Wigner representations for the mapping and nuclear DOF, which can be formally viewed as various approximate forms of $N=1$ case of $C_{A B}^{[N]}(t)$. Since they are extensively used to compute density matrix dynamics, we will mainly focus our discussion on the non-equilibrium correlation function (Eq. 112). On the other hand, the following discussions on Liouvillian are also valid for the thermal-equilibrium time-correlation function (Eq. 14).

We begin by writing down the non-equilibrium timecorrelation function in Eq. 114 with $N=1$ as follows

$$
\begin{aligned}
C_{A B}^{[1]}(t)= & \frac{\alpha_{1}}{\mathcal{Z}} \int \mathrm{d} \mathbf{R} \int \mathrm{d} \mathbf{P} \int \mathrm{d} \mathbf{q} \int \mathrm{d} \mathbf{p} \\
& \times[\hat{A}]_{\mathrm{W}}\left[e^{-\beta \hat{H}_{0}}\right]_{\mathrm{W}} e^{\mathcal{L}^{[1]} t}[\hat{B}]_{\mathrm{W}},
\end{aligned}
$$

where $[\hat{A}]_{\mathrm{W}}=\int d \boldsymbol{\Delta} e^{\frac{i}{\hbar} \mathbf{p} \boldsymbol{\Delta}}\left\langle\mathbf{q}-\frac{\boldsymbol{\Delta}}{2} \mid i\right\rangle\left\langle i \mid \mathbf{q}+\frac{\Delta}{2}\right\rangle$ and $\left[e^{-\beta \hat{H}_{0}}\right]_{\mathrm{W}}=\int d D e^{\frac{i}{\hbar} P D}\left\langle R-D / 2\left|e^{-\beta_{N} \hat{H}_{0}}\right| R+D / 2\right\rangle$, and the Liouvillian is

$$
\begin{aligned}
& \mathcal{L}^{[1]}=\frac{P}{m} \frac{\vec{\partial}}{\partial R}-\frac{2}{\hbar}\left[V_{0}(R)+V_{\mathrm{e}}(R, \mathbf{q}, \mathbf{p})\right] \sin \left(\frac{\hbar}{2} \frac{\overleftarrow{\partial}}{\partial R} \frac{\vec{\partial}}{\partial P}\right) \\
& +\left[\mathbf{p}^{\mathrm{T}} \mathcal{V}(R) \vec{\nabla}_{\mathbf{q}}-\mathbf{q}^{\mathrm{T}} \mathcal{V}(R) \vec{\nabla}_{\mathbf{p}}\right] \frac{1}{\hbar} \cos \left(\frac{\hbar}{2} \frac{\overleftarrow{\partial}}{\partial R} \frac{\vec{\partial}}{\partial P}\right) \\
& +\frac{1}{4}\left[\vec{\nabla}_{\mathbf{q}}^{\mathrm{T}} \mathcal{V}(R) \vec{\nabla}_{\mathbf{q}}+\vec{\nabla}_{\mathbf{p}_{l}}^{\mathrm{T}} \mathcal{V}(R) \vec{\nabla}_{\mathbf{p}}\right] \sin \left(\frac{\hbar}{2} \frac{\overleftarrow{\partial}}{\partial R} \frac{\vec{\partial}}{\partial P}\right)
\end{aligned}
$$

The expressions of $C_{A B}^{[1]}(t)$ and the above Liouvillian $\mathcal{L}^{[1]}$ are in principle exact, which give rise to the exact quantum dynamics. Note that the mapping variables $q_{i}, p_{i}$ are in the order of $\mathcal{O}(\sqrt{\hbar})$ (because $\left[p_{i}, q_{i}\right]=i \hbar$, hence there is an $1 / 2 \hbar$ term in the MMST Hamiltonian in Eq. 4).

Of course, one can make approximations to $\mathcal{L}^{[1]}$. For example, if one truncate all terms up to $\mathcal{O}(\hbar)$ as follows

$$
\begin{aligned}
& \frac{2}{\hbar} \sin \left(\frac{\hbar}{2} \frac{\overleftarrow{\partial}}{\partial R} \frac{\vec{\partial}}{\partial P}\right) \approx \frac{\overleftarrow{\partial}}{\partial R} \frac{\vec{\partial}}{\partial P}+\mathcal{O}\left(\hbar^{2}\right) \\
& \frac{1}{\hbar} \cos \left(\frac{\hbar}{2} \frac{\overleftarrow{\partial}}{\partial R} \frac{\vec{\partial}}{\partial P}\right) \approx \frac{1}{\hbar}+\mathcal{O}(\hbar)
\end{aligned}
$$

and drop the third line in $\mathcal{L}^{[1]}$ which corresponds to a term that is in the order of $\mathcal{O}\left(\hbar^{0}\right)$, the Liouvillian $\mathcal{L}^{[1]}$ is reduced to the following form

$$
\begin{aligned}
\mathcal{L}_{\mathrm{LSC}}^{[1]}= & \frac{P}{m} \frac{\vec{\partial}}{\partial R}-\frac{2}{\hbar}\left[V_{0}(R)+V_{\mathrm{e}}(R, \mathbf{q}, \mathbf{p})\right] \frac{\overleftarrow{\partial}}{\partial R} \frac{\vec{\partial}}{\partial P} \\
& +\frac{1}{\hbar}\left[\mathbf{p}^{\mathrm{T}} \mathcal{V}(R) \vec{\nabla}_{\mathbf{q}}-\mathbf{q}^{\mathrm{T}} \mathcal{V}(R) \vec{\nabla}_{\mathbf{p}}\right]+\mathcal{O}\left(\hbar^{0}\right)
\end{aligned}
$$

which is the Liouvillian used in the Linearized Semiclassical Initial Value Representation (LSC-IVR) approach. Note that the error for the first line in $\mathcal{L}_{\mathrm{LSC}}$ is $\mathcal{O}\left(\hbar^{2}\right)$, and the error for the second line in $\mathcal{L}_{\mathrm{LSC}}$ is $\mathcal{O}(\hbar)$. However, the dropped term (the third line) in $\mathcal{L}^{[1]}$ corresponds to a term of $\mathcal{O}\left(\hbar^{0}\right)$. This ultimately determines the accuracy of $\mathcal{L}_{\mathrm{LSC}}^{[1]}$ to be up to $\mathcal{O}\left(\hbar^{0}\right)$.

On the other hand, if one choose to truncate $\mathcal{L}^{[1]}$ up to the linear order of nuclear operator $\hat{\Lambda}^{\mathrm{n}}=\frac{\overleftarrow{\partial}}{\partial R} \frac{\vec{\partial}}{\partial P}$, which is commonly referred to the mixed quantum-classical (MQC) Liouville approximation, ${ }^{111}$ then $\mathcal{L}^{[1]}$ reduces to the following

$$
\begin{aligned}
\mathcal{L}_{\mathrm{MQC}}^{[1]}= & \frac{P}{m} \frac{\partial}{\partial R}-\frac{2}{\hbar}\left[V_{0}(R)+V_{\mathrm{e}}(R, \mathbf{q}, \mathbf{p})\right] \frac{\overleftarrow{\partial}}{\partial R} \frac{\vec{\partial}}{\partial P} \\
& +\frac{1}{\hbar}\left[\mathbf{p}^{\mathrm{T}} \mathcal{V}(R) \vec{\nabla}_{\mathbf{q}}-\mathbf{q}^{\mathrm{T}} \mathcal{V}(R) \overrightarrow{\boldsymbol{\nabla}}_{\mathbf{p}}\right] \\
& +\frac{\hbar}{8}\left[\overrightarrow{\boldsymbol{\nabla}}_{\mathbf{q}}^{\mathrm{T}} \mathcal{V}(R) \vec{\nabla}_{\mathbf{q}}+\vec{\nabla}_{\mathbf{p}_{l}}^{\mathrm{T}} \mathcal{V}(R) \vec{\nabla}_{\mathbf{p}}\right] \frac{\grave{\partial}}{\partial R} \frac{\vec{\partial}}{\partial P}+\mathcal{O}\left(\hat{\Lambda}_{\mathrm{n}}^{2}\right)
\end{aligned}
$$

which was first derived in the Possion Bracket Mapping Equation (PBME) approach. ${ }^{25,81}$ Note that the last term scales as $\mathcal{O}\left(\hbar^{0}\right)$. Hence the accuracy of $\mathcal{L}_{\text {MQC }}^{[1]}$ is in principle up to $\mathcal{O}(\hbar)$. However, the last term was not straightforward to evaluate. ${ }^{81}$ Hence, in the common PBME approach this term is often dropped, and $\mathcal{L}_{\mathrm{LSC}}^{[1]}$ is used in the PBME calculation. Later, it was shown ${ }^{81,83}$ that this term can be equivalently expressed as

$$
\begin{aligned}
& \frac{\hbar}{8}\left[\vec{\nabla}_{\mathbf{q}}^{\mathrm{T}} \mathcal{V}(R) \vec{\nabla}_{\mathbf{q}}+\vec{\nabla}_{\mathbf{p}_{l}}^{\mathrm{T}} \mathcal{V}(R) \vec{\nabla}_{\mathbf{p}}\right] \frac{\overleftarrow{\partial}}{\partial R} \frac{\vec{\partial}}{\partial P} \\
& =\sum_{i j} \frac{\mathcal{K}}{2(\mathcal{K}+4) \hbar}\left(q_{j} q_{j}+p_{i} p_{j}-\frac{\hbar}{2} \delta_{i j}\right) \frac{\partial \mathcal{V}_{i j}(R)}{\partial R} \frac{\partial}{\partial P}
\end{aligned}
$$

Explicitly including this term and using $\mathcal{L}_{\mathrm{MQC}}^{[1]}$ for QCLE (which is referred to as the non-Hamiltonian $\mathrm{PBME}^{83}$ ) indeed improves the accuracy of the population dynamics in spin-boson problems. ${ }^{83}$ Note that under the Matsubara limit, this term can be make as small as needed (see Eq. 71)

If one wants to continue to improve the accuracy of the Liouvillian, the next term to include will be $\mathcal{O}(\hbar)$ 
comes from the quadratic term in the cos expansion in the second line of $\mathcal{L}^{[1]}$. Retaining this $\mathcal{O}(\hbar)$ term one can write the following non-Hamiltonian (NH) Liouvillian

$$
\begin{aligned}
\mathcal{L}_{\mathrm{NH}}^{[1]}= & \frac{P}{m} \frac{\partial}{\partial R}-\frac{2}{\hbar}\left[V_{0}(R)+V_{\mathrm{e}}(R, \mathbf{q}, \mathbf{p})\right] \frac{\overleftarrow{\partial}}{\partial R} \frac{\vec{\partial}}{\partial P} \\
& +\frac{1}{\hbar}\left[\mathbf{p}^{\mathrm{T}} \mathcal{V}(R) \vec{\nabla}_{\mathbf{q}}-\mathbf{q}^{\mathrm{T}} \mathcal{V}(R) \vec{\nabla}_{\mathbf{p}}\right] \\
& -\frac{\hbar}{8}\left[\mathbf{p}^{\mathrm{T}} \mathcal{V}(R) \vec{\nabla}_{\mathbf{q}}-\mathbf{q}^{\mathrm{T}} \mathcal{V}(R) \vec{\nabla}_{\mathbf{p}}\right] \frac{\overleftarrow{\partial}^{2}}{\partial R^{2}} \frac{\vec{\partial}^{2}}{\partial P^{2}} \\
& +\frac{\hbar}{8}\left[\vec{\nabla}_{\mathbf{q}}^{\mathrm{T}} \mathcal{V}(R) \vec{\nabla}_{\mathbf{q}}+\vec{\nabla}_{\mathbf{p}_{l}}^{\mathrm{T}} \mathcal{V}(R) \vec{\nabla}_{\mathbf{p}}\right] \frac{\overleftarrow{\partial}}{\partial R} \frac{\vec{\partial}}{\partial P}+\mathcal{O}\left(\hbar^{2}\right)
\end{aligned}
$$

Note that because of the approximation used in QCLE (truncating up to $\mathcal{O}\left(\hat{\Lambda}_{\mathrm{n}}\right)$, the third term in $\mathcal{L}_{\mathrm{NH}}^{[1]}$ does not show up in QCLE. Also, for the spin-boson model (with $c$ and $\Delta$ as the parameters), the state dependent potential matrix $\mathcal{V}(R)=c R \cdot \sigma_{z}+\Delta \cdot \sigma_{x}$ is purely linear, hence the third in $\mathcal{L}_{\mathrm{NH}}^{[1]}$ act on $\mathcal{V}(R)$ gives strictly zero results. For a general problem, however, both the third and the forth term should be included to fully account the terms of $\mathcal{O}(\hbar)$.

Of course, the form of the Liouvillian is not the only factor that could influence the results of $C_{A B}^{[1]}(t)$. For the population dynamics, how to approximate $[\hat{A}]_{\mathrm{W}}$ and $[\hat{B}]_{\mathrm{W}}$ will also significantly influence the accuracy of these approximated methods. ${ }^{109}$ For example, when computing $\rho_{j j}(t)=\operatorname{Tr}\left[\hat{\rho}(0) e^{\frac{i}{\hbar} \hat{H} t}|j\rangle\langle j| e^{-\frac{i}{\hbar} \hat{H} t}\right]$. The standard LSCIVR approach ${ }^{16,18}$ corresponds to

$$
\begin{aligned}
C_{A B}^{\mathrm{LSC}}(t)= & \frac{\alpha_{1}}{\mathcal{Z}} \int \mathrm{d} \mathbf{R} \int \mathrm{d} \mathbf{P} \int \mathrm{d} \mathbf{q} \int \mathrm{d} \mathbf{p} \\
& \times[|i\rangle\langle i|]_{\mathrm{W}}\left[e^{-\beta \hat{H}_{0}}\right]_{\mathrm{W}} e^{\mathcal{L}_{\mathrm{LSC}}^{[1]} \cdot t}[|j\rangle\langle j|]_{\mathrm{W}},
\end{aligned}
$$

and the standard PBME approach ${ }^{25,81}$ corresponds to

$$
\begin{aligned}
C_{A B}^{\mathrm{PBME}}(t)= & \frac{\alpha_{1}}{\mathcal{Z}} \int \mathrm{d} \mathbf{R} \int \mathrm{d} \mathbf{P} \int \mathrm{d} \mathbf{q} \int \mathrm{d} \mathbf{p} \\
& \times[|i\rangle\langle i|]_{\mathrm{W}}\left[e^{-\beta \hat{H}_{0}}\right]_{\mathrm{W}} e^{\mathcal{L}_{\mathrm{LSC}}^{[1]} \cdot t}\left[\hat{a}_{j}^{\dagger} \hat{a}_{j}\right]_{\mathrm{W}},
\end{aligned}
$$

where $|j\rangle\langle j|$ is the electronic projection operator and $\hat{a}_{j}^{\dagger} \hat{a}_{j}$ is the corresponding operator in the mapping representation. Their Wigner transforms are

$$
\begin{aligned}
& {[|j\rangle\langle j|]_{\mathrm{W}}=\frac{2^{\mathcal{K}+1}}{\hbar} e^{-\frac{1}{\hbar} \sum_{i}\left(q_{i}^{2}+p_{i}^{2}\right)}\left(q_{j}^{2}+p_{j}^{2}-\frac{\hbar}{2}\right),} \\
& {\left[\hat{a}_{j}^{\dagger} \hat{a}_{j}\right]_{\mathrm{W}}=\frac{1}{2 \hbar}\left(q_{j}^{2}+p_{j}^{2}-\hbar\right) .}
\end{aligned}
$$

The numerical comparison between these two approaches has been extensively discussed in the recent work, ${ }^{109,112,113}$ and the recent development on choosing the identity operator ${ }^{109,113,114}$ has also shown to significantly improve the population dynamics, even just using a less accurate Liouvillian $\mathcal{L}_{\mathrm{LSC}}^{[1]}$. Along the same direction, one can use the mapping action variable's Wigner transform ${ }^{115}$ to construct $[\hat{A}]_{\mathrm{W}}$ and $[\hat{B}]_{\mathrm{W}}$, and engineer various shapes of Window functions for these estimators. ${ }^{107,108,116}$ This idea has also lead to significant improvement of the population dynamics. ${ }^{33}$

Finally, if one takes the $N=1$ for the nuclear DOF and $N=2$ for the electronic mapping DOF, as well as making the truncations in Eq. 166-Eq. 167 and dropping the last term in Eq. 165, the exact Liouvillian $\mathcal{L}^{[N]}$ reduces to the partially linearized Liouvillian as follows

$$
\begin{aligned}
\mathcal{L}_{\mathrm{PL}}= & \frac{P}{M} \frac{\partial}{\partial R}-\frac{2}{\hbar}\left[V_{0}(R)+\sum_{l=1}^{2} V_{\mathrm{e}}\left(R, \mathbf{q}_{l}, \mathbf{p}_{l}\right)\right] \frac{\overleftarrow{\partial}}{\partial R} \frac{\vec{\partial}}{\partial P} \\
& +\sum_{l=1}^{2} \frac{1}{\hbar}\left[\mathbf{p}_{l}^{\mathrm{T}} \mathcal{V}(R) \vec{\nabla}_{\mathbf{q}_{l}}-\mathbf{q}_{l}^{\mathrm{T}} \mathcal{V}(R) \vec{\nabla}_{\mathbf{p}_{l}}\right],
\end{aligned}
$$

which is reminiscent of the Liouvillian used in the Forward-Backward trajectory solution (FBTS) for the $\mathrm{QCLE}^{26,27}$, and is also closely related to the equation of motion in the Partial Linearized Density Matrix (PLDM) path-integral approach. ${ }^{22,117,118}$ However, we have to cautious to draw any further connections between the correlation function $C_{\mathrm{AB}}^{[N]}(t)$ in Eq. 114 and those in FBTS and PLDM, as the latter two approaches use the coherent state representation for the mapping DOFs instead of the Wigner representation used in this work, and hence should be viewed as the Hybrid Husimi (mapping)Wigner (nuclear) representation for non-adiabatic pathintegral dynamics. In addition, the recently proposed two-oscillator mapping of $\mathrm{PBME}^{119}$ also adapts the same Liouvillian $\mathcal{L}_{\mathrm{PL}}$, even though the two copies of the mapping variables are introduced through the mapping relation of the electronic states.

\section{APPENDIX E: NUMERICAL TEST OF THE NON-ADIABATIC RPMD}

Finally, we provide preliminary numerical tests one the non-adiabatic RPMD correlation function in Eq. 86. To numerically compute it with a trajectory based approach, we rewrite it as follows

$$
\begin{aligned}
& C_{A B}^{\mathrm{NRP}}(t)=\frac{\alpha_{N} \cdot \alpha_{M}}{\mathcal{Z}_{N}^{\mathrm{RP}}} \int d \boldsymbol{P} \int d \mathcal{Q} \int d \mathbf{q} \int d \mathbf{p} \\
& \times \frac{\Gamma}{|\Gamma|} \cdot|\Gamma(\mathcal{Q}, \mathbf{q}, \mathbf{p})| \cdot e^{-\beta H_{N}^{\mathrm{RP}}(\mathcal{P}, \mathcal{Q})} A(\mathcal{Q}) \cdot e^{\mathcal{L}_{\mathrm{RP}}^{[N]} t} B(\mathcal{Q}),
\end{aligned}
$$

where the initial distribution $|\Gamma(\mathcal{Q}, \mathbf{q}, \mathbf{p})| \cdot e^{-\beta H_{N}^{\mathrm{RP}}(\mathcal{P}, \mathcal{Q})}$ is sampled by Monte-Carlo (based on a simple metropolis algorithm), the dynamics is propagated by $e^{\mathcal{L}_{\mathrm{RP}}^{[N]}}$ through a simple numerical integrator, ${ }^{55}$ and each trajectory is weighted by a complex phase $\frac{\Gamma}{|\Gamma|}$. However, we can further take advantage of the pure real estimators for $A$ and $B$, as well as the Liouvillian $L_{\mathrm{RP}}^{[N]}$, to rewrite Eq. 86 as

$$
\begin{aligned}
& C_{A B}^{\mathrm{NRP}}(t)=\frac{\alpha_{N} \cdot \alpha_{M}}{\mathcal{Z}_{N}^{\mathrm{RP}}} \int d \mathcal{P} \int d \mathcal{Q} \int d \mathbf{q} \int d \mathbf{p} \\
& \times \operatorname{sgn}(\operatorname{Re} \Gamma) \cdot|\operatorname{Re} \Gamma(\mathcal{Q}, \mathbf{q}, \mathbf{p})| \cdot e^{-\beta H_{N}^{\mathrm{RP}}(\mathcal{P}, \mathcal{Q})} A(\mathcal{Q}) \cdot e^{\mathcal{L}_{\mathrm{RP}}^{[N]} B(\mathcal{Q}),}
\end{aligned}
$$


where $\operatorname{sgn}(\operatorname{Re} \Gamma)$ is the sign (plus or minus) of the real part of $\Gamma$. The above expression is based on the fact that $C_{A B}^{[N]}(t)$ is pure real and the $\operatorname{Im} \Gamma$ part is completely separated from the $\operatorname{Re} \Gamma$, and does not contribute to the value of the $C_{A B}^{[N]}(t)$. (Note that the ensemble average of $\operatorname{Im} \Gamma$ is 0 , but $\operatorname{Im} \Gamma$ for individual trajectory is not).

We adapt a commonly used model system that contains one nuclear coordinate and two electronic states $^{54,57}$

$$
\hat{H}=\frac{\hat{P}^{2}}{2 M}+\frac{1}{2} M \omega^{2} \hat{R}^{2}+\left[\begin{array}{cc}
\varepsilon+c \hat{R} & \Delta \\
\Delta & -\varepsilon-c \hat{R}
\end{array}\right],
$$

where $\Delta$ is the electronic coupling, $c$ is the vibronic coupling, and $2 \varepsilon$ is the energy bias between the two diabatic states. We choose a reduced unit system such that $M=\hbar=1$ and $\omega=\beta=c=1$. We choose $N=8$ beads for model I, and $N=6$ beads for model II-IV. A total of $10^{5}$ trajectories are used for tight numerical convergence, even though only $10^{3}$ trajectories are good enough to provide the basic trend.

Table I presents the parameters for all of the model systems used in this paper. In particular, Model I is in the adiabatic regime, where $\Delta \gg \beta^{-1}$; Model II and III are in the non-adiabatic regime, where $\Delta \ll \beta^{-1}$; Model IV is in the intermediate regime, where $\Delta \sim \beta^{-1}$. Model III is an asymmetric case with finite diabatic energy bias $2 \epsilon$, and the rest of the model systems are symmetric cases with $\epsilon=0$.

\begin{tabular}{lllll}
\hline \hline & I & II & III & IV \\
\hline$\varepsilon$ & 0 & 0 & 1.5 & 0 \\
$\Delta$ & 10 & 0.10 & 0.10 & 1 \\
\hline \hline
\end{tabular}

TABLE I. Parameters (in a.u.) for model systems I-IV .

Fig. 1 presents the nuclear position auto-correlation function computed from NRPMD (black) and the numerical exact method (red) for Models I-IV. Model I in Fig. 1a is in the adiabatic regime. In this case, NRPMD goes back to the standard RPMD, and agrees with the exact result due to the near Harmonic adiabatic potential. Model II in Fig. 1b is in the non-adiabatic regime. This is the most challenging case and the most relevant regime for non-adiabatic electron transfer ${ }^{46}$ and proton-coupled electron transfer reactions. ${ }^{47}$ In this regime, mean field RPMD starts to break down even at a very short time, as shown in the previous work. ${ }^{57}$ NRPMD on the other hand, performs reasonably well compared to exact DVR calculations at the longer time. Models III corresponds to the asymmetric non-adiabatic regime (Fig. 1c) with diabatic energy bias $2 \epsilon$ and model IV is in the intermediate regime. In this regime, NRPMD behaves reasonably well.

We find that the numerical results obtained with the current NRPMD formalism (for the current model systems) are not significantly different than those obtained
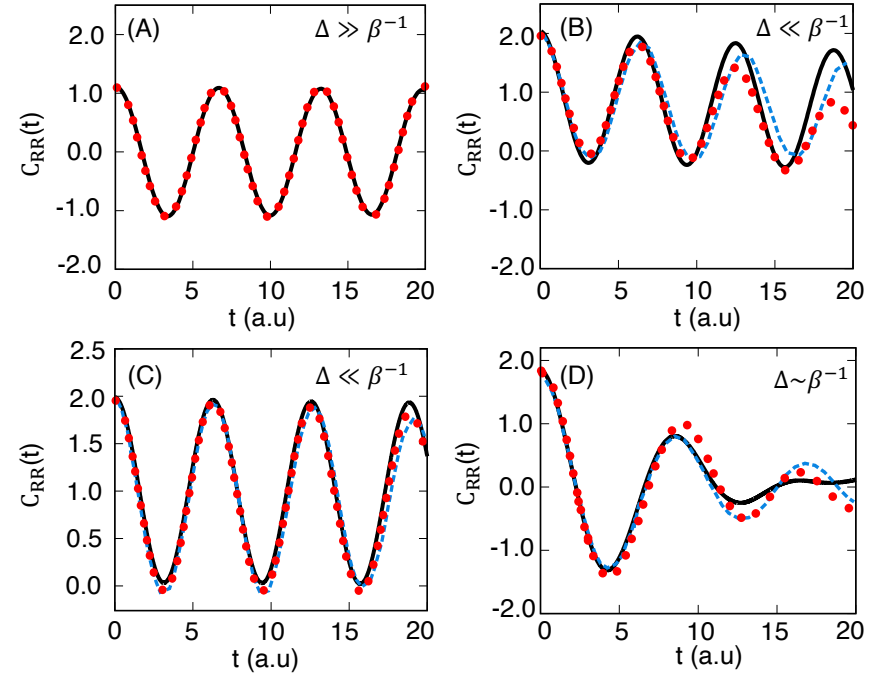

FIG. 1. The Kubo-transformed nuclear position autocorrelation function for model I-IV obtained from NRPMD (black solid), MV-RPMD (blue dashed) and numerical exact results (red dots). Results for Model I (symmetric, adiabatic) are in panel (a), Model II (symmetric, non-adiabatic) are in panel (b), Model III (asymmetric, non-adiabatic) are in panel (c), and Model IV (symmetric, intermediate) are in panel (d).

from the original NRPMD,${ }^{54}$ due to the same Liouvillian used in both formalisms. On the other hand, the correlation function obtained from MV-RPMD ${ }^{57}$ starts to oscillate with a different frequency (see Fig 1b) compared to the quantum result at a longer time, especially for model II and III, even though it uses the same initial QBD for NRPMD. This might happen because of the inter-bead couplings for mapping DOF in the Liouvillian (Eq. 92), which starts to contaminate the physical frequency of the system. The same behavior has also been found for population related quantities. ${ }^{57}$ On the other hand, MV-RPMD does preserve QBD for any arbitrary number of beads $N$, whereas for a finite number of $N$, NRPMD does not preserve QBD. ${ }^{54,55,95}$

${ }^{1}$ H. Meyer, U. Manthe, and L. Cederbaum, Chem. Phys. Lett. 165, 73 (1990).

${ }^{2}$ M. Beck, A. Jackle, G. Worth, and H. Meyer, Phys. Rep. 324, 1 (2000).

${ }^{3}$ H. Wang and M. Thoss, J. Chem. Phys 119, 1289 (2003).

${ }^{4}$ P. Eisenbrandt, M. Ruckenbauer, S. Römer, and I. Burghardt, J. Chem. Phys. 149, 174101 (2018).

${ }^{5}$ G. W. Richings and S. Habershon, J. Chem. Phys. 148, 134116 (2018).

${ }^{6}$ D. Makhov, W. Glover, T. Martinez, and D. Shalashilin, J. Chem. Phys. 141, 054110 (2014).

${ }^{7}$ B. F. E. Curchod and T. J. Martinez, Chem. Rev. 118, 3305 (2018).

${ }^{8}$ P. L. Walters and N. Makri, J. Chem. Phys. 144, 044108 (2016).

${ }^{9}$ P. L. Walters and N. Makri, J. Phys. Chem. Lett 6, 4959 (2015).

${ }^{10}$ S. M. Greene and V. S. Batista, J. Chem. Theory Comput. 13, 4034 (2017).

${ }^{11}$ J. Ren, Z. Shuai, and G. Chan, J. Chem. Theory Comput. 14, 5027 (2018).

${ }^{12}$ J. Tully, J. Chem. Phys. 93, 1061 (1990). 
${ }^{13}$ J. E. Subotnik, A. Jain, B. Landry, A. Petit, W. Ouyang, and N. Bellonzi, Annu. Rev. Phys. Chem. 67, 387 (2016).

${ }^{14}$ L. Wang, A. Akimov, and O. V. Prezhdo, J. Phys. Chem. Lett 7, 2100 (2016).

${ }^{15}$ R. Crespo-Otero and M. Barbatti, Chem. Rev. 118, 7026 (2018).

${ }^{16}$ W. H. Miller, J. Phys. Chem. A. 105, 2942 (2001).

${ }^{17}$ W.H.Miller, J. Phys. Chem. A. 113, 1405 (2009).

${ }^{18}$ X. Sun, H. Wang, and W. H. Miller, J. Chem. Phys 109 (1998).

${ }^{19}$ Q. Shi and E. Geva, J. Phys. Chem. A. 108 (2004).

${ }^{20}$ S. Bonella and D. Coker, J. Chem. Phys. 122, 194102 (2005).

${ }^{21}$ N. Makri, Phys. Chem. Chem. Phys. 13 (2011).

${ }^{22}$ P. Huo and D. F. Coker, J. Chem. Phys. 135, 201101 (2011).

${ }^{23}$ M. Lee, P. Huo, and D. F. Coker, Annu. Rev. Phys. Chem. 67, 27 (2016).

${ }^{24}$ D. M. Kernan, G. Ciccotti, and R. Kapral, J. Phys. Chem. B 112, 424 (2008)

${ }^{25}$ H. Kim, A. Nassimi, and R. Kapral, J. Chem. Phys 129, 084102 (2008).

${ }^{26}$ C. Hsieh and R. Kapral, J. Chem. Phys. 137, 22 A507 (2012).

${ }^{27}$ C. Hsieh and R. Kapral, J. Chem. Phys. 138, 134110 (2013).

${ }^{28}$ R. Kapral, J. Phys.: Condens. Mat. 27, 073201 (2015).

${ }^{29}$ P. V. Parandekar and J. C. Tully, J. Chem. Theo. Comp. 2, 229 (2006).

${ }^{30}$ J. R. Schmidt, P. V. Parandekar, and J. C. Tully, J. Chem. Phys. 129, 044104 (2008).

${ }^{31}$ S. Habershon and D. E. Manolopoulos, J. Chem. Phys. 131, 244518 (2009).

${ }^{32}$ U. Muller and G. Stock, J. Chem. Phys. 111, 77 (1999).

${ }^{33}$ W. H. Miller and S. J. Cotton, Faraday Discuss. 195, 9 (2016).

${ }^{34}$ A. Kelly, N. J. Brackbill, and T. E. Markland, J. Chem. Phys. 142, 094110 (2015).

${ }^{35}$ A. Kelly, A. Montoya-Castillo, L. Wang, and T. E. Markland, J. Chem. Phys. 144, 184105 (2016).

${ }^{36}$ E. Mulvihill, A. Schubert, X. Sun, B. D. Dunietz, and E. Geva, J. Chem. Phys. 150, 034101 (2019).

${ }^{37}$ B. J. Berne and D. Thirumalai, Annu. Rev. Phys. Chem. 37, 401 (1986).

${ }^{38}$ D. M. Ceperley, Rev. Mod. Phys. 67, 279 (1995).

${ }^{39}$ D. Chandler and P. G. Wolynes, J. Chem. Phys. 74, 4078 (1981).

${ }^{40}$ J. Cao and G. Voth, J. Chem. Phys. 100, 5106 (1994).

${ }^{41}$ Jang and G. Voth, J. Chem. Phys. 111, 2371 (1999).

${ }^{42}$ Q. Shi and E. Geva, J. Chem. Phys. 118 (2003).

${ }^{43}$ S. Habershon, D. E. Manolopoulos, T. E. Markland, and T. F. Miller, Annu. Rev. Phys. Chem. 64, 124105 (2013).

${ }^{44}$ I. R. Craig and D. E. Manolopoulos, J. Chem. Phys. 121, 3368 (2004).

${ }^{45}$ A. R. Menzeleev, N. Ananth, and T. F. Miller, J. Chem. Phys. 132, 034106 (2010)

${ }^{46}$ A. R. Menzeleev, N. Ananth, and T. F. Miller, J. Chem. Phys. 135, 074106 (2011)

${ }^{47}$ J. S. Kretchmer and T. F. Miller, J. Chem. Phys. 138, 134109 (2013).

${ }^{48}$ J. Kretchmer and T. F. Miller, Inorg. Chem. 55, 1022 (2016).

${ }^{49}$ R. L. Kenion and N. Ananth, hys. Chem. Chem. Phys. 18, 26117 (2016).

${ }^{50}$ R. F. N. Boekelheide and T. F. Miller, Proc. Natl. Acad. Sci. USA 108, 16159 (2011).

${ }^{51}$ S. L.Wang, S.D.Fried and T. E. Markland, Proc. Natl. Acad. Sci. USA 111, 18454 (2014).

${ }^{52}$ O. Marsalek and T. E. Markland, J. Phys. Chem. Lett. 8, 1545 (2017).

${ }^{53}$ T. E. Markland and M. Ceriotti, Nature Rev. Chem. 2, 1 (2018).

${ }^{54}$ J. O. Richardson and M. Thoss, J. Chem. Phys. 139, 031102 (2013).

55 J. O. Richardson, P.Meyer, M.-O.Pleinert, and M.Thoss, Chemical Physics 482, 124 (2017).

${ }^{56}$ S.N.Chowdhury and P. Huo, J. Chem. Phys 150, 244102 (2019).

${ }^{57}$ N. Ananth, J. Chem. Phys. 139, 124102 (2013).

${ }^{58}$ J.R.Duke and N. Ananth, J. Phys. Chem. Lett. 06, 4219 (2015).

${ }^{59}$ S. Pierre, J. R. Duke, T. J. H. Hele, and N. Ananth, J. Chem.
Phys. 147, 234103 (2017).

${ }^{60}$ T. Yoshikawa and T. Takayanagi, Chem. Phys. Letts. 564, 1 (2013).

${ }^{61}$ A. R. Menzeleev, F. Bell, and T. F. Miller, J. Chem. Phys. 140, 064103 (2014).

${ }^{62}$ J. Kretchmer, N. Boekelheide, J. F. Warren, J. R. Winkler, H. B. Gray, and T. F. Miller, Proc. Natl. Acad. Sci. USA 115, 6129 (2018).

${ }^{63}$ S.N.Chowdhury and P. Huo, J. Chem. Phys 147, 214109 (2017).

${ }^{64}$ P. Shushkov, R. Li, and J. C. Tully, J. Chem. Phys. 137, 22 A549 (2012).

${ }^{65}$ F. A. Shakib and P. Huo, J. Phys. Chem. Lett. 8, 3073 (2017).

${ }^{66}$ X. Tao, P. Shushkov, and T. F. Miller, J. Chem. Phys. 148, 102327 (2018).

${ }^{67}$ X. Tao, P. Shushkov, and T. F. Miller, J. Phys. Chem. A. 123, 3013 (2019).

${ }^{68}$ R. Kaur and R. Welsch, J. Chem. Phys. 150, 114105 (2019).

${ }^{69}$ T. J. H. Hele, M. J. Willatt, A. Muolo, and S. C. Althorpe, J. Chem. Phys. 142, 134103 (2015)

${ }^{70}$ M.J.Willatt, Ph.D. Thesis: Matsubara Dynamics and its Practical Implementations (2017).

${ }^{71}$ K. A. Jung, P. E. Videla, and V. S. Batista, The Journal of Chemical Physics 151, 034108 (2019).

${ }^{72}$ K. A. Jung, P. E. Videla, and V. S. Batista, The Journal of Chemical Physics 151, 034108 (2019).

${ }^{73}$ T. J. H. Hele, M. J. Willatt, A. Muolo, and S. C. Althorpe, J. Chem. Phys. 142, 191101 (2015).

${ }^{74}$ H. D. Meyer and W. H. Miller, J. Chem. Phys 70, 3214 (1979).

${ }^{75}$ G. Stock and M. Thoss, Phys. Rev. Lett. 78, 578 (1997).

${ }^{76}$ G. Stock and M. Thoss, Phys. Rev. A. 59, 64 (1999).

${ }^{77}$ N. Ananth and T. F. Miller, J. Chem. Phys 133, 234103 (2010).

${ }^{78}$ T. J. H. Hele and N. Ananth, Faraday Discuss. 195, 269 (2016).

${ }^{79}$ M. Hillery, R. O'Connell, M. Scully, and E. Wigner, Phys. Rep. 106, 121 (1984)

${ }^{80}$ J.E.Moyal, Mathematical Proceedings of the Cambridge Philosophical Society 45, 99124 (1949).

${ }^{81}$ A. N. S. Bonella and R. Kapral, J. Chem. Phys. 133, 134115 (2010).

${ }^{82}$ P. Eherenfest, Zeitschrift für Physik" 45, 455 (1927).

${ }^{83}$ H. W. Kim and Y. M. Rhee, J. Chem. Phys. 140, 184106 (2014).

${ }^{84} \mathrm{H}$. Groenewold, Physica 12, 405 (1946).

${ }^{85}$ M.Ceriotti, M. Parrinello, T. E. Markland, and D. E. Manolopoulos, J. Chem. Phys 133, 124104 (2010).

${ }^{86}$ G. Trenins and S. C. Althorpe, J. Chem. Phys. 149, 014102 (2018).

${ }^{87}$ G. Trenins and M. J. W. S. C. Althorpe, J. Chem. Phys. 151, 054109 (2019).

${ }^{88}$ M. J. Willatt, M. Ceriotti, and S. C. Althorpe, J. Chem. Phys. 148, 102336 (2018).

${ }^{89}$ C. Chakravarty, Int. Rev. Phys. Chem. 16, 421 (1997)

${ }^{90}$ C. Chakravarty, M. C. Gordillo, and D. M. Ceperley, J. Chem. Phys. 109, 2123 (1998).

${ }^{91}$ D. L. Freeman and J. D. Doll, J. Chem. Phys. 80, 11 (1984).

${ }^{92}$ C. M. Bender, D. C. Brody, and D. W. Hook, J. Phys. A Math. Theor 41, 352003 (2008).

${ }^{93}$ M.Rossi, M.Ceriotti, and D. E. Manolopoulos, J. Chem. Phys 140, 234116 (2014)

${ }^{94}$ T. Hele, Mol. Phys. 115, 1435 (2017).

${ }^{95}$ M. A. C. Saller, J. E. Runeson, and J. O. Richardson, Quantum Chemistry and Dynamics of Excited States: Methods and Applications (John Wiley Sons Ltd), 629 (2020).

${ }^{96}$ R.Welsch, K.Song, Q.Shi, S. C. Althorpe, and T. F. Miller, J. Chem. Phys 145, 204118 (2016).

${ }^{97}$ P. S. R. P. Steele, J. Zwickl and J. C. Tully, J. Chem. Phys. 134, 074112 (2011).

${ }^{98}$ J.-L. Liao and G. A. Voth, J. Phys. Chem. B. 106, 8449 (2002).

${ }^{99}$ J. E. Subotnik, W. Ouyang, and B. R. Landry, J. Chem. Phys. 139, 214107 (2013).

${ }^{100}$ R. Kapral, Chem. Phys. 481, 77 (2016).

${ }^{101}$ S. C. Althorpe, N. Ananth, G. Angulo, R. D. Astumian, V. Beni- 
wal, J. Blumberger, P. G. Bolhuis, B. Ensing, D. R. Glowacki, S. Habershon, S. Hammes-Schiffer, T. J. H. Hele, N. Makri, D. E. Manolopoulos, L. K. McKemmish, T. F. M. III, W. H. Miller, A. J. M. andTatiana Nekipelova, E. Pollak, J. O. Richardson, M. Richter, P. R. Chowdhury, D. Shalashilin, and R. Szabla, Faraday Discuss 195, 311 (2016).

102 J. E. Runeson and J. O. Richardson, J. Chem. phys. 151, 044119 (2019).

103 J. E. Runeson and J. O. Richardson, J. Chem. phys. 152, 084110 (2020).

${ }^{104}$ J. R. Mannoucha and J. O. Richardson, J. Chem. phys. 153, 194109 (2020)

${ }^{105}$ J. R. Mannoucha and J. O. Richardson, J. Chem. phys. 153, 194110 (2020).

${ }^{106}$ J. Liu, J. Chem. Phys. 145, 204105 (2016).

${ }^{107}$ S. J. Cotton and W. H. Miller, J. Chem. Phys. 150, 104101 (2019).

${ }^{108}$ S. J. Cotton and W. H. Miller, J. Chem. Phys. 139, 234112
(2013).

${ }^{109}$ M. A. C. Saller, A. Kelly, and J. O. Richardson, J. Chem. Phys 150, 071101 (2019).

${ }^{110}$ W. B. Case, Am. J. Phys. 76, 937 (2008).

${ }^{111}$ R. Kapral, Ann. Rev. Phys. Chem. 57, 129 (2006).

${ }^{112}$ E. Mulvihill, X. Gao, Y. Liu, A. Schubert, B. D. Dunietz, and E. Geva, J. Chem. Phys. 151, 074103 (2019).

${ }^{113}$ X. Gao, M. A. C. Saller, Y. Liu, A. Kelly, J. O. Richardson, and E. Geva, J. Chem. Theory Comput. 16, 28832895 (2020).

${ }^{114}$ M. A. C. Saller, A. Kelly, and J. O. Richardson, Faraday Discuss. 221, 150167 (2020).

${ }^{115}$ W. H. Miller and S.J.Cotton, J. Chem. Phys. 145, 081102 (2016).

${ }^{116}$ S. J. Cotton and W. H. Miller, J. Chem. Phys. 145, 144108 (2016).

${ }^{117}$ P. Huo and D. F. Coker, Mol. Phys. 110, 1035 (2012).

${ }^{118}$ P. Huo and D. F. Coker, J. Chem. Phys. 137, 22A535 (2012).

${ }^{119}$ H. W. Kim and Y. M. Rhee, J. Chem. Phys. 153, 214103 (2020). 